\title{
High-precision percolation thresholds and Potts-model critical manifolds from graph polynomials
}

\author{
Jesper Lykke Jacobsen ${ }^{1,2}$ \\ ${ }^{1}$ LPTENS, École Normale Supérieure, 24 rue Lhomond, 75231 Paris, France \\ ${ }^{2}$ Université Pierre et Marie Curie, 4 place Jussieu, 75252 Paris, France \\ E-mail: jesper.jacobsen@ens.fr
}

\begin{abstract}
.
The critical curves of the $q$-state Potts model can be determined exactly for regular two-dimensional lattices $G$ that are of the three-terminal type. This comprises the square, triangular, hexagonal and bowtie lattices. Jacobsen and Scullard have defined a graph polynomial $P_{B}(q, v)$ that gives access to the critical manifold for general lattices. It depends on a finite repeating part of the lattice, called the basis $B$, and its real roots in the temperature variable $v=\mathrm{e}^{K}-1$ provide increasingly accurate approximations to the critical manifolds upon increasing the size of $B$. Using transfer matrix techniques, these authors computed $P_{B}(q, v)$ for large bases (up to 243 edges), obtaining determinations of the ferromagnetic critical point $v_{\mathrm{c}}>0$ for the $\left(4,8^{2}\right)$, kagome, and $\left(3,12^{2}\right)$ lattices to a precision (of the order $10^{-8}$ ) slightly superior to that of the best available Monte Carlo simulations.

In this paper we describe a more efficient transfer matrix approach to the computation of $P_{B}(q, v)$ that relies on a formulation within the periodic TemperleyLieb algebra. This makes possible computations for substantially larger bases (up to 882 edges), and the precision on $v_{\mathrm{c}}$ is hence taken to the range $10^{-13}$. We further show that a large variety of regular lattices can be cast in a form suitable for this approach. This includes all Archimedean lattices, their duals and their medials. For all these lattices we tabulate high-precision estimates of the bond percolation thresholds $p_{\mathrm{c}}$ and Potts critical points $v_{\mathrm{c}}$. We also trace and discuss the full Potts critical manifold in the $(q, v)$ plane, paying special attention to the antiferromagnetic region $v<0$. Finally, we adapt the technique to site percolation as well, and compute the polynomials $P_{B}(p)$ for certain Archimedean and dual lattices (those having only cubic and quartic vertices), using very large bases (up to 243 vertices). This produces the site percolation thresholds $p_{\mathrm{c}}$ to a precision of the order $10^{-9}$.
\end{abstract}

\section{Introduction}

The notion of exact solvability plays a prominent role within the field of two-dimensional statistical physics. The exact solutions of a certain number of lattice models - such as the Ising model [1], dimer coverings [2], the six-vertex [3] and eight-vertex models [4], 
and the Potts model [5] - have served as milestones by which the advance of the field can be judged, and as benchmarks for analytical and numerical methods.

In this context, the question of what makes a non-trivial model exactly solvable is obviously of high importance. One may ask what role does the choice of lattice play in the solvability. All models cited were initially solved on the simplest possible, square lattice. It quickly turned out that the solutions of the Ising and dimer models could be extended to essentially any regular two-dimensional lattice, whereas vertex and Potts models have only been solved on a few other simple lattices. It is of interest to solve these models, or find accurate approximate solutions, on more general lattices, such as the remaining Archimedean lattices.

We here examine this issue in the context of the $q$-state Potts model [6]. Given a connected graph $G=(V, E)$ with vertex set $V$ and edge set $E$, its partition function $Z$ is can be defined as [7]

$$
Z=\sum_{A \subseteq E} v^{|A|} q^{k(A)},
$$

where $|A|$ denotes the number of edges in the subset $A$, and $k(A)$ is the number of connected components (including isolated vertices) in the subgraph $G_{A}=(V, A)$. The temperature variable is denoted $v=\mathrm{e}^{K}-1$, where $K$ is the reduced interaction energy between adjacent $q$-component spins. In the representation (1) we shall formally allow both $q$ and $v$ to take arbitrary real values.

A first aspect to be addressed when solving the Potts model defined on some lattice $G$ is the determination of the values, for any given $q$, of the temperature $v$ where a phase transition takes place. We shall not be concerned here with the nature (order) of phase transitions, and simply refer to the set of transition temperatures in the real $(q, v)$ plane as the critical manifold. The critical manifold has only been determined exactly when $G$ is the square [5], triangular [8], hexagonal (the dual of the former), and bowtie [9] lattices, as well as certain decorations of these lattices [10]. More precisely, the solvable lattices are all of the three-terminal type, that is, they are regular arrangements of triangles, each consisting of three boundary spins (or terminals) and an arbitrary number of internal spins. The interactions inside each triangle can take any form, but distinct triangles only interact through the terminals. The triangles can be disposed as the up-pointing triangles in a triangular lattice [11], or in a bowtie pattern [9].

By contrast, lattices of the four-terminal type do not appear to be exactly solvable. $\mathrm{Wu}$ has however shown that in a number of cases their critical manifolds can be well approximated by a homogeneity assumption [12]. Very recently, Jacobsen and Scullard have defined a graph polynomial $P_{B}(q, v)$ that depends on a finite repeating part of the lattice, called the basis $B$, and reduces to Wu's expressions for the smallest possible choices of $B$ [13]. The real roots of $P_{B}(q, v)$ in the temperature variable $v=\mathrm{e}^{K}-1$ provide increasingly accurate approximations to the critical manifolds upon increasing the size of $B$. Using transfer matrix techniques, these authors computed $P_{B}(q, v)$ for large bases (up to 243 edges), obtaining determinations of the ferromagnetic critical 
point $v_{\mathrm{c}}>0$ for the four-eight, kagome, and three-twelve lattices to a precision of the order $10^{-8}$ [14], slightly superior to that of the best available Monte Carlo simulations.

For $q=1$ the polynomial $P_{B}(q, v)$ reduces to the bond percolation polynomial introduced by Scullard and Ziff $[15,16,17]$ and studied further in $[18,19,20,21]$.

The goal of this paper is twofold. First, we extend the set of lattices that can be studied to all Archimedean lattices, their dual (Laves) lattices, as well as their medial (or surrounding) lattices. $\dagger$ Second, we describe a transfer matrix approach to the computation of $P_{B}(q, v)$ that is more efficient than the one given in Ref. [14]. On a technical level, this is done by representing all these lattices in a particular four-terminal form, and writing the $\check{R}$-matrix of the fundamental building blocks in terms of operators acting within the periodic Temperley-Lieb algebra. From a practical point of view, this makes possible computations for substantially larger bases (up to 882 edges) than those used in Ref. [14]. The precision on $v_{\mathrm{c}}$ is hence taken to the range $10^{-13}$, far ahead of any competing perturbative or numerical technique.

We further compute graph polynomials $P_{B}(p)$ for site percolation problems on several different lattices. $\neq$ For practical reasons, we limit ourselves in this case to Archimedean and dual lattices having only cubic and quartic vertices (i.e., no vertex has degree $\geq 5$ ) - but it will become clear that this is not an essential limitation of the method. It turns out that the estimates for the percolation threshold $p_{\mathrm{c}}$ do not converge as fast as in the case of bond percolation, or for $v_{\mathrm{c}}$ in the Potts model. Accordingly we obtain $p_{\mathrm{c}}$ to a precision which is typically of the order $10^{-8}$, and sometimes even $10^{-9}$. This precision is however still superior to that of the best simulation results.

For the exactly solvable lattices, $P_{B}(q, v)$ was found to factorise in a number of cases $[13,14]$, shedding a small factor that corresponds to the exactly known critical curve(s). But it was also observed $[13,14]$ that the remaining, large factor contains pertinent information about the phase diagram in the region $v<0$. We continue these investigations here, by tracing the full Potts critical manifold in the $(q, v)$ plane, using the larger basis and the substantially larger selection of lattices now at hand.

Throughout the paper the estimates for $v_{\mathrm{c}}$ coming from finite bases $B$ and their extrapolation to the thermodynamic limit will be presented, for each lattice, in table form for easy perusal. However, to give the reader a very concrete idea about the precision attained by the present method, we now briefly present a few sample results.

- For the bond percolation threshold on the three-twelve lattice our graph polynomial result and the currently best available numerical calculation (diagonalisation of the transfer matrix [22]) read respectively

$$
p_{\mathrm{c}}= \begin{cases}0.7404207988474(7) & \text { (Graph polynomial, this work) } \\ 0.740420800(2) & \text { (Graph polynomial, Ref. [21]) } \\ 0.74042077(2) & \text { (Transfer matrices) }\end{cases}
$$

$\dagger$ The Archimedean lattices were previously considered in the special case $q=1$ by Scullard [19, 20], using bases of size up to 36 edges. These bases include our $n=2$ square bases for the kagome [19], four-eight, three-twelve, snub square, snub hexagonal and ruby lattices [20].

$\ddagger$ This generalises the earlier work [21] to several new lattices and to considerably larger bases. 
where the number in parentheses is the error bar on the last given digit. We have also shown for comparison the best graph polynomial result [21] prior to the improvements presented in this paper.

- For the Ising model, $q=2$, it was previously observed $[13,14]$ that $P_{B}(q, v)$ invariable factorises. Our results are thus exact in that case. For the Archimedean lattices and their duals, all our results coincide with those obtained in Ref. [23] from the Feynman-Vdovichenko combinatorial approach. The Ising case therefore strongly supports the correctness of the method for those lattices. However, for some of the medial lattices our Ising results are new (although we believe they could easily be derived, e.g., using the methods of [23]).

- For the $q=3$ state Potts model on the kagome lattice we can compare with the best available series estimate (67-term low-temperature series [24]):

$$
v_{\mathrm{c}}= \begin{cases}1.8764595742(1) & \text { (Graph polynomial) } \\ 1.87646(5) & \text { (Series expansion) }\end{cases}
$$

Our precision for $q=4$ is similar and notably does not suffer from the logarithmic corrections usually associated with the presence of a marginally irrelevant operator.

- For the site percolation threshold on the square lattice, the graph polynomial has a more modest performance, but the precision is still better than that of the best available numerical calculation (Monte Carlo simulation [25]):

$$
p_{\mathrm{c}}= \begin{cases}0.59274601(2) & (\text { Graph polynomial }) \\ 0.59274605(3) & \text { (Monte Carlo) }\end{cases}
$$

The organisation of the paper is as follows. In section 2 we present the lattices to be studied and introduce some useful terminology. The technical centrepiece of this work is section 3, where we discuss how the graph polynomial $P_{B}(q, v)$ can be expressed in terms of the periodic Temperley-Lieb algebra. Some readers might want to skip that section at a first reading and go straight to the results. Those are presented in section 4 for the Potts model and bond percolation on the Archimedean lattices, and in sections 5-6 for the same models on the dual and medial lattices. In section 7 we explain how to compute the graph polynomial $P_{B}(p)$ for site percolation and we give results for the Archimedean and dual lattices with only cubic and quartic vertices. Finally, section 8 contains the discussion and some concluding remarks.

\section{Archimedean lattices, their duals, and their medials}

The eleven Archimedean lattices are shown in Figure 1. By definition, an Archimedean lattice is such that each vertex is surrounded by the same types of faces, appearing in the same cyclic order. For instance, each vertex of the lattice shown in Figure 1(k) is surrounded by a triangle, a square, a hexagon, and another square, so this lattice is called $(3,4,6,4)$ in the notation of Grünbaum and Shephard [26]. The corresponding dual lattice is denoted $D(3,4,6,4)$. By definition, the dual lattice is obtained from 


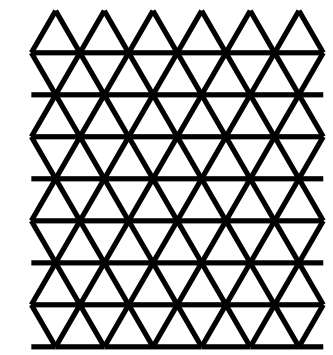

(a)

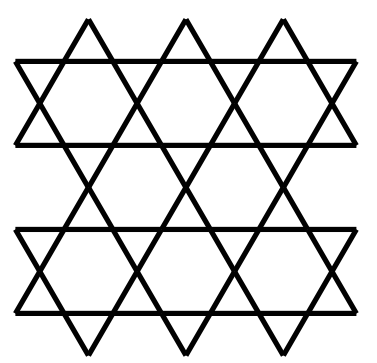

(d)

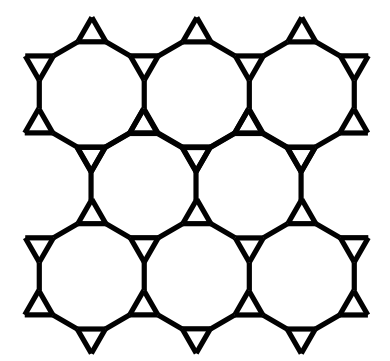

(g)

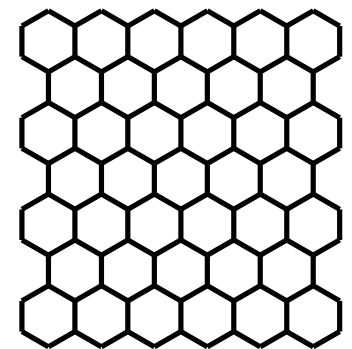

(b)

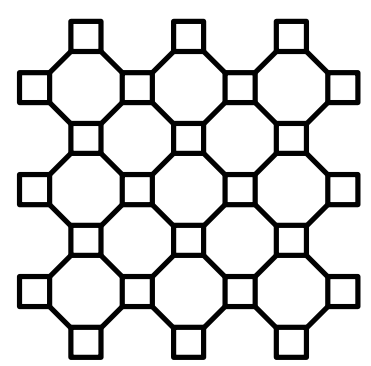

(e)

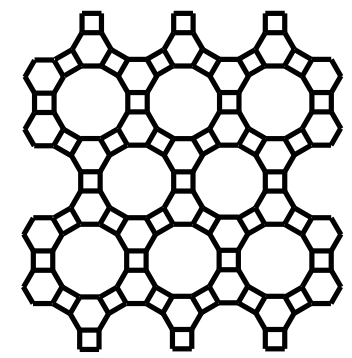

(h)

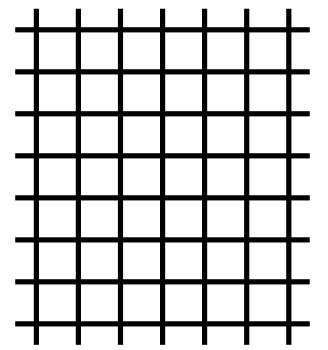

(c)

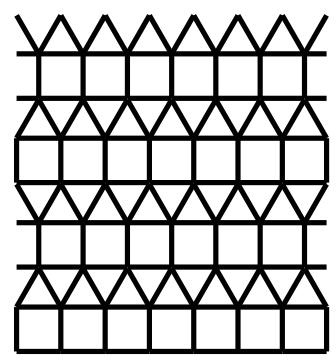

(f)

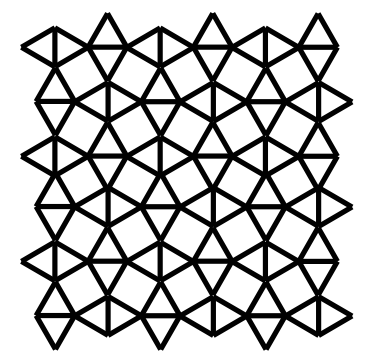

(i)

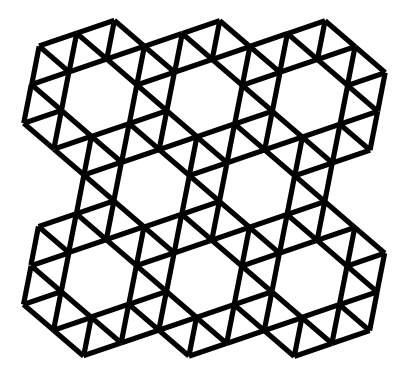

(j)

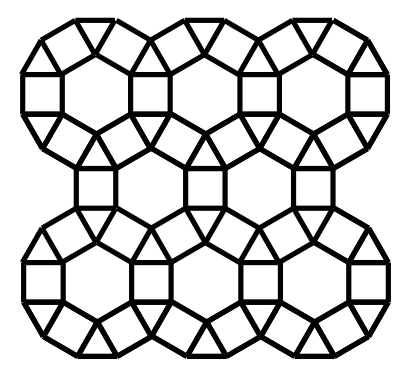

(k)

Figure 1. The eleven Archimedean lattices. Their names are given in Table 1.

the primal one by replacing vertices by faces, and vice versa, and by replacing edges by intersecting dual edges. It follows in particular that an Archimedean dual consists of identical faces (or tiles) and that the vertices bordering each tile have the degrees specified by the labels. So $D(3,4,6,4)$ is a quadrangulation (since there are four labels), and each quadrangle is bordered by vertices of degrees $3,4,6$, and 4 .

The Archimedean lattices and their duals have convenient nicknames, shown in Table 1, that we shall often use throughout this work. For instance, $(3,4,6,4)$ is known 
as the ruby lattice.

Note that the square lattice is selfdual, while the triangular and hexagonal lattices are mutually dual. In other words, $D\left(4^{4}\right)=\left(4^{4}\right)$ and $D\left(3^{6}\right)=\left(6^{3}\right)$. These three are the only three-terminal (hence exactly solvable) lattices. We show in this paper that the remaining eight lattices are of the four-terminal type, as required by our specific transfer matrix setup.

The graph polynomial for the Potts model on the dual lattice is obtained from its primal counterpart $P_{B}(q, v)$ by replacing $v$ by $v^{*}=q / v$, and multiplying with an (unimportant) overall factor. This connexion is identical to the well-known duality relation $[6,27]$ that relates the partition function of the Potts model on the primal and dual lattices. For site percolation we do not have such a duality relation. Moreover, the notion of three-terminal and four-terminal lattices changes slightly for site percolation, since the terminals are now midpoints of edges; this will be discussed in section 7 .

For the Potts model we also consider the medial lattices, obtained from the primal lattices by placing vertices on the midpoints of edges, and placing medial edges cyclically around the primal faces [28]. Medial lattices are also known as surrounding lattices. It follows that the medial lattice has faces corresponding to each of the primal faces, and to each of the primal vertices, with the same degree. Moreover, a pair of mutually dual lattices have the same medial. We denote medials by the letter $\mathcal{M}$ so that, for example, the medial of the ruby lattice is denoted $\mathcal{M}(3,4,6,4)$.

The medial lattice $\mathcal{M}(G)$ should not be confused with the covering lattice $\mathcal{C}(G)$ (also called line graph). Site percolation on $\mathcal{C}(G)$ is equivalent to bond percolation on $G[29,30]$. When the primal lattice $G$ is planar, $\mathcal{M}(G)$ is also planar; but $\mathcal{C}(G)$ will in general be non-planar, except if $G$ is a cubic lattice. $\dagger$ We have $\mathcal{M}(G)=\mathcal{M}(D(G)$ ), but the same property does not hold for covering lattices, unless $G$ is selfdual. In this paper we only deal with planar lattices, and we shall not consider covering lattices any further. Also, we shall not consider site percolation problems on medial lattices, although these are independent of bond percolation problems (except when $G$ is cubic).

Note that the medial of the square lattice is itself a square lattice, $\mathcal{M}\left(4^{4}\right)=\left(4^{4}\right)$. The medial of the triangular (and of its dual, hexagonal) lattice is the kagome lattice, itself an Archimedean lattice. In other words, $\mathcal{M}\left(3^{6}\right)=\mathcal{M}\left(6^{3}\right)=(3,6,3,6)$. The medial of the kagome lattice is the ruby lattice, $\mathcal{M}(3,6,3,6)=(3,4,6,4)$. The remaining seven medial lattices are not Archimedean. Most of them are so-called two-uniform lattices, i.e., they contain two different classes of vertices with distinct face environments, while others yet are three-uniform.

$\dagger$ For example, if $G$ is the hexagonal lattice, $\mathcal{C}(G)=\mathcal{M}(G)$ is the kagome lattice. Site percolation on the kagome lattice is thus equivalent to bond percolation on the hexagonal lattice; both problems turn out to be exactly solvable. 


\begin{tabular}{l|ll|ll} 
& Lattice & Notation & Dual lattice & Notation \\
\hline (a) & Triangular & $\left(3^{6}\right)$ & Hexagonal & $\left(6^{3}\right)$ \\
(b) & Hexagonal & $\left(6^{3}\right)$ & Triangular & $\left(3^{6}\right)$ \\
(c) & Square & $\left(4^{4}\right)$ & Square & $\left(4^{4}\right)$ \\
(d) & Kagome & $(3,6,3,6)$ & Dice & $D(3,6,3,6)$ \\
(e) & Four-eight & $\left(4,8^{2}\right)$ & Union-jack & $D\left(4,8^{2}\right)$ \\
(f) & Frieze & $\left(3^{3}, 4^{2}\right)$ & Frieze dual & $D\left(3^{3}, 4^{2}\right)$ \\
(g) & Three-twelve & $\left(3,12^{2}\right)$ & Asanoha & $D\left(3,12^{2}\right)$ \\
(h) & Cross & $(4,6,12)$ & Bisected hexagonal & $D(4,6,12)$ \\
(i) & Snub square & $\left(3^{2}, 4,3,4\right)$ & Cairo pentagonal & $D\left(3^{2}, 4,3,4\right)$ \\
(j) & Snub hexagonal & $\left(3^{4}, 6\right)$ & Daisy & $D\left(3^{4}, 6\right)$ \\
(k) & Ruby & $(3,4,6,4)$ & Ruby dual & $D(3,4,6,4)$
\end{tabular}

Table 1. Nomenclature of the Archimedean lattices and their duals (Laves lattices). The labels (a)-(k) refer to Figure 1. The notation is that of Grünbaum and Shephard [26].

\section{Graph polynomial and the Temperley-Lieb algebra}

In this section we shall only be concerned with the Potts model (1) and the special case of bond percolation, which is obtained by setting $q=1$ and choosing the probability of an open bond as $p=\frac{v}{1+v}$. Site percolation requires a few modifications of the general setup and will be discussed in section 7 .

\subsection{Bases and embeddings}

The graph polynomial $P_{B}(q, v)$ for the Potts model depends on a finite part of the lattice, called the basis $B$, that generates the infinite lattice $G$ by an appropriate set of translations, called the embedding [14]. Each regular lattice $G$ admits an infinite number of choices for $B$. We shall be interested in the simplest possible family of $B$, called square bases in Ref. [14]. They have a checkerboard structure, shown in Figure 2, consisting of alternating grey and white squares.

The grey squares in Figure 2 can contain any arrangement of lattice edges and internal vertices. Two adjacent grey squares meet in a common vertex; we shall call such shared vertices the terminals of the grey square. The lattices $G$ which can be represented as in Figure 2 are called four-terminal lattices. The Potts model on such $G$ is not in general exactly solvable, as discussed in the Introduction. For the simplest lattices, all the grey squares contain the same arrangement of edges and internal vertices. For more complicated lattices it is necessary to let the structure of the grey squares depend on the coordinates $(x, y)$ with some periodicity.

A rectangular (resp. square) basis is an array of $n \times m$ (resp. $n \times n$ ) grey squares. We shall almost exclusively be interested in square bases. However, for a few lattices we shall need to decorate the grey squares with a periodicity that is different in the $x$ 


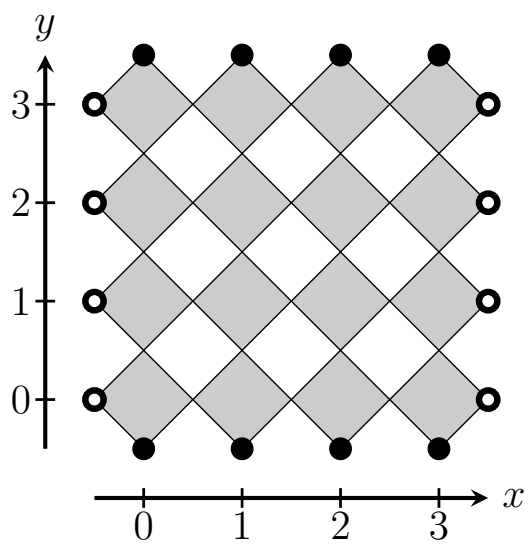

Figure 2. Square basis of size $n \times n$ with $n=4$. The horizontal (resp. vertical) terminals of the basis are shown as white (resp. black) circles. The grey squares on the checkerboard are identified by their $(x, y)$ coordinates and can contain any arrangement of lattice edges. The white square are either empty, or may contain a diagonal horizontal edge.

and $y$ directions, and in those cases we might need rectangular bases in order to respect that periodicity. The transfer matrix construction to be described below also allows for decorating (some of) the white squares by a single horizontal edge. For simplicity, we shall still say that the corresponding lattices are of the four-terminal type.

The accuracy of the critical manifold determined by $P_{B}(q, v)=0$ increases rapidly with $n$, but unfortunately the same is true for the computational effort to compute $P_{B}(q, v)$. To be precise, the contraction-deletion algorithm described in [13] has time and memory requirements that grow exponentially in $n m$, whereas for the transfer matrix algorithm of [14] the growth is only exponential in $\min (n, m)$. The improved transfer matrix algorithm to be described here is again exponential in $\min (n, m)$ but with a smaller growth constant, enabling us to access larger sizes.

The algorithm of [13] was capable of computing $P_{B}(q, v)$ for basis with up to 36 edges. Since the most interesting lattices have typically at least six edges per grey square, this means that bases of size $2 \times 2$ or $2 \times 3$ could be handled. The limit of feasibility using the transfer matrix of [14] was improved to $\min (n, m)=4$. In this work we further improve the transfer matrix algorithm, putting $n \times n$ square bases with $n=7$ within reach. To be precise, we compute exactly the two-variable Potts polynomial $P_{B}(q, v)$ up to $n=5$; the exact one-variable bond percolation polynomial $P_{B}\left(q=1, v=\frac{p}{1-p}\right)$ up to $n=6$; and roots in $v$ to 50-digit numerical precision of the equation $P_{B}(q, v)=0$ for selected values of $q$ up to $n=7$.

The site percolation polynomials $P_{B}(p)$ can sometimes be computed for even larger $n$, namely up to $n=11$ for the square lattice, and even $n=16$ for the ruby lattice (see section 7). For the site percolation problems we invariably compute the exact polynomial $P_{B}(p)$, refraining from any additional gain that might have been obtained by finding only the relevant root $\left(0<p_{\mathrm{c}}<1\right)$ with finite numerical precision. 
The vertices situated at a corner of a grey square in Figure 2, and not shared between two distinct grey squares, are called the terminals of the basis $B$. The embedding of $B$ into $G$ is defined by gluing distinct copies of $B$ at the terminals. $\dagger$ This can be done in a variety of ways, but in this work we are only interested in the simplest possibility, called straight embedding in Ref. [14]. It consists of simply translating the $n \times m$ basis horizontally through multiples of $n \mathbf{e}_{x}$, and vertically through multiples of $m \mathbf{e}_{y}$, where $\mathbf{e}_{x}$ (resp. $\mathbf{e}_{y}$ ) is a unit vector in the $x$-direction (resp. $y$-direction).

\subsection{Graph polynomial}

The graph polynomial $P_{B}(q, v)$ was initially defined from a deletion-contraction principle [13]. It was subsequently shown [14] that it can be equivalently written as a linear combination of conditioned partition functions, similar to (1), defined on a graph which is equal to the basis $B$. Consider first the partition function $Z$ of the Potts model defined on $B=(V, E)$, where we have imposed toroidal boundary conditions, i.e., opposite terminals are identified both horizontally and vertically. We can decompose

$$
Z=Z_{2 \mathrm{D}}+Z_{1 \mathrm{D}}+Z_{0 \mathrm{D}}
$$

where $Z_{0 \mathrm{D}}$ is the sum over edge subsets $A \subseteq E$ such that all connected components (clusters) in the subgraph $G_{A}=(V, A)$ have trivial homotopy on the torus (i.e., are contractible to a point), and $Z_{1 \mathrm{D}}$ is the sum over terms where there exists both a cluster and a dual cluster with non-trivial homotopy. In simpler words, $Z_{2 \mathrm{D}}$ regroups the terms where there is a cluster that spans both spatial directions; the terms in $Z_{1 \mathrm{D}}$ contain a cluster that spans only one, but not both, of the directions in space; and in $Z_{0 \mathrm{D}}$ there are no spanning clusters.

In this setup the graph polynomial reads [14]

$$
P_{B}(q, v)=Z_{2 \mathrm{D}}-q Z_{0 \mathrm{D}} .
$$

The term in $Z$ corresponding to each $A \subseteq E$ can be assigned to either $Z_{2 \mathrm{D}}, Z_{1 \mathrm{D}}$ or $Z_{0 \mathrm{D}}$ by using the Euler relation, as explained in [14]. We shall come back to this technical consideration in section 3.7 .

\subsection{Loop model formulation}

We shall refer to (6) as the cluster representation of $P_{B}(q, v)$. Below we shall use an equivalent formulation in terms of a loop model [28] defined on the medial lattice $\mathcal{M}(B)$. To this end, consider the two possible states of an edge $e=(i j) \in E$ between to adjacent vertices $i, j$ in $B$ :
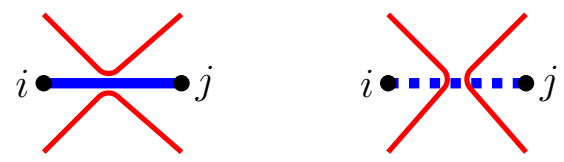

$\dagger$ Note the analogy to how distinct grey squares were glued at their terminals in order to form the basis $B$. 
In the left picture we have $e \in A$ and the edge is drawn as a thick blue line. The corresponding loops (thin red lines) reflect off the edge $e$. In the right picture we have $e \notin A$ and the edge is shown in dashed line style. The loops then cut through $e$, or equivalently, they reflect off the corresponding dual edge $e^{*}$. In both cases ( $e \in A$ and $e \notin A)$ the loops separate the clusters in $G_{A}=(V, A)$ from the dual clusters in $G_{A^{*}}=\left(V^{*}, A^{*}\right)$, where by definition $A^{*}$ consists of the edges dual to those in $E \backslash A$.

In a transfer matrix formalism the lattice is built up, row by row, starting at the bottom and moving towards the top. Each row is in turn built up, edge by edge, starting at the left and moving towards the right. One can think of this as sweeping some imaginary $d-1$ dimensional surface over the lattice in a number of discrete time steps. We refer to this surface as the time slice. Note that since we are in $d=2$ dimensions it is actually just a curve. More precisely, it is a horizontal line each time a row of the lattice has been completed, and a horizontal line with a kink when the row is only partially completed. At each step, the partially built lattice (the portion below the time slice) is characterised by the connectivity state of the clusters or loops intersecting the time slice, in a precise way that makes it possible to compute the partition function - or, in our case, the conditional partition functions entering in (6) - in the transfer process. The possible configurations of the edges ( $e \in A$ and $e \notin A$ ) must be summed over, and each term in the sum induces a definite operation on the connectivity states.

A detailed description of the transfer matrix formalism in the cluster representation was given in [14]. We here need the corresponding loop representation, describing the states and operations on the thin red lines in (7). We begin with a brief outline, deferring a more precise description of a number of important points to the following subsections.

For a planar graph one may use [28] the Euler relation to rewrite the partition function (1) in the loop representation as

$$
Z=q^{|V| / 2} \sum_{A \subseteq E} x^{|A|} n_{\text {loop }}^{\ell(A)},
$$

where $x=v / \sqrt{q}$, and $\ell(A)$ denotes the number of closed loops induced by the configuration $A$. The loop fugacity is $n_{\text {loop }}=\sqrt{q}$. Note that because of $(7)$ there is a local bijection between configurations of clusters and loops, so we may use the notation $A \subseteq E$ to specify the configurations of loops as well.

Consider then adding a single edge to the lattice. Imagining for the moment that transfer direction is upwards (the lattice is built from the bottom to the top), we shall refer to the horizontal edge in (7) as a "space-like" edge. When $e \notin A$ (right picture) the two loop strands just go though and nothing happens, while for $e \in A$ (left picture) the two strands are being connected and a new partially completed loop is started out. The sum over both possibilities can be described by an operator acting on adjacent loop strands at positions $i$ and $i+1$ :

$$
\mathrm{H}_{i}=\mathrm{I}+x \mathrm{E}_{i},
$$

where $\mathrm{E}_{i}$ are the generators of the Temperley-Lieb algebra [31] defined by

$$
\mathrm{E}_{i}^{2}=n_{\text {loop }} \mathrm{E}_{i},
$$




$$
\begin{aligned}
& \mathrm{E}_{i} \mathrm{E}_{i \pm 1} \mathrm{E}_{i}=\mathrm{E}_{i}, \\
& \mathrm{E}_{i} \mathrm{E}_{j}=\mathrm{E}_{j} \mathrm{E}_{i} \text { for }|i-j|>1 .
\end{aligned}
$$

The algebraic relations (10) can be proved graphically by gluing several diagrams of the type (7) on top of one another.

For a vertical, or "time-like" edge, we would have the graphical correspondence
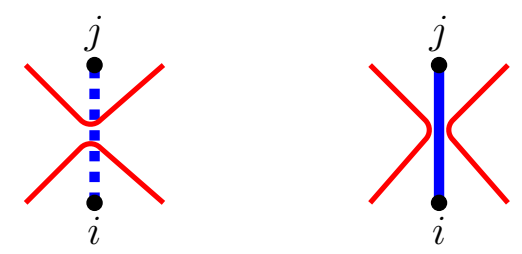

and since the situation $e \in A$ now corresponds to the right picture, the corresponding operator is

$$
\mathrm{V}_{i}=x \boldsymbol{I}+\mathrm{E}_{i} .
$$

In the following subsections we explain in detail how to adapt the loop representation to the geometry of Figure 2 and use it to compute the graph polynomial (6) rather than the full partition function $Z$. In particular, we explain how to discard configurations contribution to $Z_{1 \mathrm{D}}$, distinguish topologically the contributions to $Z_{2 \mathrm{D}}$ and $Z_{0 \mathrm{D}}$, and attribute to each diagram the correct powers of $x=v / \sqrt{q}$ and $n_{\text {loop }}=\sqrt{q}$. Based on this, the graph polynomial (6) can the be retrieved by changing the $\left(n_{\text {loop }}, x\right)$ variables back to $(q, v)$ and providing the overall factor $q^{|V| / 2}$ in (8).

\subsection{Four-terminal representation}

The graph polynomial $P_{B}(q, v)$ must be computed in the square-basis geometry (cf. Figure 2) which in the loop representation takes the appearance shown in Figure 3. The loops live on the medial lattice $\mathcal{M}(B)$, of which a part is shown as red and blue edges in Figure 3. In a nomenclature inspired by the theory of quantum integrable systems, we shall refer to the (red) horizontal edges as "auxiliary spaces" and the (blue) vertical edges as "quantum spaces".

The part of $\mathcal{M}(B)$ inside the grey squares depends of course on the lattice being studied. We denote it symbolically by the letter $R$. The operator constructing the corresponding part of the lattice is called the $\check{R}$-matrix and is written $\check{\mathrm{R}}_{i}$. It acts on two auxiliary spaces $(i, i+1)$ coming from the left and two quantum spaces $(i+2, i+3)$ coming from the bottom of the grey square, and produces outgoing quantum spaces $\left(i^{\prime}, i^{\prime}+1\right)$ on the top and auxiliary spaces $\left(i^{\prime}+2, i^{\prime}+3\right)$ on the right of the grey square. This labeling of spaces is shown in Figure 4.

For each of the lattices to be studied in this paper, $\check{\mathrm{R}}_{i}$ is a definite product of the elementary operators $\mathrm{H}_{j}, \mathbf{V}_{j}$ and $\mathrm{E}_{j}$ defined above, with $j=i, i+1, i+2$. This means in practice that the computation of $P_{B}(q, v)$ can be adopted to any desired lattice that can be shown to have the four-terminal structure of Figure 3 and for which the algebraic expression for $\check{\mathrm{R}}_{i}$ can be provided (see section 4 ). 


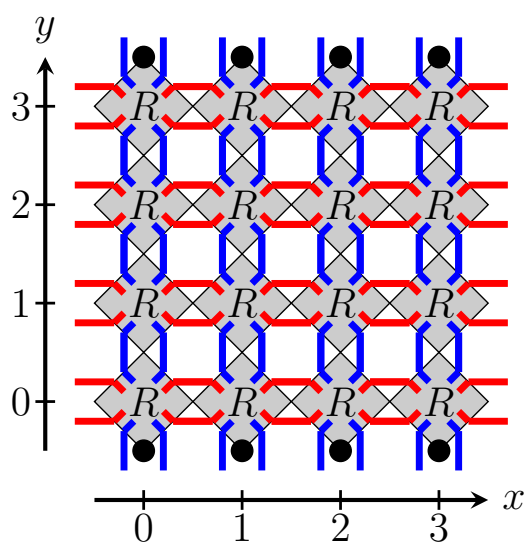

Figure 3. Square basis of size $n \times n$ with $n=4$ in the loop representation. Terminals of the basis are shown as black circles and periodic boundary conditions have been imposed horizontally. The loops live on the auxiliary and quantum spaces, shown in red and blue colour respectively. An $\check{R}$-matrix acts inside each grey square.

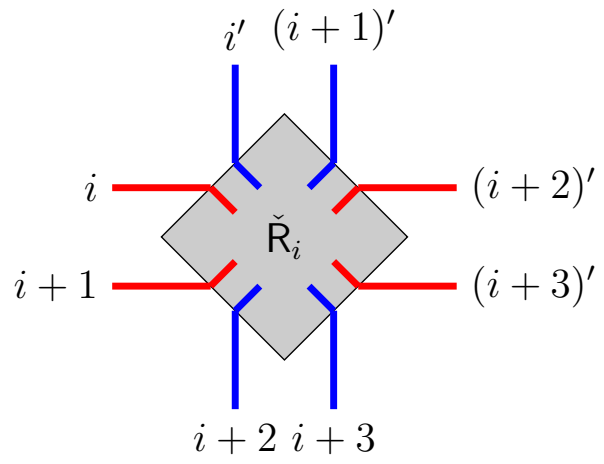

Figure 4. Labeling of the auxiliary and quantum spaces around an $\check{R}$-matrix.

To add one row of grey squares to the lattice, one inserts a pair of auxiliary spaces, acts with the product $\check{\mathrm{R}}_{2 n-2} \cdots \check{\mathrm{R}}_{4} \check{\mathrm{R}}_{2} \check{\mathrm{R}}_{0}$, and removes the pair of auxiliary spaces. The next subsection contains the precise definitions of the connectivity states, and describes how the elementary operators act on them, and how auxiliary spaces are inserted and removed.

Note that the labeling of Figure 4 implies that time flows in the North-East direction $\dagger$ and not simply upwards as we assumed for pedagogical reasons when discussing (7) and (11). This has an incidence on the interpretation of "horizontal" and "vertical", since these geometrical notions have now been rotated $45^{\circ}$ in the clockwise direction. To be precise, the epithet horizontal, or space-like (resp. vertical, or time-like) now means perpendicular (resp. parallel) to the direction of time flow. For instance, the $\check{R}$-matrix that constructs the square lattice is written

$$
\check{\mathrm{R}}_{i}=\mathrm{H}_{i+1} \mathrm{~V}_{i} \mathrm{\vee}_{i+2} \mathrm{H}_{i+1} \text {. }
$$

$\dagger$ This situation will be familiar to readers acquainted with the theory of quantum integrable systems. 


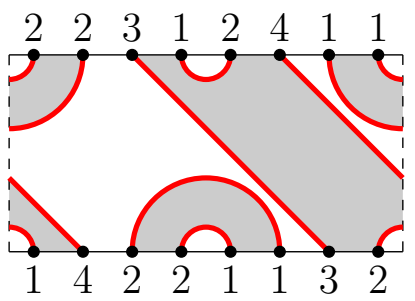

(a)

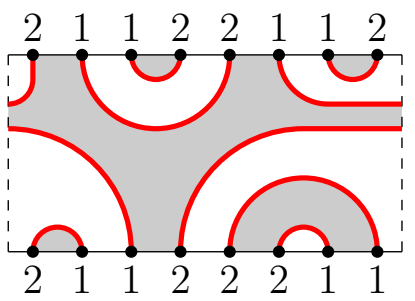

(b)

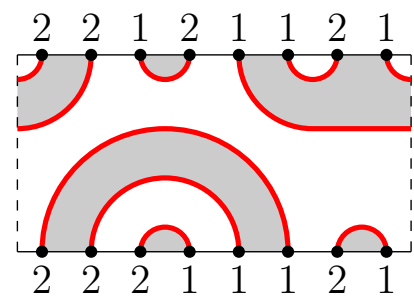

(c)

Figure 5. Three examples of connectivity states for $n=4$. The numbers along the two time slices provide a canonical coding of the connectivity state, as explained in the main text. Loops are shown as red solid lines. The corresponding clusters live in the areas shaded in grey, whilst the dual clusters live in the white areas.

Many more examples will be given in section 4 .

As already mentioned it is possible also to perform a restricted set of operations in the white squares. Since after completing a row of grey squares the auxiliary spaces are no longer at our disposal, this is essentially limited to letting the operator $\mathrm{H}_{i}$ act on the two quantum spaces within a white square in Figure 3. This has the effect of adding a horizontal diagonal to the white square.

\subsection{State space}

Since the terminals on the top and bottom of Figure 3 must eventually be glued together we actually need a set of two time slices. The first one is a horizontal line at the bottom of the system $\left(y=-\frac{1}{2}\right)$, and the second one is a horizontal line (with a kink when a row is only partially completed) that keeps moving upwards (and the kink moving to the right, i.e., in the "North-East direction", while completing a given row) until the lattice is completed. After completion the two time slices are glued together in a precise way (see section 3.7) that distinguishes the contributions to $Z_{2 \mathrm{D}}$ and $Z_{0 \mathrm{D}}$.

3.5.1. Description of the states It is convenient first to describe the state space for a complete row. In this case each of the time slices is a horizontal line that intersects the $2 n$ quantum spaces. No auxiliary spaces are involved in this case. A connectivity state describes how these $4 n$ intersection points are pairwise connected by the loop strands.

In Figure 5 we show a few examples of connectivity states. We call arc a loop segment that connects two points within the same time slice, and string a loop segment that connects points on different time slices. The number of strings is denoted $s$. Boundary conditions are periodic in the horizontal direction, as shown by the dashed sides of the construction boxes in Figure 5 .

Suppose that the points are labelled $0,1,2, \ldots, 2 n-1$ on the bottom time slice and $0^{\prime}, 1^{\prime}, 2^{\prime}, \ldots,(2 n-1)^{\prime}$ on the upper time slice. To relate the cluster and loop representations, it is important to observe that points with an even label have a cluster on the right and a dual cluster on the left (and vice versa for the points with an odd 
label). The clusters (resp. dual clusters) corresponding to the connectivity states in Figure 5 are shown in grey (resp. white) shading. This relation to clusters imposes important restrictions on the connectivities of loops. First, arcs connect points of opposite parities. Second, strings connect points of the same parity. In particular one may define a string to be even or odd, depending on the parity of the points that it connects. Third, $s$ is even and there are as many even as odd strings. When $s=0$, consider the configuration of arcs restricted to just one of the two time slices. We call this configuration closed if the equivalent clusters are bounded away from the other time slice (in which case one dual cluster will connect the two time slices), and open if one cluster connects the two time slices (in which case the dual clusters are bound). Then, fourth, when $s=0$ a connectivity state consists of two open arc configurations, or of two closed arc configurations.

These concepts are illustrated in Figure 5. Figure 5a shows a state with $s=2$. The case of $s=0$ is depicted in Figure 5b for two open arc states, and in Figure 5c for two closed arc states. The figures also exemplify a coding of the states which turns out to be convenient for describing the transfer matrix algorithm. On the time slice on the top of the system, the leftmost (resp. rightmost) point of an arc carries the code 1 (resp. 2). Arcs on the bottom of the system are coded similarly, but after a $180^{\circ}$ rotation. Strings are coded as pairs of matching codes $(3,4, \ldots)$.

3.5.2. Action of Temperley-Lieb generators The connectivity states provide a representation of the periodic Temperley-Lieb algebra $T L_{2 n}\left(n_{\text {loop }}\right)$, which is faithful for generic values of $n_{\text {loop }}$ (see e.g. Ref. [32]). However, to obtain a finite-dimensional algebra we need to take a quotient (closely related to the so-called Jones-Temperley-Lieb algebra [33]) by

(i) identifying states in which the strings connect the same points on the top and bottom time slices, but wind a different number of times around the periodic (horizontal) direction, and

(ii) giving a definite weight $n_{\text {wind }}$ to each loop that winds the periodic direction.

To take into account the first condition, we choose (for $s>0$ ) to draw the string whose anchoring point on the top time slice is the furthest to the left so that it does not cross the periodic direction. The remaining strings are then drawn in the unique (up to isotopy) way that respects planarity (i.e., arcs and strings do not cross). This convention at the same time provides a canonical coding of the strings: the anchoring points on the top time slice are labelled $3,4,5, \ldots, s+2$ from left to right, and the labels on the bottom time slice follow by matching the codes of the points that are connected by a string. The canonical coding then provides a unique description of the states in the quotient algebra.

Notice that any diagram with a winding loop will contribute to $Z_{1 \mathrm{D}}$. We must therefore set $n_{\text {wind }}=0$, and this at the same time provides a valid choice for the 
second condition. However, setting just $n_{\text {wind }}=0$ is not sufficient for getting rid of all contributions to $Z_{1 \mathrm{D}}$, so more work will be required (see section 3.7).

The Temperley-Lieb generator $\mathrm{E}_{i}$, shown graphically in the left picture in (7) and (11), now acts on the connectivity states by contracting the strands at neighbouring positions $i$ and $j=i+1 \bmod 2 n$ on the top time slice, and subsequently liking those two points by a new arc (meaning that the points $i$ and $j$ acquire the codes 1 and 2 respectively). The precise meaning of the contraction depends on the codes $\left(c_{i}, c_{j}\right)$ of the points prior to the action by $\mathrm{E}_{i}$ :

(i) If $\left(c_{i}, c_{j}\right)=(1,2)$ a loop is formed, and the weight $n_{\text {loop }}$ must be applied.

(ii) If $\left(c_{i}, c_{j}\right)=(1,1)$ the partner of $c_{j}$ has its code changed from 2 to 1 .

(iii) If $\left(c_{i}, c_{j}\right)=(2,2)$ the partner of $c_{i}$ has its code changed from 1 to 2 .

(iv) If $\left(c_{i}, c_{j}\right)=(2,1)$ the codes are unchanged, but if $c_{i}$ is the partner of $c_{j}$ a winding loop is formed, and the weight $n_{\text {wind }}=0$ must be applied.

(v) If $c_{i}>2$ is a string and $c_{j} \leq 2$ is an arc, then the partner of $c_{j}$ becomes the new position of the string, and hence has its code changed to $c_{i}$. The same statement holds true with $i$ and $j$ interchanged.

(vi) If both of $\left(c_{i}, c_{j}\right)$ are strings, the partner of $c_{i}$ has its code changed to 2 and the partner of $c_{j}$ gets the code $1 . \dagger$

Note that in all of these cases, except the last one, the number of strings is conserved by $\mathrm{E}_{i}$, so that the codes on the bottom time slice do not change at all. But in the last case a pair of strings is destroyed and their anchoring points on the bottom time slice are turned into an arc.

3.5.3. Dimension of the transfer matrix To determine the dimension of the transfer matrix we must count the number of states. We still suppose for the time being that the uppermost row is complete (i.e., there are no auxiliary spaces).

By cutting all the strings (if any) a connectivity state describing the full system of two time slices is transformed into a pair of reduced states each associated with one of the time slices. The reduced states consist of arcs and half strings. When $s=0$ the reduced states can be characterised as open or closed, just like the full states.

It is easy to count the number of reduced states. For $s=0$, out of the $2 n$ points there are $n$ with code 1 and $n$ with code 2 . A moment's reflection reveals that all the $\left(\begin{array}{c}2 n \\ n\end{array}\right)$ possible placements of these codes correspond to a valid state. For $s=2 k>0$ one can similarly convince oneself that specifying the $n-k$ points with code 1 will uniquely imply the positions of the arcs and half strings. So there are $\left(\begin{array}{c}2 n \\ n-k\end{array}\right)$ reduced states in general.

Conversely, a pair of reduced states with the same number of half strings can be transformed into a full connectivity state by gluing pairs of half strings. However, for

$\dagger$ Note that this respects the convention that arcs on the bottom time slice have the code 2 (resp. 1) assigned to their leftmost (resp. rightmost) point, viz., the convention obtained by rotating through $180^{\circ}$ rotation the one used for arcs on the top time slice. 
$s=0$ one obtains a valid state only by "gluing" (or rather juxtaposing, since nothing is actually being glued!) two open or two closed half states. Since the set of open and closed half states are bijectively related by performing a cyclic shift, there are $\frac{1}{2}\left(\begin{array}{c}2 n \\ n\end{array}\right)$ of each. Moreover, for $s=2 k>0$ strings the gluing can be done in $k$ inequivalent ways, since each half string must be glued to one of the same parity and the cyclic order of strings must be respected. These observations imply that there are

$$
\operatorname{dim}(n)=\frac{1}{2}\left(\begin{array}{c}
2 n \\
n
\end{array}\right)^{2}+\sum_{k=1}^{n} k\left(\begin{array}{c}
2 n \\
n-k
\end{array}\right)^{2}
$$

connectivity states.

3.5.4. Bijection between states and integers To write an efficient transfer matrix algorithm it is desirable to possess a bijection between the integers $0,1,2, \ldots, \operatorname{dim}(n)-1$ and the states coded as in Figure 5. This is straightforwardly done provided that one can provide a canonical ordering of the states.

The states can be ordered according to the following criteria:

(i) The number of strings $s=2 k$ with $k=0,1, \ldots, n$

(ii) The reduced connectivity state on the bottom time slice.

(iii) The cyclic rotation involved in gluing $2 k$ half strings on the top time slice to $2 k$ half strings on the bottom time slice. Note that with the canonical coding of Figure 5 this amounts to shifting cyclically the codes $>2$ on the bottom time slice.

(iv) The reduced connectivity state on the top time slice.

Since the reduced connectivity states have a simple interpretation in terms of binomial coefficients, they can easily be endowed with a canonical ordering (e.g. by ordering them lexicographically). Alternatively, since the number of reduced states is much less than the total number of states, we can simply generate the reduced states by hashing techniques and endow them with some ad hoc ordering, such as their position in the hash table.

The practical implementation of the bijection in terms of the above criteria, and the ordering of the reduced states, is most conveniently written in terms of various tables, as outlined in $[34,35]$ for a couple of related situations.

3.5.5. Handling auxiliary spaces To handle a partially completed row of the lattice we need to be able to insert and remove auxiliary spaces. We also need to count the number of states in the presence of $p$ auxiliary spaces. (The four-terminal representation requires $p=0,1,2$ but extensions of the formalism to higher values of $p$ may turn out to be of interest for lattices which cannot be cast in the four-terminal form.)

Suppose now that the $n$ points in the top time slice are initially labelled from left to right:

$$
\begin{array}{llllll}
\mathbf{I} & \mathbf{I} & \mathbf{I} & \mathbf{I} & \mathbf{I} \\
0 & 1 & 2 & 3 & \cdots & 2 n-1
\end{array}
$$


The bottom time slice simply occupies the $2 n$ labels following those of the top time slice, and plays no further role in the following construction. Accordingly we shall not represent it here.

Inserting the first (lower) auxiliary space amounts to adding two extra points to the left of those in the top time slice. This can be considered as two copies of the same point which are initially connected. We have now:

$$
\begin{aligned}
& 2 n+1=0
\end{aligned}
$$

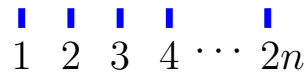

The $2 n$ points of the quantum spaces have had their labels shifted by one, in order to accommodate the labels 0 and $2 n+1$ of the quantum spaces. In order to connect the latter two points, and respect the conventions for an arc on the top time slice, we attribute to them the codes $c_{2 n+1}=1$ and $c_{0}=2$.

Similarly, the second (upper) auxiliary space is inserted by adding a pair of points with codes 1 and 2 in-between those previously inserted. This looks like:

$$
\begin{aligned}
& 2 n+3=0 \\
& 2 n+2=1
\end{aligned}
$$

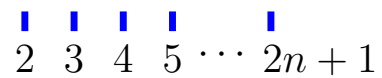

Note that the quantum space labels have again been shifted by one, as have those of the first (lower) auxiliary space. This is necessary for respecting the cyclic order of the labels upon moving around the top time slice. So the complete set of four points inserted to the left of those in the top time slice have the codes $c_{2 n+2}=c_{2 n+3}=1$ and $c_{0}=c_{1}=2$. To add one complete row of grey squares to the lattice, one then applies the product of operators $\check{\mathrm{R}}_{2 n-2} \cdots \check{\mathrm{R}}_{4} \check{\mathrm{R}}_{2} \check{\mathrm{R}}_{0}$. Note that before the action with the factor $\check{\mathrm{R}}_{i}$ the two rightmost dangling ends of the auxiliary spaces carry the labels $i$ and $i+1$, in agreement with the conventions of Figure 4.

After the application of the last factor, $\check{\mathrm{R}}_{2 n-2}$, the situation is as follows:

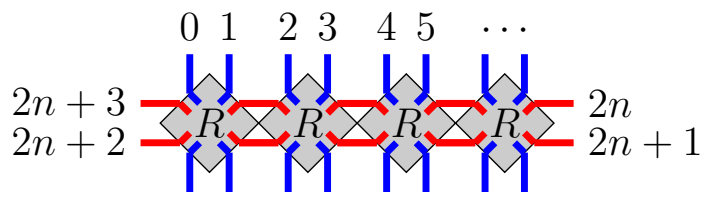

The row is basically completed, and the new quantum spaces have come out with the correct labeling $0,1,2, \ldots, 2 n-1$. However, the two auxiliary spaces remain open, so the dangling ends on the left and the right will have to be glued and then removed. To this end, one applies the "contraction" operator (discussed above when defining the Temperley-Lieb generators $\mathrm{E}_{i}$ ) to identify first points $2 n+1$ and $2 n+2$, and then $2 n$ and $2 n+3$. The four auxiliary points are then removed from the state, and we are ready to start all over and add a new row to the lattice. 


\begin{tabular}{l|rrr|r}
$n$ & $\operatorname{dim}(n, 0)$ & $\operatorname{dim}(n, 1)$ & $\operatorname{dim}(n, 2)$ & Ref. $[14]$ \\
\hline 1 & 3 & 10 & 35 & 14 \\
2 & 36 & 132 & 490 & 1430 \\
3 & 500 & 1900 & 7245 & 208012 \\
4 & 7350 & 28420 & 109956 & 35357670 \\
5 & 111132 & 433944 & 1693692 & 6564120420 \\
6 & 1707552 & 6708240 & 26332020 & 1289904147324 \\
7 & 26501904 & 104535288 & 411945105 & 263747951750360 \\
\hline
\end{tabular}

Table 2. Dimension $\operatorname{dim}(n)=\operatorname{dim}(n, 0)$ of the transfer matrix for a completed row, and $\operatorname{dimensions} \operatorname{dim}(n, p)$ for a partially completed row with $p=1,2$ auxiliary spaces, for the sizes $n$ used in section 4 . This is compared with the dimension of the transfer matrix of Ref. [14].

The number of states $\operatorname{dim}(n, p)$ in the presence of $p$ auxiliary spaces is an obvious generalisation of (14). We find that

$$
\operatorname{dim}(n, p)=\frac{1}{2}\left(\begin{array}{c}
2 n+2 p \\
n+p
\end{array}\right)\left(\begin{array}{c}
2 n \\
n
\end{array}\right)+\sum_{k=1}^{n} k\left(\begin{array}{c}
2 n+2 p \\
n+p-k
\end{array}\right)\left(\begin{array}{c}
2 n \\
n-k
\end{array}\right)
$$

We have tabulated these dimensions in Table 2 for the values of $n$ and $p$ used in the computations of section 4 . These dimensions should be compared with those of the transfer matrix approach of Ref. [14]. In order to compute $P_{B}(q, v)$ for an $n \times n$ square basis, [14] used states which are planar partitions of the $N=4 n$ terminal points in Figure 2. The number of such states is given by the Catalan number

$$
\operatorname{Cat}(N)=\frac{1}{N+1}\left(\begin{array}{c}
2 N \\
N
\end{array}\right)
$$

The advantage of the present approach, as announced in the Introduction, is to reduce this to $\operatorname{dim}(n, 2)$, thus raising the limit of practical feasibility of the computations from $n_{\max }=4[14]$ to $n_{\max }=7$. This improvement is achieved by the dealing efficiently with the horizontal periodic boundary conditions, leading to the reduction from $4 n$ to $2 n$ of the number of terminals implied by Figure 3.

The bijection between states and integers extends straightforwardly to the case with auxiliary spaces, the only difference being that the reduced state describing the top time slice contains $2 p$ extra points.

\subsection{Implementational details}

We now describe some considerations on how to efficiently build the lattice by repeated applications of the transfer matrix. The general prescription for building a single row has been detailed in section 3.5.5:

(i) Insert two auxiliary spaces;

(ii) Add a row of grey squares, each corresponding to the application of an operator $\check{\mathrm{R}}_{i}$; 
(iii) Remove the two auxiliary spaces;

(iv) (For certain lattices:) Add horizontal edges in the white square by application of the $\mathrm{H}_{i}$ operators.

At the beginning of the transfer process, the top and bottom time slices coincide. Therefore the initial state consists of just a single connectivity state $s_{0}$ in which, for each $i=0,1, \ldots, 2 n-1$, the points $i$ and $i+2 n$ are connected by a string. In the conventions of section 3.5.1 this means that the corresponding coding is $c_{i}=c_{i+2 n}=3+i$. In other words, the initial state is therefore a unit vector where $s_{0}$ has Boltzmann weight 1 , while all other states have weight 0 .

For the computation of $P_{B}(q, v)$, the states are represented as arrays of polynomials in the variables $\left(n_{\text {loop }}, x\right)$, with coefficients that are non-negative integers. The degrees of the polynomials are $|V|$ in the $n_{\text {loop }}$ variable and $|E|$ in the $x$ variable, where $|V|$ and $|E|$ denote the number of vertices and edges in $B$. For the computation of the percolation critical polynomial $P_{B}(1, v)$ it suffices obviously to employ arrays of polynomials in the single $x$ variable. And, finally, when we only desire to find the roots of $P_{B}(q, v)$ numerically, the arrays consist of real numbers to the desired numerical precision. In order to obtain the roots to (at least) 50 digits we use 100-digit real numbers and the Newton-Raphson method, where derivatives are computed from a first-order finitedifference formula with $\epsilon=10^{-50}$. In practice we use the CLN library [36] that efficiently handles such high-precision real numbers within our $\mathrm{C}++$ implementation of the algorithm.

In all cases, the arrays have dimensions $\operatorname{dim}(n, p)$ given by (15), where $p=0,1,2$ depending on the number of open auxiliary spaces.

The bijection described in section 3.5.4 is employed throughout the transfer process to translate back and forth between connectivity states (on which the action of the fundamental Temperley-Lieb operators $\mathrm{E}_{i}$ has been detailed in section 3.5.2) and integers $0,1, \ldots, \operatorname{dim}(n, p)-1$ that specify the position in the arrays of the relevant Boltzmann weights.

In the cases where the polynomials $P_{B}(q, v)$ or $P_{B}(1, v)$ are computed exactly, the coefficients of the polynomials are very large integers for all but the smallest values of the size $n$. Although the CLN library [36] offers also arbitrary-precision integers, it is more efficient to compute the result modulo a sufficient number of different primes $p_{i}$ and reconstitute the exact result from the Chinese remainder theorem. The standard version of our algorithm (i.e., the one used throughout section 4) employs only additions, whereas the "generic Ř-matrix" version described in section 6.1 uses also multiplications. Since standard unsigned integers in $\mathrm{C}++$ lie in the range $0,1, \ldots, 2^{32}-1$ we therefore take $p_{i}<2^{31}$ in the former case and $p_{i}<2^{16}$ in the latter. The most demanding computations required of the order of 20 different primes within this scheme.

Some consideration on the application of an $\check{\mathrm{R}}_{i}$ operator are also in order. For most lattices - including all those of section 4-we can express $\check{\mathrm{R}}_{i}$ as a product of the elementary operators $\mathrm{H}_{i}, \mathrm{~V}_{i}$ and $\mathrm{E}_{i}$. An example has been given in (13). In those 
cases it is usually numerically most efficient to apply each of the factors separately, i.e., to build the lattice one edge at a time. (This procedure is known as sparse-matrix factorisation.) Note that the application of each of these elementary operators to a given input connectivity state produces only two output states. Expanding out the product $\check{\mathrm{R}}_{i}$ by hand would lead instead to up to fourteen output states (since the number of planar pairings of the eight points appearing in Figure 4 is $\operatorname{Cat}(4)=14$ ), and with vastly more complicated coefficients. However, in some cases not all 14 output states are actually generated. This is so in particular for the kagome lattice, where only 13 states are generated. Accordingly some of the states stored in the arrays will have zero weight. For instance, for the kagome lattice we find that only $37 \%$ of the $\operatorname{dim}(n, 2)$ states carry non-zero weight for $n=1$, but this occupation ratio increases to $74 \%$ for $n=2,87 \%$ for $n=3$ and $90 \%$ for $n=4$. It is therefore hardly worth dealing with this slight waste of memory resources in this case.

However, in other situations the occupation ratio may go instead to zero for large $n$. This is notably the case for some of the site percolation problems of section 7 . It is then more efficient to avoid storing a lot of zero coefficients in the array, but rather insert the states that are really generated (with non-zero weight) in a hash table. In particular, the bijection of section 3.5.4 becomes superfluous. We shall describe the hashing version of the algorithm in more details in sections 6.1 and 7 .

\subsection{Topological considerations}

When the basis $B$ has been completely built up, it remains to discuss how to actually compute the graph polynomial, for which we recall the definition (6):

$$
P_{B}(q, v)=Z_{2 \mathrm{D}}-q Z_{0 \mathrm{D}}
$$

The end result of the transfer process is a linear combination of connectivity states involving two time slices, such as those shown in Figure 5, with coefficients that are polynomials in $n_{\text {loop }}$ and $x$. Each state is described by its number in the canonical ordering, from which the coding (i.e., the integers $c_{i}$ shown in Figure 5) can be inferred from the bijection of section 3.5.4. Also, from this coding, one can rather straightforwardly construct a representation of the pairing of the $4 n$ points (namely $2 n$ on each time slice) induced by the loops (shown in red in Figure 5) which allows, in particular, to "travel" along the loops.

The goal is now to identify, for each connectivity state, the top and bottom time slices such that the $i$ th point on the top time slice gets glued to the $i$ th point on the bottom time slice. Within this gluing procedure we should, on one hand, be able to distinguish which states contribute to $Z_{2 \mathrm{D}}, Z_{1 \mathrm{D}}$ and $Z_{0 \mathrm{D}}$ (those of $Z_{1 \mathrm{D}}$ are then discarded, since they do not contribute to $\left.P_{B}(q, v)\right)$ and, on the other hand, provide some extra powers of $n_{\text {loop }}=\sqrt{q}$ that have not been accounted for by the transfer process.

To count the number of loops $P$ and, at the same time, determine whether there is a loop of non-trivial homotopy, we begin by travelling along each loop. To this end, one 
starts at some initial reference point and follows its arc or string to the partner point. Then one jumps to the opposite time slice (since the two are glued) and repeats the process until one comes back to the initial point. During this travel, a list of the points visited is maintained, as well as the horizontal and vertical winding numbers, $w_{x}$ and $w_{y}$, incurred. Note that $w_{x}$ changes as the result of an arc or string explicitly crossing the periodic boundary condition, whereas $w_{y}$ changes when one jumps from one time slice to the other. If $\left(w_{x}, w_{y}\right) \neq(0,0)$ the loop has non-trivial homotopy, and we are dealing with a state that contributes to $Z_{1 \mathrm{D}}$ and therefore can be discarded. Note that all non-trivial loops (if any) necessarily have the same homotopy, i.e., the same values of $\left(w_{x}, w_{y}\right)$ up to a global sign change.

Having completed the travel along the first loop, if a non-visited point still exists, this is taken as the new reference point, and we trace out the next loop. This process terminates when all points have been visited. We now know the number of loops $P$.

For example, the states in Figures $5 \mathrm{~b}$ and $5 \mathrm{c}$ both have a loop with $\left(w_{x}, w_{y}\right)=(1,0)$. This is easiest seen by considering the loop in Figure 5b (resp. Figure 5c) that passes through the third (resp. second) point from the left on the bottom time slice.

Suppose now that we have found that all $P$ loops in the state have trivial homotopy. This is the case for the state in Figure 5a, which we shall henceforth use as an example. We then need to find out if the state contributes to $Z_{2 \mathrm{D}}$ or to $Z_{0 \mathrm{D}}$. To this end we use a variant of the Euler relation, i.e., we compute the quantity

$$
\chi=E+2 C-V-P,
$$

where the quantities $E, C$ and $V$ will be defined below.

Corresponding to a loop configuration (red lines in Figure 5) there is a corresponding cluster configuration (grey shading in Figure 5). The cluster configuration can be seen as a hyper graph on the set $\mathcal{P}_{1}$ of $2 n$ points (namely $n$ on each time slice) situated to the right of the (loop) point $i$ and to the left of point $i+1$, for all even $i$. An area with grey shading containing $d+1$ points of the hyper graph is called a hyper edge of degree $d$. (Note that in the definition of hyper edges we do not impose the identification of the top and bottom time slices.) Let now $E$ be the sum of the degrees $d$ of all hyper edges. One may think of $E$ as the equivalent number of usual (not hyper) edges. For instance, the state in Figure 5 a can be represented as:

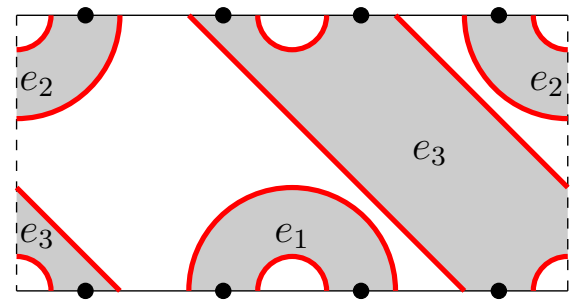

It has three hyper edges: $e_{1}$ and $e_{2}$ each of degree 1 , and $e_{3}$ of degree 3 . Therefore $E=1+1+3=5$.

Let us provide a few details on how to construct the cluster configuration from the loop configuration. The connections between (cluster) points within a single time slice 
(top or bottom) can be easily inferred by travelling along the arcs on that time slice. It is more delicate to infer the connections (if any) from one time slice to the other. It is obvious that if there are $s=2 k>0$ strings, there will be $k$ such connections, and these can again be easily inferred by travelling along the strings (this is the case in Figure 5a). However, if $s=0$ the existence of connections between the clusters on the top and bottom time slices depends on whether the two reduced states are both closed or both open (see section 3.5.1). An example involving open states is provided by the following figure:

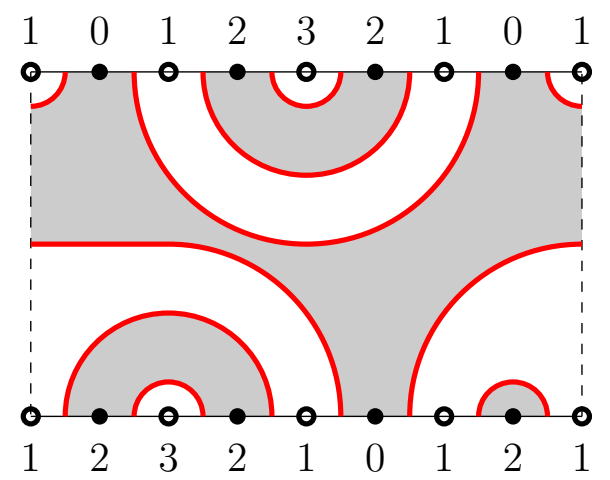

To determine in general the connections (if any) between the clusters on the top and bottom time slices we proceed as follows. Consider first the set $\mathcal{P}=\mathcal{P}_{1} \cup \mathcal{P}_{2}$ consisting of $2 n$ points on each time slice, which is the union of the points $\mathcal{P}_{1}$ on which the clusters live -i.e., those with an even loop point on the left and an odd loop point on the right, shown as solid circles in (19) - and the points $\mathcal{P}_{2}$ on which dual clusters live - i.e., those with an odd loop point on the left and an even loop point on the right, shown as open circles in (19). We now define a set of integer "heights" on $\mathcal{P}$ as follows. Starting from an arbitrary initial value (chosen as 1 in (19)), and moving along the top (resp. bottom) time slice from left to right (resp. from right to left), let the height increase (resp. decrease) by one unit each time one crosses a loop opening (resp. closing), i.e., a loop point with code $c_{i}=1$ (resp. $c_{i}=2$ ). Since only height differences are defined, this procedure defines the heights only up to a global translation. The heights corresponding to the example (19) are shown next to each point in $\mathcal{P}$. It is easy to see that if, for each of the time slices taken separately, the minimum of this height profile (which is 0 in (19) for both time slices) resides at a point of $\mathcal{P}_{1}$ (resp. $\mathcal{P}_{2}$ ), the corresponding reduced state is open (resp. closed). In the case of a pair of open reduced states, all the points of $\mathcal{P}_{1}$ residing at the minimum height on the top time slice are incident on the same hyper edge as the corresponding points of minimum height on the bottom time slice. In the example (19) there are two such points on the top time slice and one on the bottom time slice, so top and bottom are connected through a hyper edge of degree 2. This concludes the construction of the cluster configuration from the loop configuration.

We finally define $V$ and $C$ as, respectively, the number of vertices and clusters in the hyper graph. Both of these number are defined after the identification of the top and bottom time slices. In particular $V=n$. Either of the states (18) and (19) turn out 
to have $C=1$. We can then compute $\chi$ from (17), and we find $\chi=5+2-4-3=0$ for (18) and $\chi=4+2-4-1=1$ for (19).

In general the possible values are $\chi=0,1,2$. When $\chi=0$ the state belongs to the $Z_{0 \mathrm{D}}$ class, and when $\chi=1$ or 2 it belongs to the $Z_{2 \mathrm{D}}$ class. Moreover, when $\chi=1$ (resp. $\chi=2$ ) we can deduce that the state contains $s=2 k>0$ strings (resp. $s=0$ strings), but we shall not need this fact to compute $(6)$.

Now that the nature $\left(Z_{0 D}, Z_{1 \mathrm{D}}\right.$ or $\left.Z_{2 \mathrm{D}}\right)$ of each connectivity state has been determined, it remains only to multiply it by a certain power of $n_{\text {loop }}$ that has not been accounted for by the transfer process itself. First, there is a factor of $\sqrt{q}=n_{\text {loop }}$ for each vertex in the basis $B$, coming from the front factor of (8). The number of vertices should of course be computed up to the identification which is made by imposing the doubly periodic boundary conditions on $B$ (i.e., gluing the left and right, and the top and bottom). Second, each state has to be multiplied by $n_{\text {loop }}^{P}$, where we recall that $P$ is the number of loops in the final state. Finally, the $Z_{2 \mathrm{D}}$ configurations should be multiplied by a factor of $q=n_{\text {loop }}^{2}$; this follows from the Euler relation.

\section{Results on Archimedean lattices}

In this section we present our results for the Potts model (and the special case of bond percolation) on the Archimedean lattices. The hexagonal lattice can be omitted from the discussion since it is the dual of the triangular lattice, $\left(6^{3}\right)=D\left(3^{6}\right)$, and hence covered by the general remarks on duality made in section 5 .

The triangular and square lattices are of the three-terminal type and hence exactly solvable. This means that the critical curves are exactly known [37, 5, 38]. The exact solvability will cause $P_{B}(q, v)$ to shed a small factor $[13,14]$, corresponding to the exact critical curves. However, the remaining, large factor in $P_{B}(q, v)$ will still give important information about additional critical behaviour in the antiferromagnetic region $v<0$. This information - and the whole critical manifold of the other eight Archimedean lattices - is only rendered approximately by the roots of the critical polynomials, but the accuracy is such that we can use powerful extrapolation techniques to obtain the ferromagnetic critical points to very high precision, along with a precise global understanding of the phase diagram in the antiferromagnetic region.

An important feature in the regime $v<0$ is the presence of a so-called BerkerKadanoff $(\mathrm{BK})$ phase [39]. This is a region in the real $(q, v)$ plane throughout which correlation functions decay as power laws, and where the temperature variable $v$ is irrelevant in the renormalisation group $(\mathrm{RG})$ sense. The lower and upper boundaries of the BK phase are a pair of antiferromagnetic transition curves, $v_{-}(q)<v<v_{+}(q)$, that merge at some value $q_{\mathrm{c}}$ :

$$
\lim _{q \rightarrow q_{\mathrm{c}}} v_{-}(q)=\lim _{q \rightarrow q_{\mathrm{c}}} v_{+}(q) .
$$

The inequality $q_{\mathrm{c}} \leq 4$ is guaranteed by quantum group results [39]. For several latticesincluding the square lattice - one has $q_{\mathrm{c}}=4$ exactly $[39,14]$, but there are indications 


\begin{tabular}{l|lll} 
Lattice & Vertices & Edges & Parity of $n$ \\
\hline Triangular & 2 & 6 & Any \\
Square & 2 & 4 & Any \\
Kagome & 3 & 6 & Any \\
Four-eight & 4 & 6 & Any \\
Frieze & 2 & 5 & Even \\
Three-twelve & 6 & 9 & Any \\
Cross & 3 & $\frac{9}{2}$ & Even \\
Snub square & 2 & 5 & Even \\
Snub hexagonal & $\frac{12}{7}$ & $\frac{30}{7}$ & 0 mod 7 \\
& 3 & $\frac{15}{2}$ & Even \\
Ruby & 3 & 6 & Even \\
\hline
\end{tabular}

Table 3. Number of vertices and edges per grey square (cf. Figure 2) for each Archimedean lattice, using square bases of size $n \times n$ grey squares. In addition we state any parity constraint on $n$. Note that we have two different ways of constructing the snub hexagonal lattice.

that on other lattices - including the kagome lattice - one may have $q_{\mathrm{c}}<4$ strictly [14]. The RG irrelevance of $v$ has the consequence that phase transitions inside the BK phase are $v$-independent and will manifest themselves as vertical rays in the $P_{B}(q, v)=0$ manifold. It was found in $[13,14]$ for several examples that these vertical rays occur when $q$ is equal to a Beraha number

$$
B_{k}=(2 \cos (\pi / k))^{2}
$$

with even $k=4,6,8, \ldots$, but when $q_{\mathrm{c}}<4$ the range of $k$-values is limited by $B_{k}<q_{\mathrm{c}}$.

The results given below provide firm evidence that these characteristics of the BK phase are generic for the Potts model defined on any two-dimensional lattice. Moreover, we obtain precise information about the extent of the BK phase and the value of $q_{\mathrm{c}}$ for all the lattices under study.

On a more technical level, we show below how each of the Archimedean lattices can be cast as a four-terminal lattice, in the precise sense of Figure 2. This is done notably by specifying the corresponding $\check{R}$-matrix. In cases where this construction is not unique, the best choice for our purposes is the one that allows $n$ to take any value (i.e., with no parity constraints) and that packs as many vertices and edges as possible into the basis of a given size $n$. To quantify this latter aspect, we show in Table 3 the number of vertices and edges per $\check{R}$-matrix that were achieved for each lattice (we include any horizontal diagonals on the white squares in this count). For instance, our largest $(n=7)$ computation on the three-twelve lattice uses a basis of $6 n^{2}=294$ vertices and $9 n^{2}=441$ edges.

For $n \leq 5$ (resp. $n=6$ ) we have computed the exact critical polynomial for the Potts model (resp. for bond percolation only). Our plots of the phase diagrams are based on these polynomials. As in our preceding work $[21,14]$ the polynomials are 


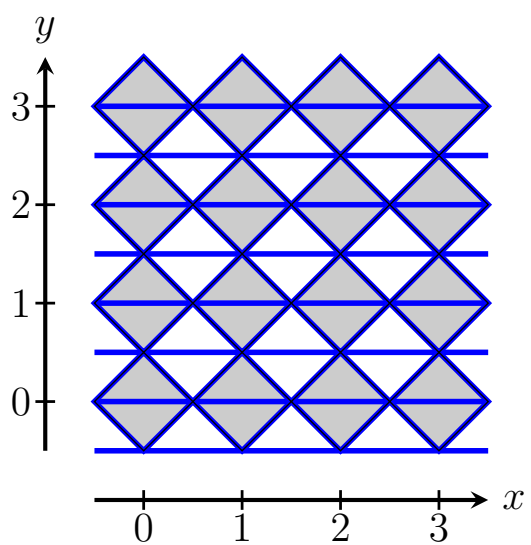

Figure 6. Four-terminal representation of the triangular lattice.

available in electronic form as supplementary material to this paper. $\dagger$ The degree of $P_{B}(q, v)$ is $k_{q} n^{2}$ in the $q$-variable and $k_{v} n^{2}$ in the $v$-variable, where $k_{q}$ and $k_{v}$ can be read from the second and third columns of Table 3. For instance, our largest $(n=5)$ two-variable polynomial for the three-twelve lattice has degree 150 in $q$, and degree 225 in $v$. Moreover, the coefficients are typically 60 -digit integers.

\subsection{Triangular lattice $\left(3^{6}\right)$}

A square basis for the triangular lattice is obtained by placing the following $\check{R}$-matrix

$$
\check{\mathrm{R}}_{i}=\mathrm{H}_{i+1} \mathrm{~V}_{i} \mathrm{H}_{i+1} \mathrm{~V}_{i+2} \mathrm{H}_{i+1}
$$

inside each grey square in Figure 2. In addition we need horizontal diagonals on all the white squares. The resulting representation is shown in Figure 6. There are 4 vertices and 6 edges per grey square.

The critical polynomials $P_{B}(q, v)$ invariably factorises for any size $n$ of the basis, shedding the small factor

$$
P_{\text {tri }}(q, v)=v^{3}+3 v^{2}-q .
$$

This is compatible with the fact [37] that the triangular-lattice Potts model is exactly solvable on the curve $P_{\text {tri }}(q, v)=0$. In particular, for $q=1$ we have the root $v=-1+2 \cos (2 \pi / 9)$, meaning that the exact percolation threshold is

$$
p_{\mathrm{c}}=\frac{v}{1+v}=2 \sin \left(\frac{\pi}{18}\right) \text {. }
$$

The remaining, large factor in $P_{B}(q, v)$ gives additional information about the critical manifold in the regime $v<0$, as we shall now see. Its roots in the real $(q, v)$ plane are shown in Figure 7. The lower boundary of the BK phase, denoted $v_{-}(q)$, is the lower branch of the cubic (23). The corresponding upper boundary $v_{+}(q)$ can be seen

$\dagger$ This text file PB.m provided can be processed by MATHEMATICA or - maybe after minor changes of formatting - by any symbolic computer algebra program of the reader's liking. 


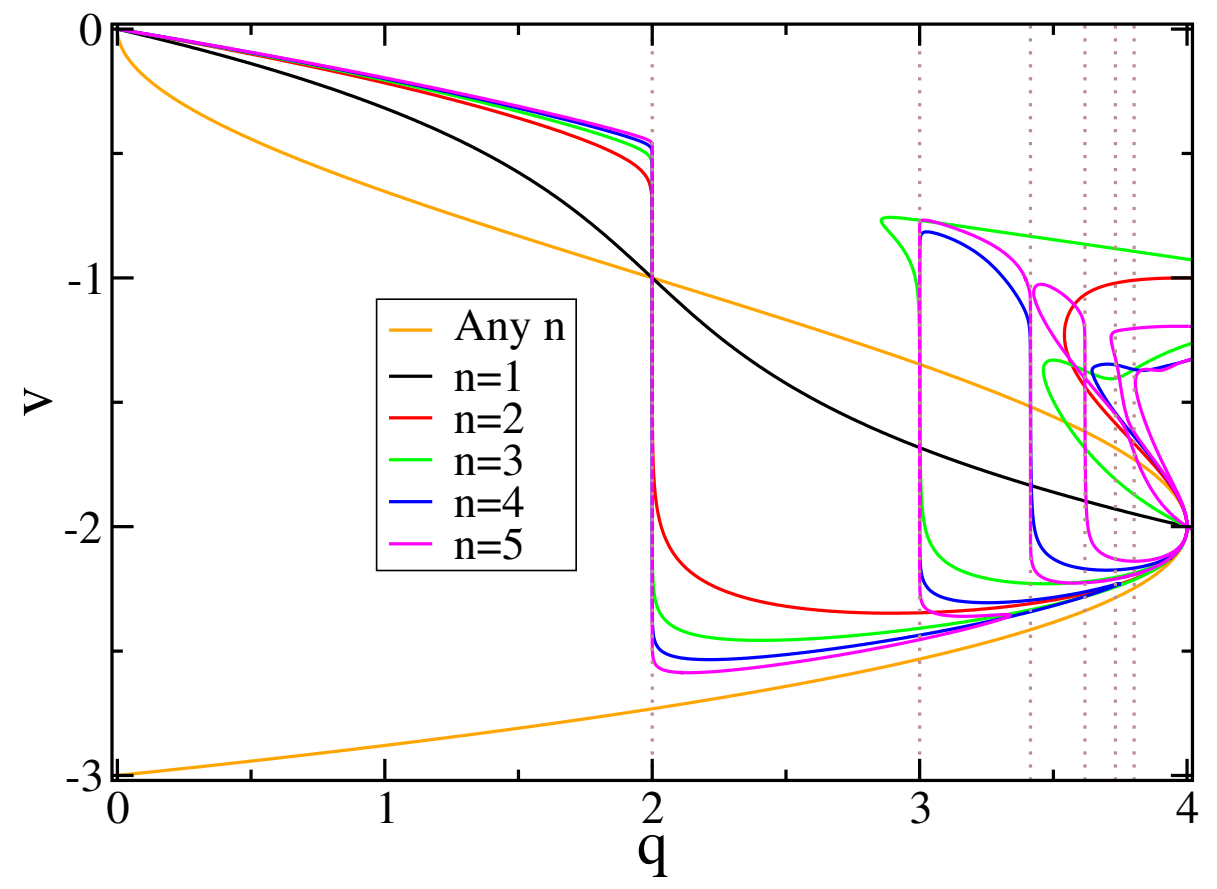

Figure 7. Roots of $P_{B}(q, v)$ for the Potts model on the triangular lattice, using $n \times n$ square bases. The curve labelled "any $n$ " corresponds to (23).

in Figure 7 as the curve starting from the origin with near-horizontal slope, passing through the top point of a series of vertical rays, and extending towards the special point $(q, v)=(4,-2)$. Curiously, the critical polynomials miss the part of $v_{+}(q)$ with $2<q<3$. Heuristically, this is "because" the polynomials have to trace out both $v_{+}(q)$, $v_{-}(q)$ and the vertical rays in a zig-zag fashion that becomes increasingly complicated upon approaching $q=4$. The vertical rays corresponding to $k=4,6,8,10$ in (21) - and to a lesser extent $k=12,14$ as well-are clearly visible from the figure. Here and in the following, we help the visual identification of such vertical rays by superimposing a number of dotted grey lines on the figures.

In conclusion, Figure 7 provides rather compelling evidence that the curves $v_{ \pm}(q)$ will merge in $(q, v)=(4,-2)$. In particular $q_{\mathrm{c}}=4$ for the triangular lattice. It follows almost inevitably that the critical curve to which the BK phase is RG-attracted must be the middle branch of (23). That conclusion is backed up by a number of other studies $[40,41,42,43]$.

We should mention that the triangular-lattice Potts model is exactly solvable on the chromatic line $v=-1[44,45,46]$. It follows from $[45,46]$ that $v_{+}(q)=-1$ for some value $q=3.819671731 \cdots$ obtained by equating two infinite products. The region near $(q, v)=(4,-1)$ exhibits some rather complicated physics and would be a suitable subject for a separate study [47]. 


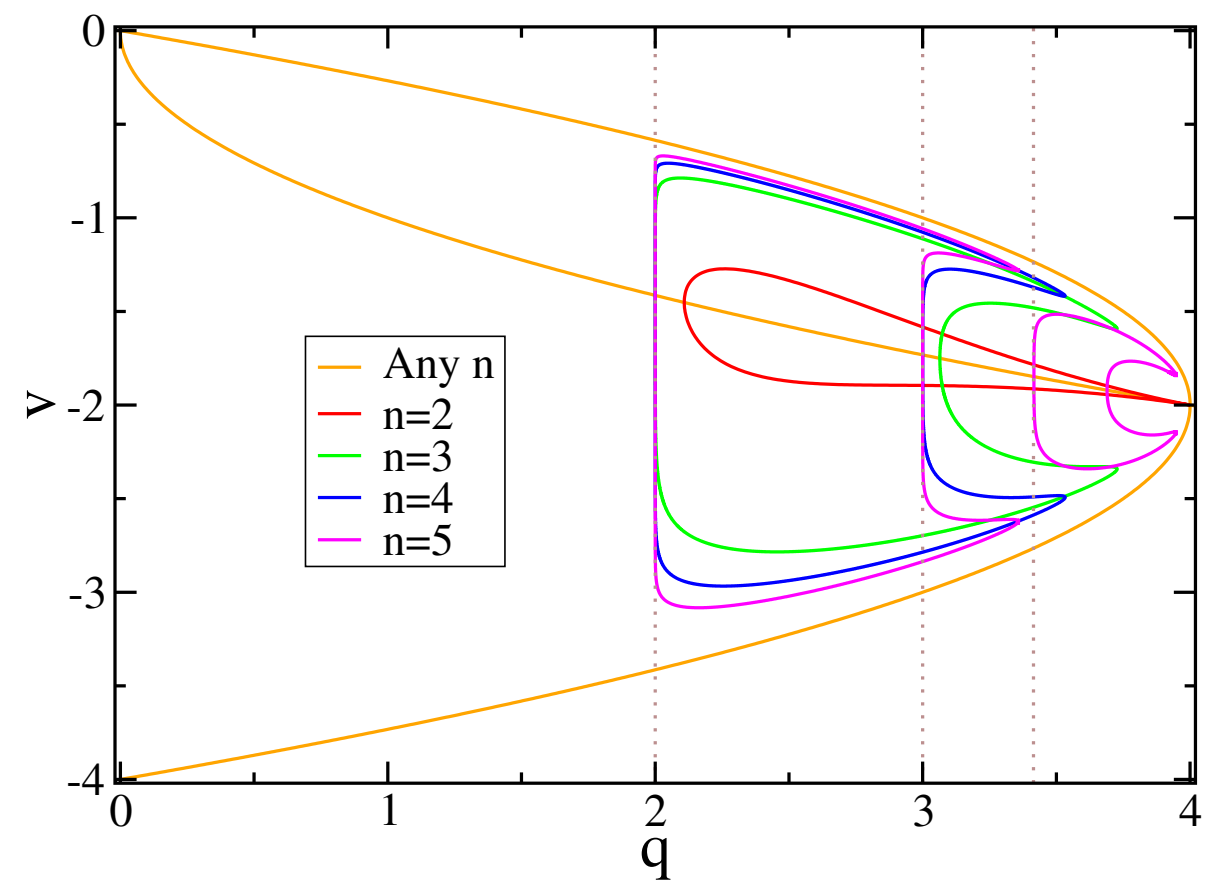

Figure 8. Roots of $P_{B}(q, v)$ for the Potts model on the square lattice, using $n \times n$ square bases. The curves labelled "any $n$ " correspond to (26).

\subsection{Square lattice $\left(4^{4}\right)$}

The square lattice can obviously be obtained by removing the diagonal edges from the triangular lattice. The $\check{R}$-matrix then reads

$$
\check{\mathrm{R}}_{i}=\mathrm{H}_{i+1} \mathrm{~V}_{i} \mathrm{~V}_{i+2} \mathrm{H}_{i+1} \text {. }
$$

The basis then looks like Figure 2 with the horizontal edges removed. There are 2 vertices and 4 edges per grey square.

For any size $n$, the critical polynomial $P_{B}(q, v)$ factorises, shedding two small factors:

$$
P_{\mathrm{sq}}(q, v)=\left(v^{2}-q\right)\left(v^{2}+4 v+q\right) .
$$

The zero set of the first factor describes the selfdual critical point of the square-lattice Potts model [5], while the zero set of the second factor yields two mutually dual antiferromagnetic critical points [38]. The model is exactly solvable on these curves $[5,38,43,48,49]$. In particular, we find that $P_{\mathrm{sq}}(1,1)=0$, so the exact percolation threshold is

$$
p_{\mathrm{c}}=\frac{1}{2} \text {. }
$$

The roots of $P_{B}(q, v)$ in the real $(q, v)$ plane are shown in Figure 8; the curves with $n \leq 4$ were already reported in [21]. Of all the Archimedean lattices, the square lattice is the only one where this phase diagram can be claimed to be completely understood. The boundaries of the BK phase are given by the second factor in (26), namely

$$
v_{ \pm}(q)=-2 \pm \sqrt{4-q},
$$




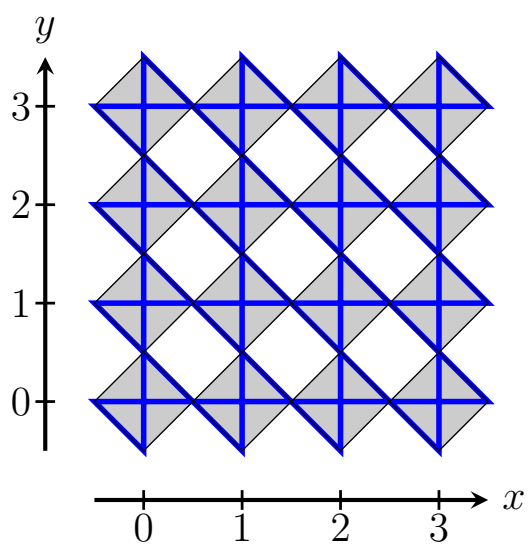

Figure 9. Four-terminal representation of the kagome lattice.

and in particular $q_{\mathrm{c}}=4$. In the thermodynamical limit, $n \rightarrow \infty$, we expect an infinite set of vertical rays, corresponding to (21) with $k=4,6,8, \ldots$. The first few, with $k=4,6,8$, are clearly visible in our results for $n \leq 5$, shown in Figure 8 , as is the precursor of the $k=10$ ray (which is not yet in its correct position).

\subsection{Kagome lattice $(3,6,3,6)$}

The square and triangular lattices (and the hexagonal lattice, which is the dual of the triangular) could have been presented in three-terminal form. This fact actually makes it possible to compute the critical manifolds, $P_{\mathrm{sq}}(q, v)=0$ and $P_{\text {tri }}(q, v)=0$, exactly [11], and it is closely related to the exact solvability [5, 38, 43, 48, 37] of the models along these curves.

However, the kagome lattice - and indeed all the remaining Archimedean latticesis not of the three-terminal type. Accordingly no exact solution is known to this day. The graph polynomial method therefore gives only approximate results, which are however very accurate, in particular in the ferromagnetic region $v>0$.

The $\check{R}$-matrix of the kagome lattice can be written as

$$
\check{\mathrm{R}}_{i}=\mathrm{H}_{i+1} \mathrm{~V}_{i+2} \mathrm{~V}_{i} \mathrm{E}_{i+1} \mathrm{~V}_{i+2} \mathrm{~V}_{i} \mathrm{H}_{i+1} \text {. }
$$

The resulting representation is shown in Figure 9. There are 3 vertices and 6 edges per grey square.

We have computed the (unique) root $P_{B}(q, v)$ in the ferromagnetic regime, $v>0$, for several integer values of $q$. The results for $q=1$ are shown in Table 4 to 50-digit numerical precision, in terms of the percolation probability $p=\frac{v}{1+v}$, for square bases of size $1 \leq n \leq 7$. A quick glance at the table makes it obvious that these numbers converge very fast to their expected limit $p_{\mathrm{c}}$.

The roots for $1 \leq n \leq 4$ have already been reported in [21], leading the authors to propose a final estimate of $p_{\mathrm{c}}=0.52440500(1)$. However, the fact that we now have three more terms in the sequence allows us to employ powerful extrapolation techniques 


\begin{tabular}{l|l}
$n$ & $p_{\mathrm{c}}$ \\
\hline 1 & 0.52442971752127479354687968153445507162056741657866 \\
2 & 0.52440672318823181914323447999258988541033371409674 \\
3 & 0.52440517271376997270613021015286282832593143635602 \\
4 & 0.52440502742741472069907568050523723911941122246320 \\
5 & 0.52440500598061634783869324699127606806572366674050 \\
6 & 0.52440500130658104881349494422717375226544897430878 \\
7 & 0.52440499997320890049536436452361870395440274892056 \\
\hline$\infty$ & $0.524404999173(3)$ \\
Ref. $[25]$ & $0.52440499(2)$
\end{tabular}

Table 4. Bond percolation threshold $p_{\mathrm{c}}$ on the kagome lattice.

to obtain a very accurate final value of $p_{\mathrm{c}}$. We have chosen to apply the time-proven Bulirsch-Stoer (BS) extrapolation [50]. This algorithm requires a parameter $w$ which can be thought of as a correction-to-scaling exponent. In an preliminary step we obtain an approximate value for $w$ from a non-linear fit of the data in Table 4 to the form

$$
p_{\mathrm{c}}(n)=p_{\mathrm{c}}+A n^{-w} .
$$

The best results are obtained by constraining this fit to the last three available data points. We do not report the values of $w$ found for each data set to be considered in this paper, except for the first few examples of each type of problems. We shall however provide a few general remarks in the Discussion section 8. Generally speaking we find $w \approx 6$ for the best behaved (bond or site) percolation problems. Obviously, the higher the value of $w$, the better will be the precision on the final result.

In a second step, we insert the value found for $w$ into the implementation of the BS algorithm described by Monroe [51]. This results in a series of approximants that are compared among themselves in order to assess a final value and error bar. We crosscheck our results by repeating the whole procedure with the last data point being eliminated, in order to ensure that the central value and error bar obtained from fits on $N-1$ points are compatible (albeit of course less precise) with those obtained on all $N$ data points.

For some of the lattice for which fewer data points (i.e., sizes $n$ ) are available, some adaptations of this general procedure will be necessary. We shall return to this in the following subsections.

In Table 4 and the following many tables in this paper, we compare our final result with the most accurate value known from previous numerical work. In the present case, we find $w \approx 6.36$, and the relative precision on the final value of $p_{\mathrm{c}}$ is of the order $4 \cdot 10^{-11}$, that is, four orders of magnitude better than the previous result [25].

We next turn to the Ising model $(q=2)$. In this case, all the $P_{B}(q, v)$ are found to factorise into small factors. The maximum degree of the factors is $d_{\max }=4$ for $n=1,2$; $d_{\max }=8$ for $n=3,4$; and $d_{\max }=16$ for $n=5$. There is precisely one of these factors, 


\begin{tabular}{l|l}
$n$ & $v_{\mathrm{c}}$ \\
\hline 1 & 1.8762692083457608448172661268682642135309588452285 \\
2 & 1.8764397543028806860142570871053207225112352007846 \\
3 & 1.8764569161964147459134636690080036024897511662923 \\
4 & 1.8764589940034619711814113716932244874728362773156 \\
5 & 1.8764593952716922296679157122640055912513566938545 \\
6 & 1.8764595053053275343456174924063602310856281037794 \\
7 & 1.8764595432649855348163610191948592418994287446958 \\
\hline$\infty$ & $1.8764595734(3)$ \\
Ref. [24] & $1.87646(5)$
\end{tabular}

Table 5. Critical point $v_{\mathrm{c}}$ of the $q=3$ state Potts model on the kagome lattice.

\begin{tabular}{l|l}
$n$ & $v_{\mathrm{c}}$ \\
\hline 1 & 2.1558422365136376068815817932185116250325673239278 \\
2 & 2.1562074529907952231032843370607657724546027337305 \\
3 & 2.1562475983381240731591377965181755879281862607822 \\
4 & 2.1562528801542168626963668658051048225566594172221 \\
5 & 2.1562540028309456631273970233783408172592478843687 \\
6 & 2.1562543392791356268847391898132756614686640239252 \\
7 & 2.1562544649475050404839402645792684169318045633213 \\
\hline$\infty$ & $2.1562545798(8)$ \\
Ref. [24] & $2.1561(5)$
\end{tabular}

Table 6. Critical point $v_{\mathrm{c}}$ of the $q=4$ state Potts model on the kagome lattice.

namely

$$
-8-8 v+4 v^{3}+v^{4}
$$

that possesses a positive root,

$$
v_{\mathrm{c}}=\sqrt{3+2 \sqrt{3}}-1 \simeq 1.542459756 \cdots .
$$

Moreover, (31) is a factor in $P_{B}(q, v)$ for any size $n$. Its physical root (32) coincides with the exactly known critical point of the kagome-lattice Ising model [52, 23]. Below we shall similarly see that we recover exact results for the Ising model on any lattice.

The polynomials for the Ising model are often found to simplify under the change of variables $v=-1+\sqrt{y}$. Recall that $v=\mathrm{e}^{K}-1$, but rewriting the nearest neighbour interaction energy as in Ising form, $K \delta_{\sigma_{i}, \sigma_{j}}=K_{\text {Ising }}\left(S_{i} S_{j}+1\right)$ for spins $S_{i}= \pm 1$, we find $K=2 K_{\text {Ising }}$ so that $y=\mathrm{e}^{K_{\text {Ising }}}$ is simply the Boltzmann factor for a pair of aligned Ising spins. In the present case (31) simplifies to

$$
-3-6 t+t^{2} \text {. }
$$




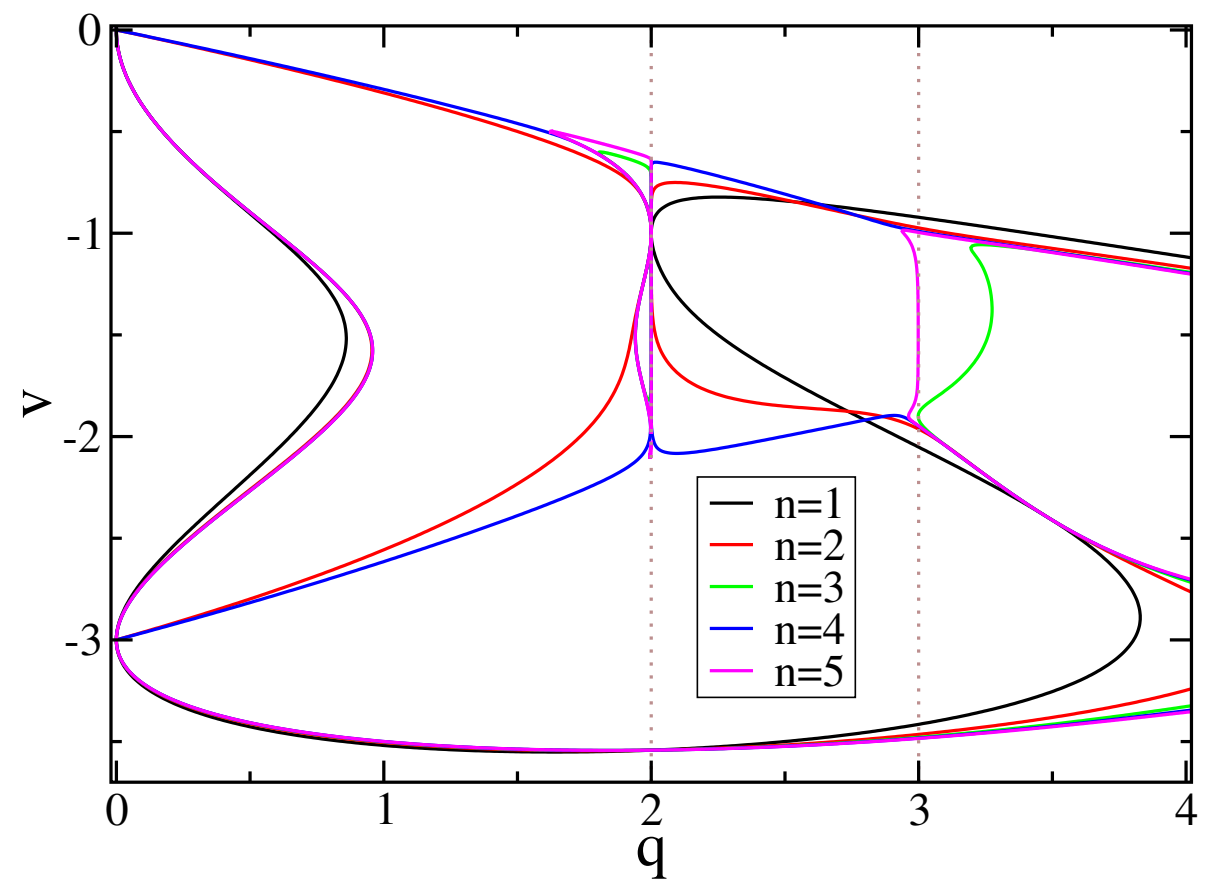

Figure 10. Roots of $P_{B}(q, v)$ for the Potts model on the kagome lattice, using $n \times n$ square bases.

The critical points for the $q=3$ and $q=4$ state Potts models are shown in Tables $5-6$. The exponent appearing in $(30)$ is $w \approx 5.36$ for $q=3$, and $w \approx 4.80$ for $q=4$. In any case, using BS extrapolations as explained above, we arrive at values for $v_{\mathrm{c}}$ which are considerably more accurate than previous numerical results. Note in particular that numerical simulations of the Monte Carlo or transfer matrix type are usually particularly difficult for $q=4$ because of the presence of logarithmic corrections to scaling. By contrast, the graph polynomial method only experiences a slight decrease of $w$, and the precision is almost as good as for percolation. Thus, for $q=4$ our final value for $v_{\mathrm{c}}$ is six orders of magnitude more precise than the previous result [24].

The phase diagram on the kagome lattice has been discussed in detail in [13], and further in [14] based on square-basis $P_{B}(q, v)$ with $n=1,2,3,4$. In Figure 10 we show again the roots of $P_{B}(q, v)$ in the real $(q, v)$ plane, but this time with the $n=5$ basis included. Because of the extensive treatment of this phase diagram in $[13,14]$ we shall be rather brief.

The BK phase contains vertical rays at $q=B_{4}=2$ and $q=B_{6}=3$. Unlike the triangular and square lattices, there is no sign of the BK phase widening out towards $q=4$ as $n$ increases. Its rightmost termination might be close to the $n=3$ arc extending to around $q \approx 3.2$. This arc is confirmed by results from the hexagonal bases studied in [14]. On the other hand, even allowing for $n$ mod 2 parity effects which are visible elsewhere in the phase diagram, it is curious that this arc is not confirmed by the $n=5$ critical polynomial. So it might also be that the BK phase in fact terminates right at the $q=3$ vertical ray. In any case, it seems certain that $q_{\mathrm{c}}<4$ for the kagome lattice. 


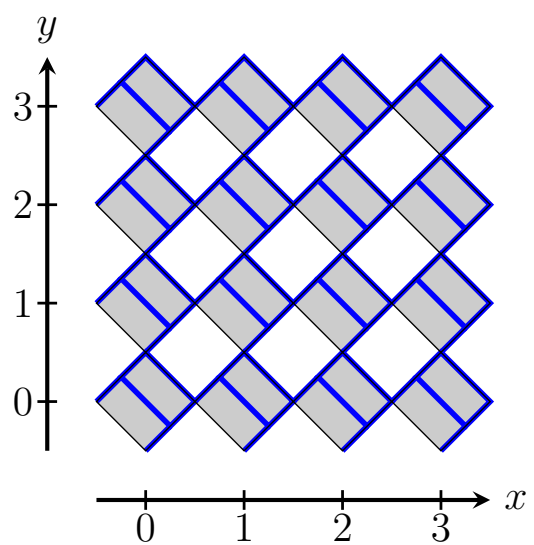

Figure 11. Four-terminal representation of the four-eight lattice.

The upper boundary $v_{+}(q)$ of the BK phase is the near-straight line emanating from the origin and passing through the point $(q, v)=(3,-1)$. Indeed, the three-state zerotemperature antiferromagnet on the kagome lattice is equivalent to the corresponding four-state model on the triangular lattice, which in turn is known to be critical with central charge $c=2$ [53]. It is interesting to observe that at finite $n$ the curve $v_{+}(q)$ is approximated by various pieces from the different $P_{B}(q, v)$, and that no single critical polynomial reproduces the curve completely. This is in line with observations already made in $[13,14]$. In particular we see clear $n$ mod 2 parity effects: the critical polynomials with even $n$ (resp. odd $n$ ) are the only ones to produce the part of $v_{+}(q)$ with $0<q \lesssim 1.6$ and $2<q<3$ (resp. $1.6 \lesssim q<2$ ).

Meanwhile, the lower boundary $v_{-}(q)$ of the BK phase is the near-straight line emanating from $(q, v)=(0,-3)$ and passing through $\approx(3,-2)$. It is determined by the polynomials with even $n$. Interestingly there is a lower-lying curve, coming out from $(0,-3)$ with infinite slope, and the space between this curve and $v_{-}(q)$ is devoid of vertical rays (because it does not belong to the BK phase).

Finally, the existence of two small enclosed regions, or phases - the first a thin sliver between $(2,-2)$ and $(2,-1)$, and the other a triangular-shaped region above $(2,-1)$-is confirmed by the new $n=5$ polynomial.

\subsection{Four-eight lattice $\left(4,8^{2}\right)$}

A four-terminal representation of the four-eight lattice is shown in Figure 11. The corresponding $\check{R}$-matrix reads

$$
\check{\mathrm{R}}_{i}=\mathrm{V}_{i+2} \mathrm{~V}_{i} \mathrm{H}_{i+1} \mathrm{~V}_{i+2} \mathrm{~V}_{i} \mathrm{H}_{i+1} .
$$

There are 4 vertices and 6 edges per grey square.

The approximations to the bond percolation threshold $p_{\mathrm{c}}$ are given in Table 7 . Since also for this lattice any parity of $n$ is possible, the BS extrapolation scheme produces very accurate values, improving considerably on the existing numerical results which 


\begin{tabular}{l|l}
$n$ & $p_{\mathrm{c}}$ \\
\hline 1 & 0.6768351988164058635685961953282386597701580217547 \\
2 & 0.6768110511337950640725367041515342178711568870560 \\
3 & 0.6768050108863651886629332178738395634982856516173 \\
4 & 0.6768036936560548693645165737411119820502567917852 \\
5 & 0.6768033435707186400895193695054260593374332806116 \\
6 & 0.6768032260648857547522627416623972342414983881124 \\
7 & 0.6768031780886579080959851245202783704998102947149 \\
\hline$\infty$ & $0.6768031269(6)$ \\
Ref. [54] & $0.6768023(6)$
\end{tabular}

Table 7. Bond percolation threshold $p_{\mathrm{c}}$ on the four-eight lattice.

\begin{tabular}{l|l}
$n$ & $v_{\mathrm{c}}$ \\
\hline 1 & 3.742119707930614518717609546330093725738120066089 \\
2 & 3.742406812389425084936236849313041587665529581274 \\
3 & 3.742474558548594455190569943534293743089719291957 \\
4 & 3.742488803421386923793990079403662434668070157084 \\
5 & 3.742492503198695522319538480078407840640750408712 \\
6 & 3.742493724307267658361207275220913244289197872944 \\
7 & 3.742494216612624056940208879007645446884063978949 \\
\hline$\infty$ & $3.742494730(5)$
\end{tabular}

Table 8. Critical point $v_{\mathrm{c}}$ of the $q=3$ state Potts model on the four-eight lattice.

are shown for comparison. We note that the exponent appearing in (30) comes out as $w \approx 4.28$ in this case, so it is definitely lattice dependent.

In the Ising case $(q=2)$ the polynomials $P_{B}(q, v)$ systematically factorise. The maximum degree of the factors is $d_{\max }=4$ for $n=1,2 ; d_{\max }=8$ for $n=3 ; d_{\max }=6$ for $n=4$; and $d_{\max }=16$ for $n=5$. There is precisely one of these factors, namely

$$
-4-8 v-6 v^{2}+v^{4}
$$

that possesses a positive root,

$$
v_{\mathrm{c}}=\frac{1+\sqrt{5+4 \sqrt{2}}}{\sqrt{2}} \simeq 3.015445388 \cdots,
$$

and this factor occurs in $P_{B}(q, v)$ for any size $n$. Its positive root (36) produces the exactly known critical point of the Ising model on the four-eight lattice [55, 23].

The $q=3$ and $q=4$ critical points $v_{\mathrm{c}}$ are shown in Tables $8-9$, and just like in the percolation case the BS extrapolation produces very accurate final values.

Ref. [14] already contained a discussion of the phase diagram for the four-eight lattice. But to highlight the new $n=5$ results, Figure 12 shows again the roots of $P_{B}(q, v)$ in the real $(q, v)$ plane. 
High-precision percolation thresholds and Potts-model critical manifolds

\begin{tabular}{l|l}
$n$ & $v_{\mathrm{c}}$ \\
\hline 1 & 4.367630831288118711619621980404618323651758096250 \\
2 & 4.368211338019043993502048035939247980189947635592 \\
3 & 4.368344356164380256004179009338136774148698456053 \\
4 & 4.368371674728465301875011853593201988010643386877 \\
5 & 4.368378652366770115169979832681074376730845677084 \\
6 & 4.368380925842698493907585059226287321316844017984 \\
7 & 4.368381832892126568493945658547177946678474675220 \\
\hline$\infty$ & $4.36838276(2)$
\end{tabular}

Table 9. Critical point $v_{\mathrm{c}}$ of the $q=4$ state Potts model on the four-eight lattice.

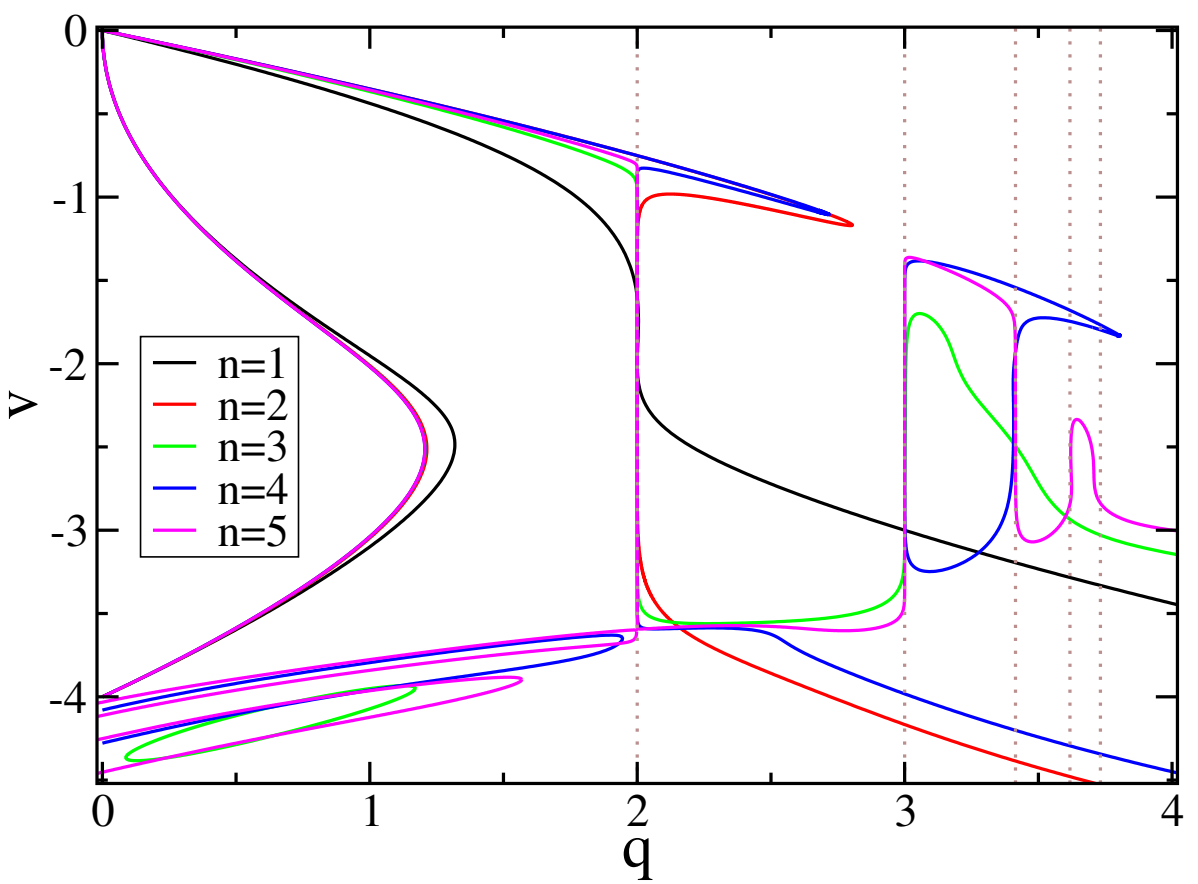

Figure 12. Roots of $P_{B}(q, v)$ for the Potts model on the four-eight lattice, using $n \times n$ square bases.

The boundaries of the BK phase can be clearly seen. The upper boundary $v_{+}(q)$ is the almost-straight line emanating from the origin and extending out towards $\approx(4,-2)$. The lower boundary starts at $\approx(0,-4)$. The zero sets of the various critical polynomials fill in the curves $v_{ \pm}(q)$ in a zig-zag fashion, while at the same time providing vertical rays at the Beraha numbers (21). The rays with $k=4,6,8,10,12$ are clearly visible in the figure. It is interesting to notice the formation of narrow "fingers" that tend to close those parts of the BK boundaries that are not provided by the principal "zig-zag" trend. For example, the lower boundary with $0<q<2$ is produced by fingers in the critical polynomials with $n \geq 3$. 


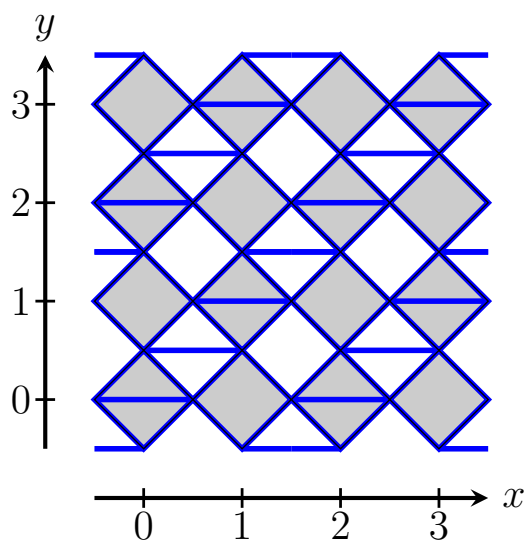

Figure 13. Four-terminal representation of the frieze lattice.

Overall, it seems likely that the BK phase for this lattice will extend all the way out to $q=4$, and so we can conjecture that $q_{\mathrm{c}}=4$ for the four-eight lattice.

\subsection{Frieze lattice $\left(3^{3}, 4^{2}\right)$}

The frieze lattice is the first example of a lattice which cannot be represented in fourterminal form by using the same $\tilde{\mathrm{R}}$-matrix in all grey squares $(x, y)$. Instead we have:

$$
\check{\mathrm{R}}_{i}= \begin{cases}\mathrm{H}_{i+1} \mathrm{~V}_{i} \mathrm{H}_{i+1} \mathrm{~V}_{i+2} \mathrm{H}_{i+1} & \text { for } x+y \text { even } \\ \mathrm{H}_{i+1} \mathrm{~V}_{i+2} \mathrm{~V}_{i} \mathrm{H}_{i+1} & \text { for } x+y \text { odd }\end{cases}
$$

In addition, there are horizontal diagonals on the white squares with coordinates

$\left(x+\frac{1}{2}, y+\frac{1}{2}\right)$ for $x+y$ even. The resulting basis, shown in Figure 13, needs $n$ to be even in order for it to produce the frieze lattice upon tiling. It has 2 vertices and 5 edges per grey square.

The restriction that $n$ need to be even is somewhat problematic for our approach. We have now only three data points $(n=2,4,6)$ for the extrapolations, instead of the usual seven $(n=1,2, \ldots, 7)$ when no parity constraint is operative. Fitting first the three data points to the form $(30)$ we find that $w \approx 3.03$. This three-point fit also provides the central value shown in Table 10. Next, based on other lattices for which more data points are available, we can estimate that this value of $w$ is likely to shift by around 1/10 upon increasing the size. Performing next a two-point fit to the two largest sizes, with a fixed value of $w$ within the range $3.03 \pm 0.10$, we get some alternative extrapolated values from which we can judge the size of the error bar on the central value.

Obviously this procedure leads to final results which are less precise than those of the preceding sections; but they still have a precision superior to that of existing numerical results.

The approximations for the percolation threshold $p_{\mathrm{c}}$ are shown in Table 10, and those for the critical points $v_{\mathrm{c}}$ for the $q=3$ and $q=4$ Potts models are given in 


\begin{tabular}{l|l}
$n$ & $p_{\mathrm{c}}$ \\
\hline 2 & 0.41963138921485282522020802393581141495177970672968 \\
4 & 0.41963933385520484749314071759551986794774805977647 \\
6 & 0.41964011556577399022999609393742526619676365808443 \\
\hline$\infty$ & $0.41964044(1)$ \\
Ref. [54] & $0.4196419(4)$
\end{tabular}

Table 10. Bond percolation threshold $p_{\mathrm{c}}$ on the frieze lattice.

\begin{tabular}{l|l}
$n$ & $v_{\mathrm{c}}$ \\
\hline 2 & 1.2060925735117353155747857952640056241243199926123 \\
4 & 1.2060633270192625886214470713076706696973022455589 \\
6 & 1.2060607188311827112162217288312021603769835821224 \\
\hline$\infty$ & $1.20605973(5)$
\end{tabular}

Table 11. Critical point $v_{\mathrm{c}}$ of the $q=3$ state Potts model on the frieze lattice.

\begin{tabular}{l|l}
$n$ & $v_{\mathrm{c}}$ \\
\hline 2 & 1.3761421373604738385559000230091418072845484528956 \\
4 & 1.3760828367736083803069388239875587480765505652209 \\
6 & 1.3760777196319081822843041399511989912220832991296 \\
\hline$\infty$ & $1.37607584(7)$
\end{tabular}

Table 12. Critical point $v_{\mathrm{c}}$ of the $q=4$ state Potts model on the frieze lattice.

Tables 11-12.

For the Ising model $(q=2)$ we find the now-familiar factorisation of the polynomials $P_{B}(q, v)$. The maximum degree of the factors is $d_{\max }=2$ for $n=2$ and $d_{\max }=12$ for $\mathrm{n}=4$. But the recurrent factor that determines the ferromagnetic critical point is simply

$$
-1+v
$$

so that

$$
v_{\mathrm{c}}=1 \text {. }
$$

in agreement with [23].

The obtainable information on the phase diagram also suffers from the parity restriction, since the polynomials $P_{B}(q, v)$ are now at our disposal only for $n=2,4$. It is nevertheless clear from Figure 14 that the usual features are present. The extent of the BK phase can be judged from the vertical rays at the Beraha numbers with $k=4,6,8$. Inside the BK phase we have a critical curve coming out of the origin with a vertical tangent and extending to the point $(q, v)=(4,-2)$. Since this is the analytical continuation of the critical curve in the ferromagnetic regime $v>0$, it must be the RG attractor governing the BK phase. We should therefore have $q_{\mathrm{c}}=4$ for the frieze lattice. 


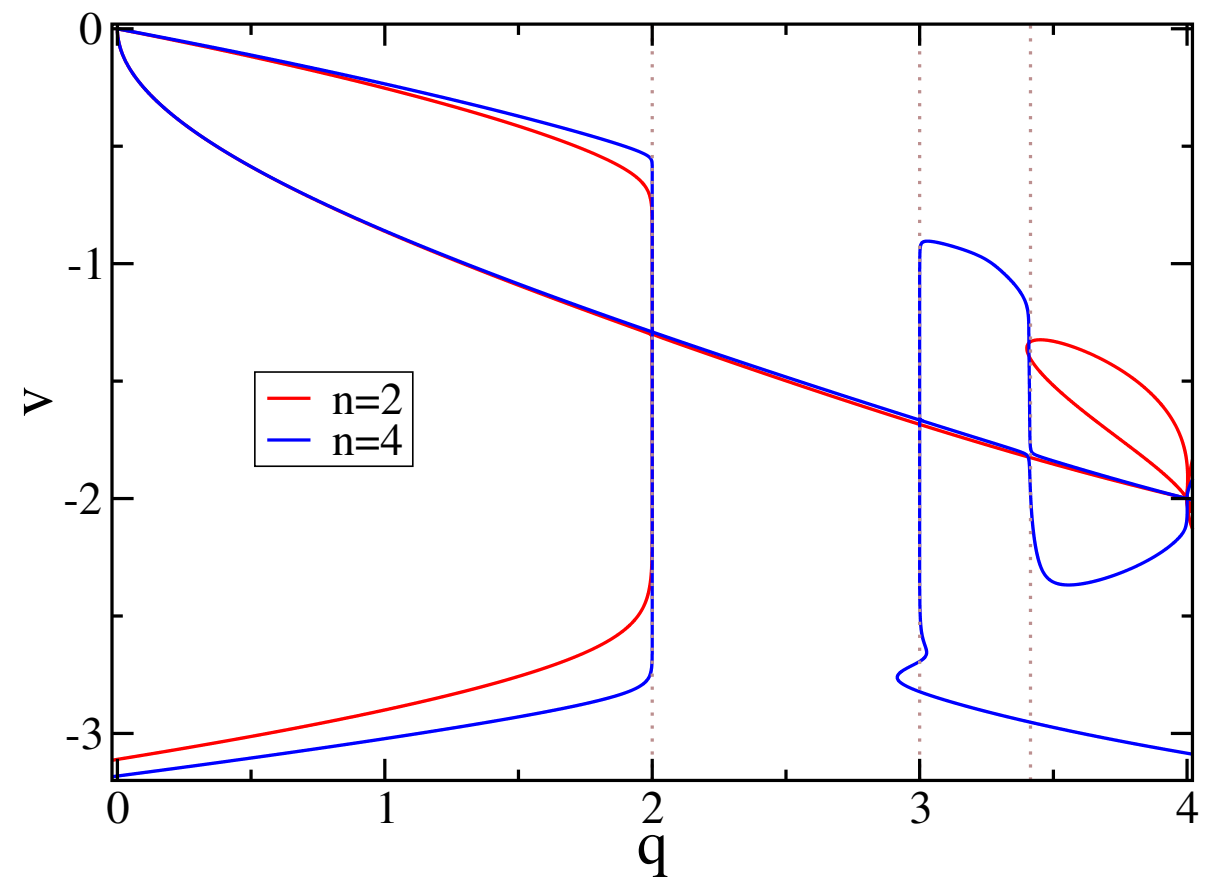

Figure 14. Roots of $P_{B}(q, v)$ for the Potts model on the frieze lattice, using $n \times n$ square bases.

The curves $v_{ \pm}(q)$ bounding the BK phase are only partially represented by the roots of the polynomials $P_{B}(q, v)$. Indeed the parts with $2<q<3$ are missing altogether. We notice however that the $n=4$ curve develops a small bulge near the bottom of the $q=3$ vertical ray, and it is conceivable that for higher $n$ this will develop into narrow "fingers" that will close the curves $v_{ \pm}(q)$ - a situation that was seen clearly in Figure 12 for the four-eight lattice.

\subsection{Three-twelve lattice $\left(3,12^{2}\right)$}

With the three-twelve lattice we are back in the category of lattices that can be represented in four-terminal form for any parity of $n$. The appropriate $\check{\mathrm{R}}$-matrix reads

$$
\check{\mathrm{R}}=\mathrm{H}_{i+1} \mathrm{~V}_{i} \mathrm{H}_{i+1} \mathrm{~V}_{i} \mathrm{E}_{i+2} \mathrm{H}_{i+1} \mathrm{~V}_{i} \mathrm{H}_{i+1} \mathrm{~V}_{i+2} \mathrm{~V}_{i} \text {. }
$$

The corresponding basis is depicted in Figure 15. It possesses 6 vertices and 9 edges per grey square, the highest numbers that we have attained among the Archimedean lattices. Accordingly we can expect the most accurate results for the critical points of this lattice (except, obviously, for the exactly solvable three-terminal cases).

Results for the percolation threshold $p_{\mathrm{c}}$ are given in Table 13, using square bases of size $n \leq 7$. Those with $n \leq 4$ have already been reported in [21] where the final estimate $p_{\mathrm{c}}=0.740420800(1)$ was proposed. The three extra data points allow us to improve considerably on this, adding four more digits to the final result reported in Table 13. We have again benefited from a very high value $w \approx 6.39$ of the parameter appearing in (30). This brings the relative precision to $1 \cdot 10^{-12}$, that is, more than four orders of 


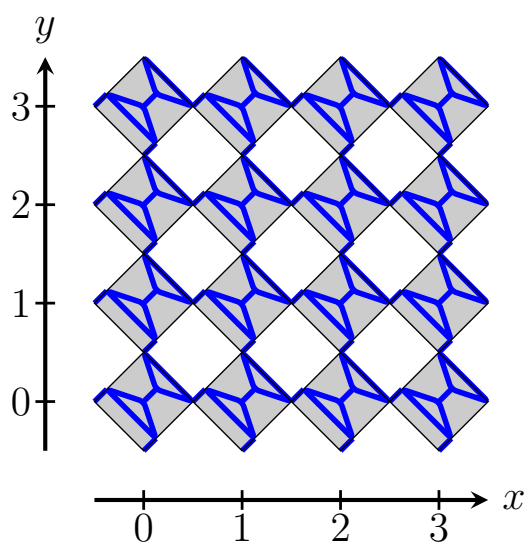

Figure 15. Four-terminal representation of the three-twelve lattice.

\begin{tabular}{l|l}
$n$ & $p_{\mathrm{c}}$ \\
\hline 1 & 0.74042331791989696781390025991096022103832189014608 \\
2 & 0.74042099242999609235990682258592153549751047668010 \\
3 & 0.74042081882197909432814081145369695940339872717580 \\
4 & 0.74042080213011204414779539731474889355503786568625 \\
5 & 0.74042079963976341893709572470832241553387184495527 \\
6 & 0.74042079909690334068260706338305717610656002517013 \\
7 & 0.74042079894276532366605540512642419991201536276834 \\
\hline$\infty$ & $0.7404207988509(8)$ \\
Ref. $[22]$ & $0.74042077(2)$
\end{tabular}

Table 13. Bond percolation threshold $p_{\mathrm{c}}$ on the three-twelve lattice.

magnitude better than the best available numerical result [22]. Note that the value of $w$ is almost coincident with that of the kagome lattice; this is presumably linked to the fact that this lattice has the same symmetry group as the three-twelve lattice.

The graph polynomials $P_{B}(q, v)$ factorise as usually in the $q=2$ Ising case. The maximum degree of the factors is $d_{\max }=4$ for $n=1,2 ; d_{\max }=8$ for $n=3$, 4 ; and $d_{\max }=16$ for $n=5$. There is precisely one of these factors, namely

$$
-8-8 v-6 v^{2}-2 v^{3}+v^{4}
$$

that possesses a positive root,

$$
v_{\mathrm{c}}=\frac{1}{2}(1+\sqrt{3}+\sqrt{2(6+5 \sqrt{3})}) \simeq 4.073446135 \cdots .
$$

Moreover, (41) is a factor in $P_{B}(q, v)$ for any size $n$. Its positive root (42) furnishes the exactly known critical point $[56,57,58]$.

The results for the critical point $v_{\mathrm{c}}$ of the Potts models with $q=3$ and $q=4$ are shown in Tables 14-15. Again we obtain very high accuracies on the final estimates. 


\begin{tabular}{l|l}
$n$ & $v_{\mathrm{c}}$ \\
\hline 1 & 5.0330225148727450936191152069088523071556984046386 \\
2 & 5.0330723130708872393918569395936083805639594860270 \\
3 & 5.0330776369208258020214559125337418019531815564544 \\
4 & 5.0330782997119322552115261369184585114757976531754 \\
5 & 5.0330784300991523816473701505184903317147518415418 \\
6 & 5.0330784662543648528166987041595243471021156868329 \\
7 & 5.0330784788156319031365051678892523632559589908672 \\
\hline$\infty$ & $5.03307848898(7)$
\end{tabular}

Table 14. Critical point $v_{\mathrm{c}}$ of the $q=3$ state Potts model on the three-twelve lattice.

\begin{tabular}{l|l}
$n$ & $v_{\mathrm{c}}$ \\
\hline 1 & 5.8573948279836477826193148233190408839405294733071 \\
2 & 5.8574980277679771830251052175741883454287604168277 \\
3 & 5.8575099292060853584152486686816804379692602098265 \\
4 & 5.8575115251380370204992586915287203141213189319694 \\
5 & 5.8575118674921102618186189385720483562901709368620 \\
6 & 5.8575119704409279852620284760092589856034565012177 \\
7 & 5.8575120089261340455809871849023068234150587067062 \\
\hline$\infty$ & $5.8575120444(3)$
\end{tabular}

Table 15. Critical point $v_{\mathrm{c}}$ of the $q=4$ state Potts model on the three-twelve lattice.

The phase diagram shown in Figure 15 has a very intricate structure, containing more features than for any of the other lattices considered so far. It was discussed in detail in [14] for $n \leq 4$, but we gain extra information - and confirmation of some of the salient features - from the $n=5$ critical polynomial $P_{B}(q, v)$ now available.

We see of course the usual vertical rays characterising the BK phase, visible at the Beraha numbers (21) with $k=4,6,8$ and building up at $k=10$ as well. The extent of the BK phase can be estimated from those rays. It seems clear that it extends out to the point $(q, v)=(4,-2)$, and thus we have $q_{\mathrm{c}}=4$ for the three-twelve lattice.

To discuss the phase diagram in some more detail, we first focus on the region $0 \leq q \lesssim 2$. We first note the formation of fingers in the $n=4,5$ curves in the range $0 \leq q \lesssim 1.2$. To the left of the lower edge of the $q=2$ vertical ray one sees the formation of a triangular-shaped enclosed region with $1.3 \lesssim q \lesssim 2$; this is visible in particular in the small wrinkle that develops in the $n=5$ curve. Moreover, there is a tiny enclosed sliver with $q \leq 2 \lesssim 2.01$ that is consistently visible for any parity of $n$. These two regions (of triangular and sliver shape) near $q=2$ are reminiscent of similar features on the kagome lattice, and we believe that they subsist in the thermodynamical limit.

For $2.8 \lesssim q \leq 4$ the situation is quite complicated. There are notably a couple of curves inside the BK phase, such that the vertical rays with $k=6,8$ are cut into three 


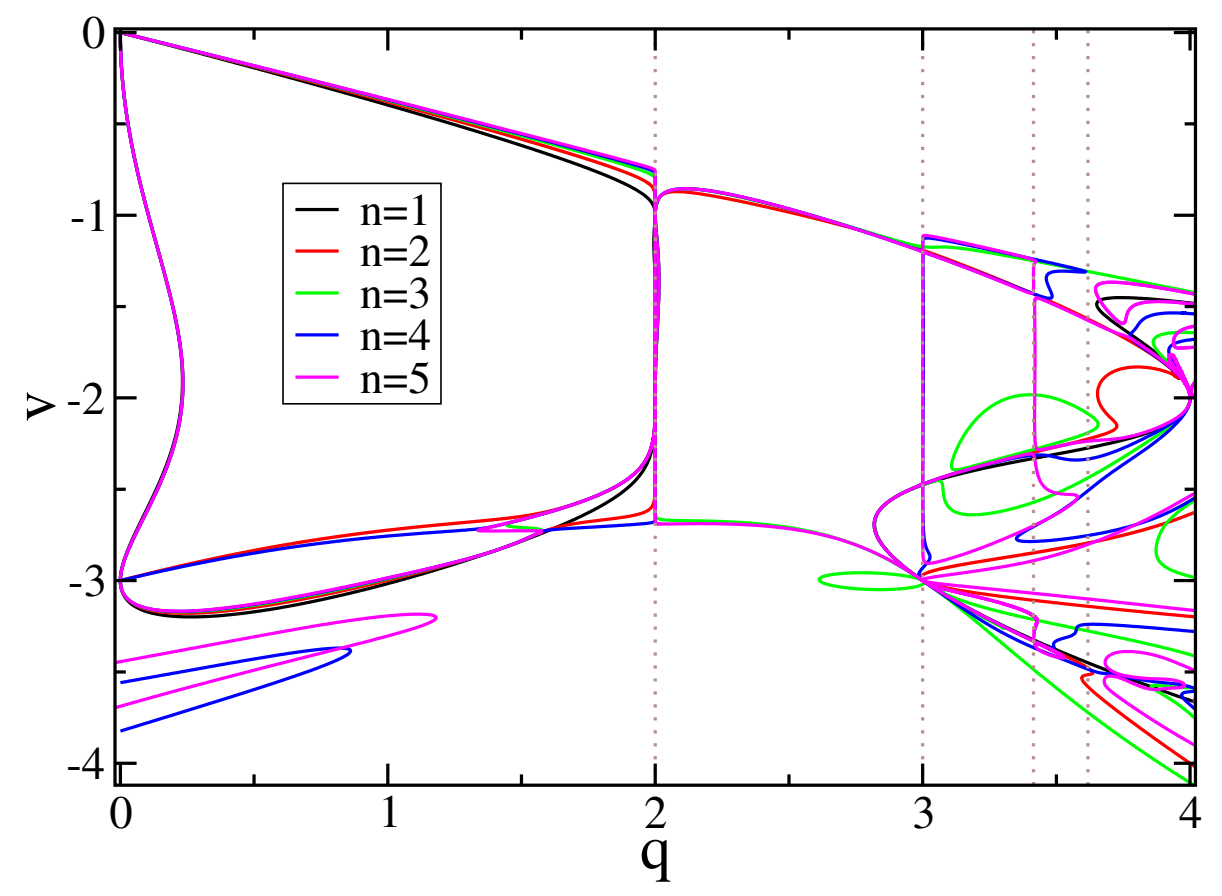

Figure 16. Roots of $P_{B}(q, v)$ for the Potts model on the three-twelve lattice, using $n \times n$ square bases.

pieces. Just above $(q, v)=(4,-2)$ one observes several extra curves developing for large $n$ which clearly tend to extend this structure to higher values of $k$. A similar situation happens near the lower boundary of the BK phase. Moreover, in the region $3 \lesssim q \lesssim 4$ and $-4 \lesssim v \lesssim-3$ many curves are building up and accumulating at $(q, v)=(3,-3)$. Clearly this latter point must have a very distinguished role in the phase diagram.

\subsection{Cross lattice $(4,6,12)$}

To cast the cross lattice in four-terminal form, four different types of grey squares are needed. The $\check{R}$-matrix takes the form

$$
\check{\mathrm{R}}_{i}= \begin{cases}\mathrm{H}_{i+1} \mathrm{~V}_{i+2} \mathrm{~V}_{i} \mathrm{H}_{i+1} \mathrm{~V}_{i+2} & \text { for } x \text { even and } y \text { even } \\ \mathrm{H}_{i+1} \mathrm{E}_{i+2} \mathrm{E}_{i} \mathrm{H}_{i+1} & \text { for } x \text { odd and } y \text { even } \\ \mathrm{H}_{i+1} \mathrm{~V}_{i+2} \mathrm{~V}_{i} \mathrm{H}_{i+1} \mathrm{\vee}_{i+2} \mathrm{~V}_{i} & \text { for } x \text { even and } y \text { odd } \\ \mathrm{H}_{i+1} \mathrm{~V}_{i+2} \mathrm{~V}_{i} \mathrm{H}_{i+1} \mathrm{~V}_{i} & \text { for } x \text { odd and } y \text { odd }\end{cases}
$$

and the resulting basis is illustrated in Figure 17. The reader is invited to check carefully from the figure that each vertex is indeed surrounded by a square, a hexagon and a dodecagon. Obviously we must require that $n$ be even. There is on average 3 vertices and $\frac{9}{2}$ edges per grey square.

The approximations to the percolation threshold $p_{\mathrm{c}}$ are shown in Table 16. Despite the parity constraint on $n$, we are still able to provide a final estimate that is more precise than those obtainable from numerical simulations [54]. 


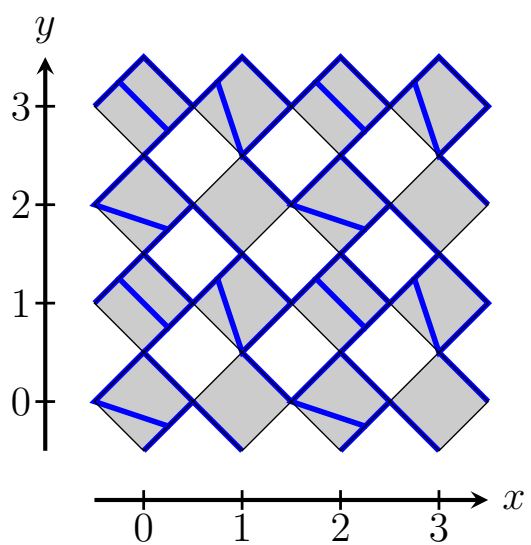

Figure 17. Four-terminal representation of the cross lattice.

\begin{tabular}{l|l}
$n$ & $p_{\mathrm{c}}$ \\
\hline 2 & 0.69377849010809934126953435770375111640557701680580 \\
4 & 0.69373853603434634159328968721278581288678588137638 \\
6 & 0.69373378891455375106650642653023991489166486978424 \\
\hline$\infty$ & $0.6937314(1)$ \\
Ref. [54] & $0.6937338(7)$
\end{tabular}

Table 16. Bond percolation threshold $p_{\mathrm{c}}$ on the cross lattice.

For $q=2$, the graph polynomials $P_{B}(q, v)$ factorise, and the maximum degree of the factors is $d_{\max }=8$ for both $n=2$ and $n=4$. The unique common factor possessing a positive root is

$$
-32-128 v-208 v^{2}-176 v^{3}-84 v^{4}-24 v^{5}+4 v^{7}+v^{8} .
$$

After some algebra this leads to the critical coupling

$$
\begin{aligned}
v_{\mathrm{c}} & =\exp \left(2 \operatorname{arctanh} \sqrt{\frac{2}{1+\sqrt{3}+\sqrt{-4+6 \sqrt{3}}}}\right)-1 \\
& \simeq 3.216563123 \cdots .
\end{aligned}
$$

in agreement with [23].

The results for the critical points $v_{\mathrm{c}}$ for the three and four-state Potts models appear in Tables $17-18$.

The phase diagram - as witnessed by the roots of the critical polynomials $P_{B}(q, v)$ combines a number of features familiar from the four-eight lattice. There is a finger in the $n=4$ curve with $0 \leq q \lesssim 1.7$ that tends to bridge the gap between the point $(q, v)=(0,-4)$ and the lower edge of the $q=2$ vertical ray. Similarly, wrinkles in both curves $(n=2,4)$ tends to delimit the BK phase from above on the interval $2<q<3$. As a result, we observe vertical rays at the Beraha numbers (21) with $k=4,6,8$. Presumably the BK phase extends out to $q_{\mathrm{c}}=4$ for this lattice. 
High-precision percolation thresholds and Potts-model critical manifolds

\begin{tabular}{l|l}
$n$ & $v_{\mathrm{c}}$ \\
\hline 2 & 3.9586332997059740720181837928768500290543859070058 \\
4 & 3.9591763776237344298683368980854435922679135046889 \\
6 & 3.9592412531275763639725384140180205407837422105870 \\
\hline$\infty$ & $3.959273(1)$
\end{tabular}

Table 17. Critical point $v_{\mathrm{c}}$ of the $q=3$ state Potts model on the cross lattice.

\begin{tabular}{l|l}
$n$ & $v_{\mathrm{c}}$ \\
\hline 2 & 4.5935119229510043784589758419016606560974164157721 \\
4 & 4.5946031686522078767110561721497673809936709287964 \\
6 & 4.5947347336947799761277325102560255207753903194732 \\
\hline$\infty$ & $4.594801(2)$
\end{tabular}

Table 18. Critical point $v_{\mathrm{c}}$ of the $q=4$ state Potts model on the cross lattice.

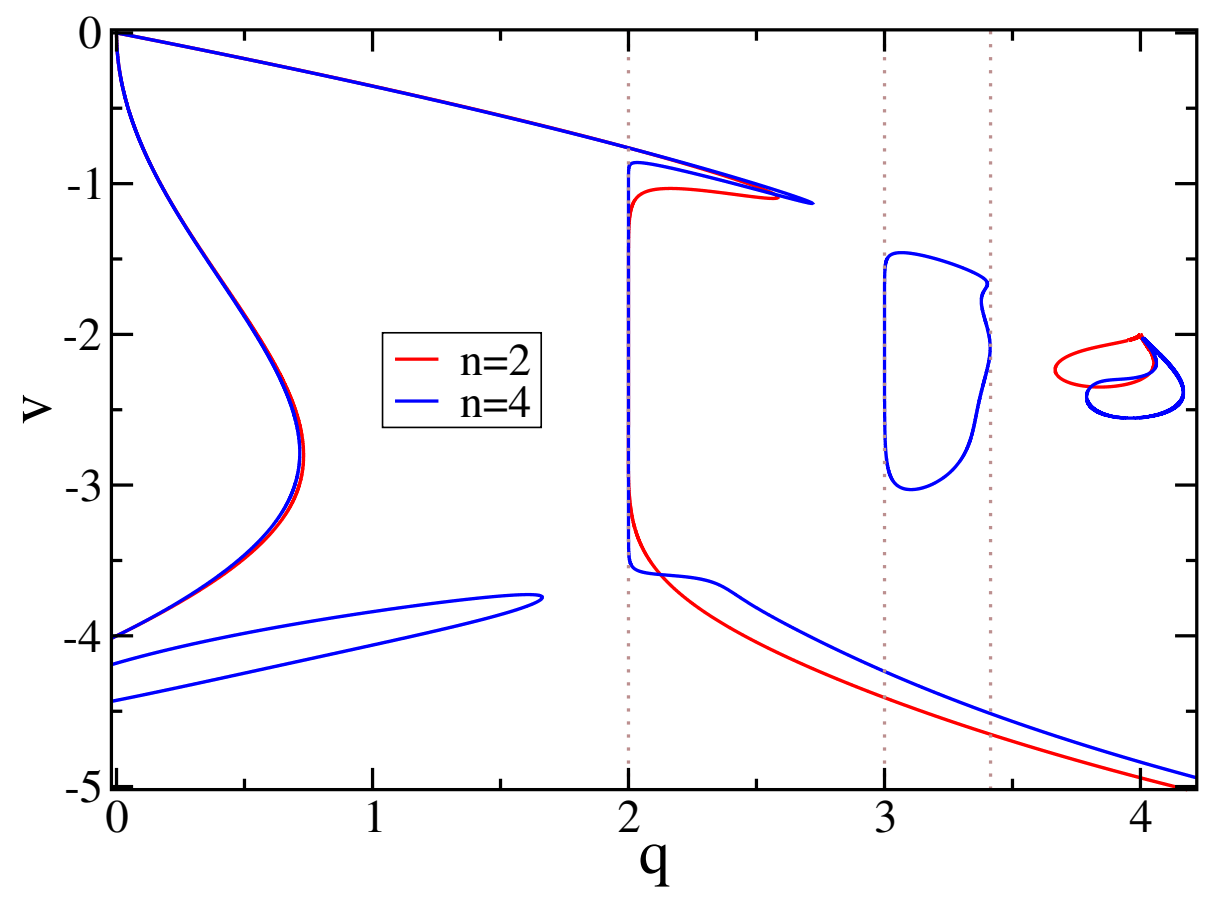

Figure 18. Roots of $P_{B}(q, v)$ for the Potts model on the cross lattice, using $n \times n$ square bases. 


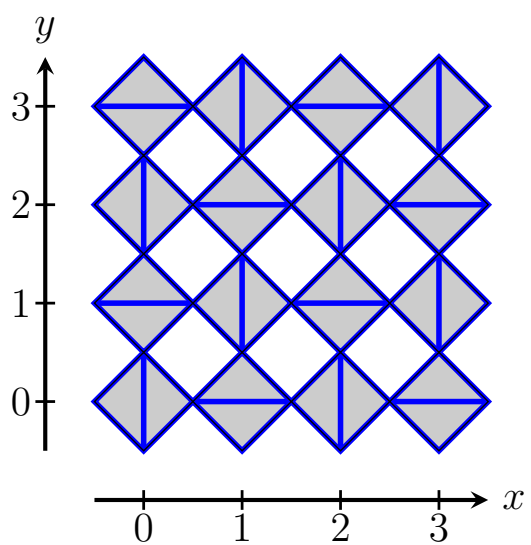

Figure 19. Four-terminal representation of the snub square lattice.

\begin{tabular}{l|l}
$n$ & $p_{\mathrm{c}}$ \\
\hline 2 & 0.41414477068891106678269962067496175696956628442687 \\
4 & 0.41413831583758918250111117204245967836572650308666 \\
6 & 0.41413794448601941204552140015728270649261591711977 \\
\hline$\infty$ & $0.4141378476(7)$ \\
Ref. [54] & $0.4141374(5)$
\end{tabular}

Table 19. Bond percolation threshold $p_{\mathrm{c}}$ on the snub square lattice.

\subsection{Snub square lattice $\left(3^{2}, 4,3,4\right)$}

The four-terminal representation of the snub square lattice is quite straightforward to obtain. The $\check{\mathrm{R}}$-matrix reads

$$
\check{\mathrm{R}}= \begin{cases}\mathrm{H}_{i+1} \mathrm{~V}_{i+2} \mathrm{H}_{i+1} \mathrm{~V}_{i} \mathrm{H}_{i+1} & \text { for } x+y \text { even } \\ \mathrm{H}_{i+1} \mathrm{~V}_{i} \mathrm{H}_{i+1} \mathrm{~V}_{i+2} \mathrm{H}_{i+1} & \text { for } x+y \text { odd }\end{cases}
$$

and Figure 19 contains a rendering of the corresponding basis. There are 2 vertices and 5 edges per grey square.

The bond percolation thresholds $p_{\mathrm{c}}$ obtained from the critical polynomials are reported in Table 19, and the corresponding results for the critical point $v_{\mathrm{c}}$ of the Potts model with $q=3$ and $q=4$ are shown in Tables 20-21.

The Ising model polynomials $P_{B}(q, v)$ with $q=2$ factorise, the maximum degree of the factors being $d_{\max }=6$ for $n=2$, and $d_{\max }=12$ for $n=4$. There is a unique common factor possessing a positive root

$$
-4-8 v-4 v^{2}+4 v^{3}+8 v^{4}+4 v^{5}+v^{6} .
$$

If we define $\omega=37+27 \sqrt{2}+3 \sqrt{315+222 \sqrt{2}}$, the critical coupling can be written

$$
v_{\mathrm{c}}=\frac{1}{3}\left(-2-2 w^{-1 / 3}+w^{1 / 3}\right) \simeq 0.980730864 \cdots
$$

and this coincides with the result of [23]. 


\begin{tabular}{l|l}
$n$ & $v_{\mathrm{c}}$ \\
\hline 2 & 1.18529390865581786407849700528021223954317049051598 \\
4 & 1.18531433669635871167720996722549305108279483104371 \\
6 & 1.18531541517566214804035962759914618124246371903443 \\
\hline$\infty$ & $1.185315678(3)$
\end{tabular}

Table 20. Critical point $v_{\mathrm{c}}$ of the $q=3$ state Potts model on the snub square lattice.

\begin{tabular}{l|l}
$n$ & $v_{\mathrm{c}}$ \\
\hline 2 & 1.35449677473518648766976021945792542217215508930616 \\
4 & 1.35453610524182819267343590204899630391908189104331 \\
6 & 1.35453810944807886217841873099783060879140651150399 \\
\hline$\infty$ & $1.354538584(6)$
\end{tabular}

Table 21. Critical point $v_{\mathrm{c}}$ of the $q=4$ state Potts model on the snub square lattice.

It remains to discuss the phase diagram, shown in Figure 20. As usual, the extent of the BK phase can be seen from the vertical rays, here appearing at Beraha numbers (21) with $k=4,6,8$. An interesting feature for this lattice is that there is a transition curve in the middle of the BK phase, reminiscent of what was found for the frieze lattice (see Figure 14). This curve emanates from the origin with vertical slope and extends out to $(q, v)=(4,-2)$. For finite $n$ there are some gaps in this curve, here visible for $n=4$ near $k=8$, but these should disappear in the thermodynamical limit. The closure of the BK phase is ensured by a wrinkle developing near the lower edge of the $q=3$ vertical ray in the $n=4$ curve, and by fingers developing at $q \approx 4$.

\subsection{Snub hexagonal lattice $\left(3^{4}, 6\right)$}

The snub hexagonal lattice is a depleted version of the triangular lattice, where $1 / 7$ of the vertices and their adjacent edges have been erased. We have constructed this lattice in two different ways.

The first construction closely parallels the one that we have used in section 4.1 for the triangular lattice. The only difference is that the $\mathrm{H}$ and $\mathrm{V}$ operators corresponding to erased edges have been replaced by just one of their two terms (the identity Id or the Temperley-Lieb generator E, as the case may be). There are then $\frac{12}{7}$ vertices and $\frac{30}{7}$ edges per grey square; note that these numbers are compatible with the coordination number being 5 .

This depletion representation is a bit easier to state than to actually write down. 


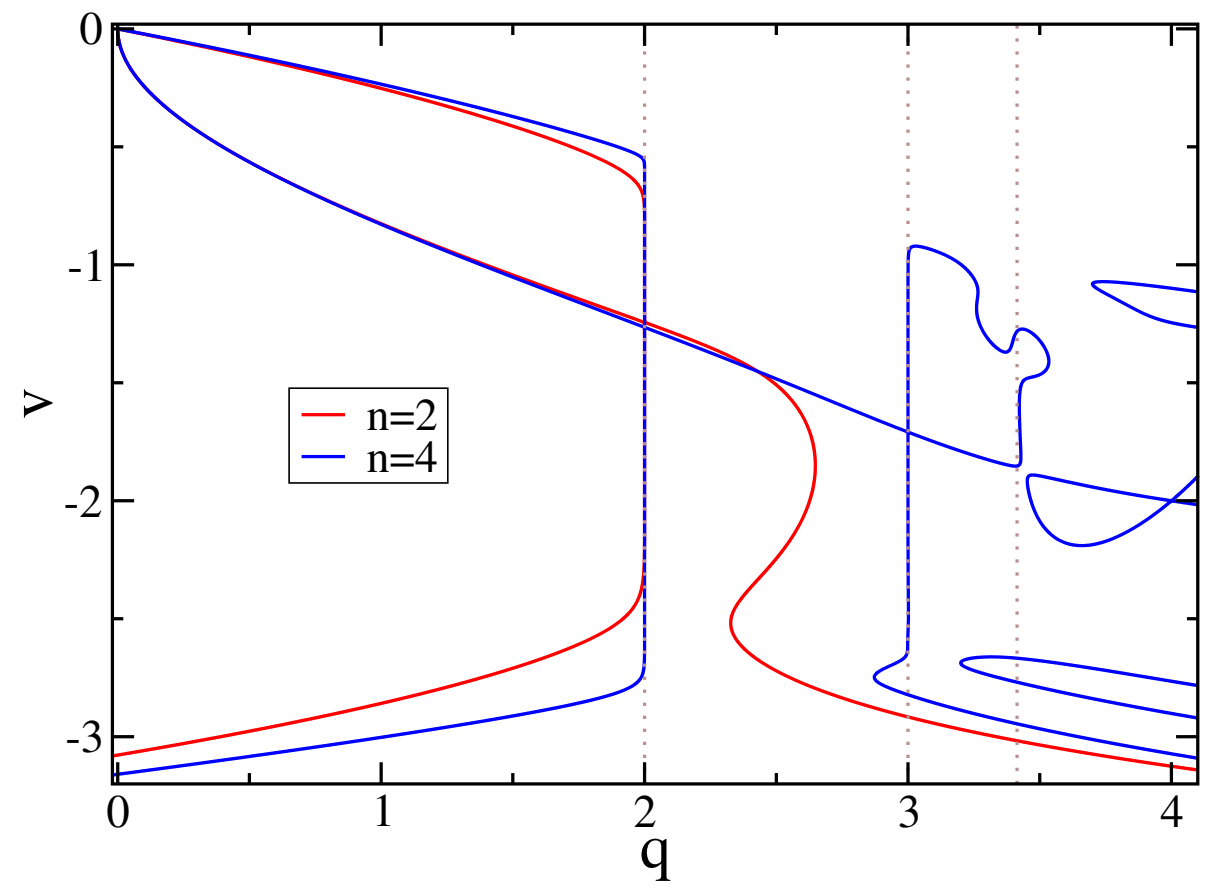

Figure 20. Roots of $P_{B}(q, v)$ for the Potts model on the snub square lattice, using $n \times n$ square bases.

But in formal terms we arrive, after drawing things carefully, at the following $\check{\text { R-matrix: }}$

$$
\check{\mathrm{R}}= \begin{cases}\mathrm{H}_{i+1} \mathrm{~V}_{i} \mathrm{H}_{i+1} \mathrm{~V}_{i+2} \mathrm{H}_{i+1} & \text { for } 5 y+x=0,2,4 \bmod 7 \\ \mathrm{H}_{i+1} \mathrm{~V}_{i} \mathrm{H}_{i+1} \mathrm{E}_{i+2} l \mathrm{~d}_{i+1} & \text { for } 5 y+x=1 \bmod 7 \\ \mathrm{Id}_{i+1} \mathrm{E}_{i} \mathrm{H}_{i+1} \mathrm{~V}_{i+2} \mathrm{H}_{i+1} & \text { for } 5 y+x=3 \bmod 7 \\ \mathrm{Id}_{i+1} \mathrm{~V}_{i} \mathrm{ld}_{i+1} \mathrm{E}_{i+2} \mathrm{H}_{i+1} & \text { for } 5 y+x=5 \bmod 7 \\ \mathrm{H}_{i+1} \mathrm{E}_{i} \mathrm{Id}_{i+1} \mathrm{~V}_{i+2} \mathrm{~d}_{i+1} & \text { for } 5 y+x=6 \bmod 7\end{cases}
$$

In addition there are horizontal diagonals in the write squares at coordinates $\left(x+\frac{1}{2}, y+\frac{1}{2}\right)$ when $5 y+x=0,1,4,5,6 \bmod 7$. Note that the first line in (49) corresponds to undepleted grey squares, i.e., it coincides with (22). Subsequent lines are obtained from the first one by depletion, i.e., replacing some of the $\mathrm{H}$ operators by the identity $\mathrm{Id}$, and some of the $\mathrm{V}$ operators by the Temperley-Lieb generator $\mathrm{E}$.

Note that this construction will just disconnect the erased spins from the remainder of the lattice. This means that when computing the critical polynomial from (49), the true $P_{B}(q, v)$ will be multiplied by a spurious factor of $q$ per erased spin. This will obviously not change the set of roots $P_{B}(q, v)=0$. We take the convention (here and elsewhere) of dividing such spurious factors out of the polynomials that are provided in electronic form in the supplementary material.

Note that (49) only makes sense when $n$ is a multiple of 7 . This means that, using this construction, the only computations that we can perform in practice is to find numerically the roots in $v$ of $P_{B}(q, v)=0$ with $n=7$. While this agrees nicely with the upper bound $n_{\max }=7$ for the feasibility of the computations, it entails two 


\begin{tabular}{l|l}
$n$ & $p_{\mathrm{c}}$ \\
\hline 2 & 0.43435240230711099756452570147287096581374932914479 \\
4 & 0.43433086696991174675056512033952861575538482310455 \\
6 & 0.43432861831549797141228558205641632097611421130662 \\
\hline 7 & 0.43432809783895257624033939574442324419941437511711 \\
\hline$\infty$ & $0.43432764(3)$ \\
Ref. [54] & $0.4343062(5)$
\end{tabular}

Table 22. Bond percolation threshold $p_{\mathrm{c}}$ on the snub hexagonal lattice.

inconveniences. First, our inability to compute the full polynomial $P_{B}(q, v)$ with $n=7$ implies that we have no access to the phase diagram in the real $(q, v)$ plane. Second, the fact that we get just one single estimate for $v_{\mathrm{c}}$ means that the only sensible proposal for the $n \rightarrow \infty$ result is the $n=7$ value itself. In particular, an error bar on this result can only be obtained by making the (a priori not unlikely) assumption that the distance between the $n=7$ value and the would-be extrapolation is comparable to that of other lattices for which we have been able to perform the extrapolation carefully.

To elaborate on this last remark, consider for instance the $n=7$ estimate for the percolation threshold shown in Table 22. Let us recall that the relative deviation between the $n=7$ estimate and the $n \rightarrow \infty$ extrapolated value is $2 \cdot 10^{-9}$ for the kagome lattice (see Table 4 ) and $1 \cdot 10^{-10}$ for the three-twelve lattice (see Table 13). However, these two lattices benefited from very high values $(w \approx 6.36$ and $w \approx 6.39$ respectively) of the correction-to-scaling exponent in (30). For the four-eight lattice (see Table 7) we found a smaller value $w \approx 4.28$ and accordingly the relative precision of the $n=7$ estimate comes out as $8 \cdot 10^{-8}$. Recall also that the basis for the kagome and four-eight (resp. three-twelve) lattices has 6 (resp. 9) edges per grey square. From the number of edges in the basis, we thus have no a priori reason to believe that the convergence properties of the snub hexagonal lattice (whose basis has $\frac{30}{7} \simeq 4.3$ edges per grey square) would be significantly worse than any of those lattices. However, since the value of $w$ for the snub hexagonal lattice is presently unknown, we could conservatively assume that the $n=7$ data point has a relative precision of only $8 \cdot 10^{-7}$, and use this assumption to provide a tentative error bar:

$$
p_{\mathrm{c}}=0.4343281(4) \quad \quad \text { (tentative result) }
$$

Noting that even this conservative estimate is in significant disagreement with the numerical result of Parviainen [54] (see Table 22) we therefore turn to a different way on constructing the snub hexagonal lattice.

The second construction is based on the four-terminal representation shown in Figure 21. $\dagger$ It generalises the general four-terminal setup of Figure 2 by stretching the white squares vertically, so that they are now hexagons. To make these hexagons more visible in the figure we have shaded them alternately using white and pink colours,

$\dagger$ The author is grateful to Chris Scullard for pointing out this representation. 


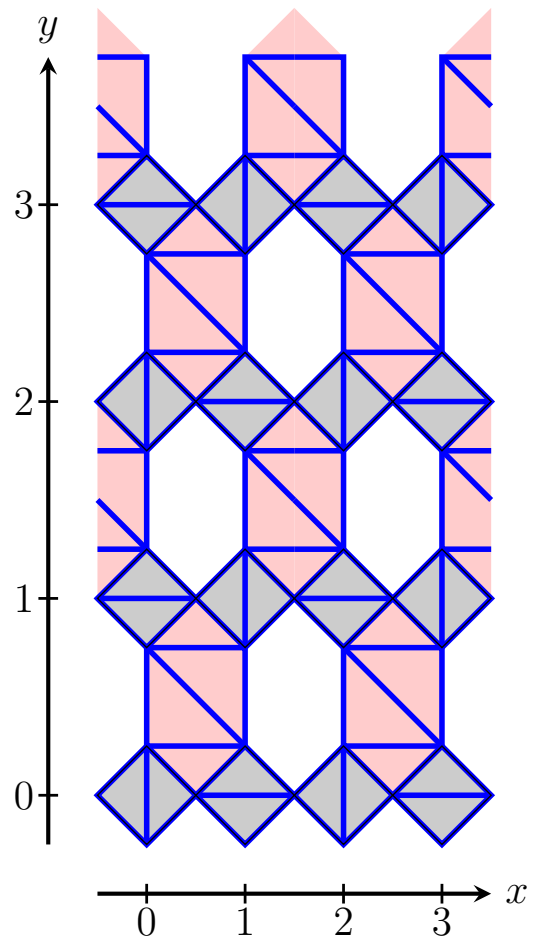

Figure 21. Four-terminal representation of the snub hexagonal lattice.

but we shall still refer to them as "white hexagons". The R-matrix describing the grey squares is exactly the same as that of the snub square lattice, see Eq. (46). The essential characteristics of the white hexagons is that-just like the white squares used previously - the lattice structure inside them can be constructed without the use of auxiliary spaces. The corresponding operator (the analogue of $\check{\mathrm{R}}_{i}$ ) reads

$$
\mathrm{O}_{i} \equiv \mathrm{H}_{i+1} \mathrm{~V}_{i+2} \mathrm{H}_{i+1} \mathrm{~V}_{i} \mathrm{H}_{i+1} \text {. }
$$

After each even row (i.e., $y=\frac{1}{2} \bmod 2$ ) the operator $\mathrm{O}_{i}$ acts at positions $i=0 \bmod 4$, and after each odd row (i.e., $y=\frac{3}{2} \bmod 2$ ) it acts at $i=2 \bmod 4$. Note that $\mathcal{O}_{i}$ and $\mathcal{O}_{i+4}$ commute, since each operator acts on precisely four adjacent strands, and so we do not need to specify the order of factors in these products. Moreover, since $\mathcal{O}_{i}$ now contains operators of the type $\mathrm{V}$ that propagate the system upwards, it is crucial for this representation that every other white hexagon be empty. This is indeed the case, as shown by the alternating white and pink shadings of the "white" hexagons.

The representation of Figure 21 makes sense for even $n$. It contains 3 vertices and $\frac{15}{2}$ edges per grey square. This is a significant improvement over the first construction described in this subsection. We note that the basis with $n=2$ coincides with that used in [20, Figure21a] in which only percolation (not the general $q$-state Potts model) was studied. We have checked that our result for $P_{B}(1, v)$ with $n=2$ is proportional to [20, Eq. (27)] after the usual change of variables, $p=v /(1+v)$.

The percolation thresholds $p_{\mathrm{c}}$ using both the first and the second constructions are shown in Table 22. We have separated the results using the two different constructions 


\begin{tabular}{l|l}
$n$ & $v_{\mathrm{c}}$ \\
\hline 2 & 1.25822914824213089627783168669624673560080841355215 \\
4 & 1.25831385989581118739654002272081781643725758811430 \\
6 & 1.25832226936396475004073969036992612800230543696904 \\
\hline 7 & 1.25832368118331690296415028885813907747723413111257 \\
\hline$\infty$ & $1.25832577(4)$
\end{tabular}

Table 23. Critical point $v_{\mathrm{c}}$ of the $q=3$ state Potts model on the snub hexagonal lattice.

\begin{tabular}{l|l}
$n$ & $v_{\mathrm{c}}$ \\
\hline 2 & 1.42904479893946258801335842598986168793665913956403 \\
4 & 1.42921237939109852302475951646616375686887686926371 \\
6 & 1.42922900017262995076127341190422753009029194456598 \\
\hline 7 & 1.42923133461518622283008319875598743145981594637169 \\
\hline$\infty$ & $1.42923591(9)$
\end{tabular}

Table 24. Critical point $v_{\mathrm{c}}$ of the $q=4$ state Potts model on the snub hexagonal lattice.

by a horizontal line in this and the following two tables. Extrapolating the results with $n=2,4,6$ leads to the estimate for the $n \rightarrow \infty$ limit shown in Table 22. This is in agreement with the tentative result (50), but is obviously more precise since we now take advantage of a well-controlled extrapolation procedure. Note that we find $w \approx 2.94$, significantly lower than for the four-eight lattice, so our precautions in arriving at (50) were justified. The final result of Table 22 enhances our disagreement with [54] for this lattice.

For the Ising model $(q=2)$ the polynomials $P_{B}(q, v)$ factorise as usual. The degree of the largest factor is $d_{\max }=8$ for $n=2$ and $d_{\max }=12$ for $n=4$. The recurrent factor that leads to a positive real root is

$$
-8-8 v-4 v^{2}+4 v^{3}+8 v^{4}+4 v^{5}+v^{6} .
$$

If we define $\omega=37+27 \sqrt{3}+3 \sqrt{6(66+37 \sqrt{3})}$, the critical coupling can be written in the same way as (48), viz.

$$
v_{\mathrm{c}}=\frac{1}{3}\left(-2-2 w^{-1 / 3}+w^{1 / 3}\right) \simeq 1.050155297 \cdots,
$$

and this coincides with the result of [23]. Using the first construction, we have performed a 50-digit numerical computation to verify that this number is also a root of the $n=7$ polynomial. This agreement provides compelling evidence that both constructions of $P_{B}(q, v)$ are correct.

Our results for the critical point $v_{\mathrm{c}}$ in the $q=3$ and $q=4$ state Potts models are shown in Tables 23-24. Also in those cases have we based the $n \rightarrow \infty$ extrapolations 


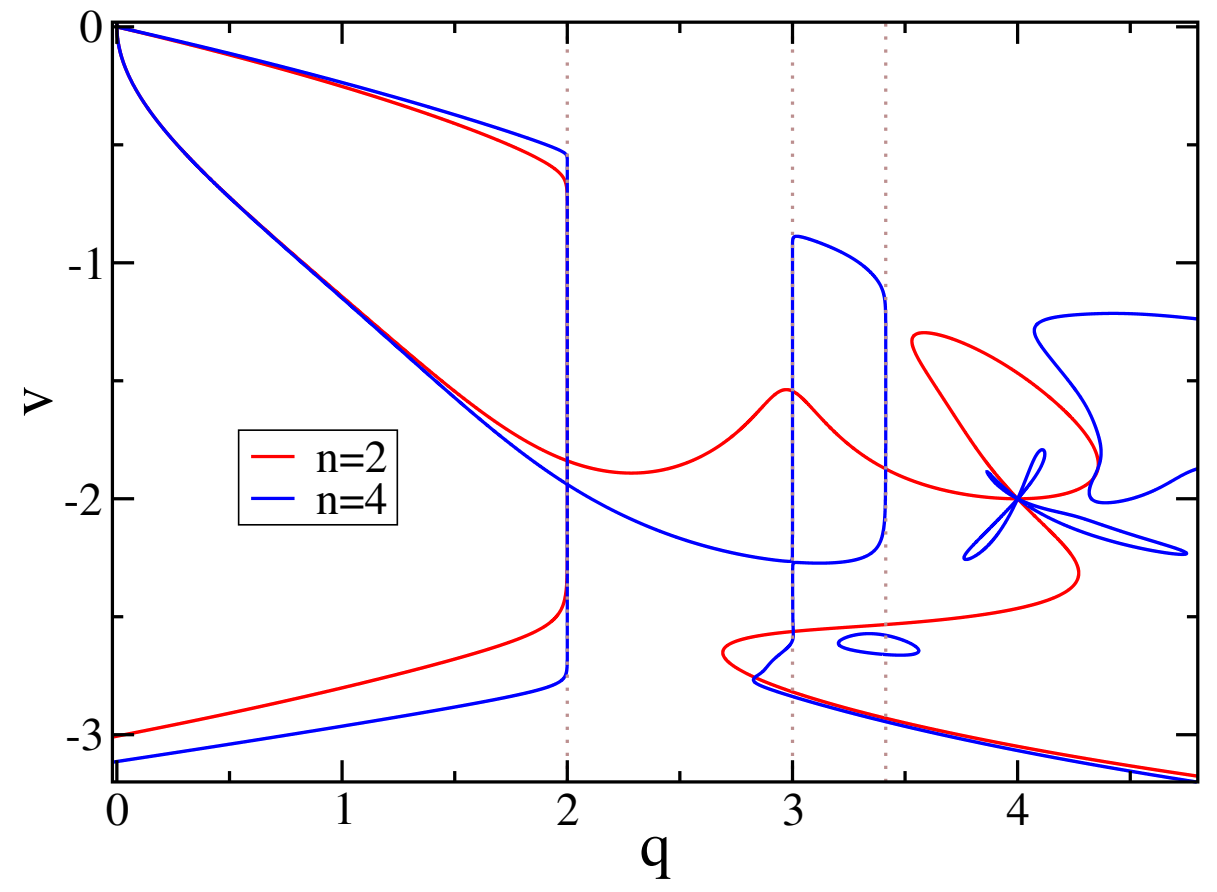

Figure 22. Roots of $P_{B}(q, v)$ for the Potts model on the snub hexagonal lattice, using $n \times n$ square bases.

on the results coming from the second construction (with $n=2,4,6$ ). Using the same kind of reasoning as discussed above for percolation, we observe that the $n=7$ results are compatible with these extrapolated values.

The phase diagram of the snub hexagonal lattice is shown in Figure 22. The extent of the BK phase can be estimated from the vertical rays at $q=B_{k}$ with $k=4,6,8$. Its upper and lower boundaries $v_{ \pm}(q)$ are interrupted by a hiatus for $2<q<3$ with these bases, but the wrinkle developing near the bottom of the $q=3$ ray in the $n=4$ curve indicates that this gap may be partly filled in at larger sizes. The curve coming out of $(q, v)=(0,0)$ with infinite slope cuts through the BK phase. We also note a rather rich structure near $q=4$ where a flower-like structure with four petals grows out of the point $(q, v)=(4,-2)$. There are thus 4 (resp. 8) branches of the curve coming out that point for $n=2$ (resp. $n=4$ ), and this number might well continue to grow for larger $n$.

\subsection{Ruby lattice $(3,4,6,4)$}

The ruby lattice is most simply treated by considering the corresponding dual lattice. A four-terminal representation of the basis of the ruby dual lattice is shown in Figure 23. The reader can check that it is indeed a quadrangulation, with each of the quadrangles being surrounded by vertices of degrees $3,4,6$ and 4 . The $\check{R}$-matrix reads

$$
\check{\mathrm{R}}= \begin{cases}\mathrm{V}_{i} \mathrm{H}_{i+1} \mathrm{~V}_{i+2} \mathrm{~V}_{i} \mathrm{H}_{i+1} & \text { for } x+y \text { even } \\ \mathrm{H}_{i+1} \mathrm{~V}_{i+2} \mathrm{~V}_{i} \mathrm{H}_{i+1} \mathrm{~V}_{i+2} & \text { for } x+y \text { odd }\end{cases}
$$




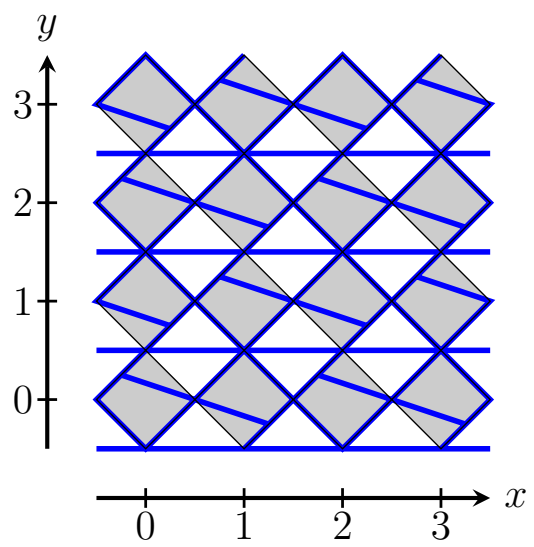

Figure 23. Four-terminal representation of the ruby dual lattice.

\begin{tabular}{l|l}
$n$ & $p_{\mathrm{c}}$ \\
\hline 2 & 0.52483166874192588456166387740108217826717357914594 \\
4 & 0.52483306819526131752968049973689841123051783280705 \\
6 & 0.52483166919384159966725735129503659944406282355902 \\
\hline$\infty$ & $0.5248311(1)$ \\
Ref. [54] & $0.5248326(5)$
\end{tabular}

Table 25. Bond percolation threshold $p_{\mathrm{c}}$ on the ruby lattice.

and there are horizontal diagonals on all the white squares. Clearly this representation of the ruby dual lattice requires $n$ to be even. There are 3 faces (corresponding to vertices of the ruby lattice itself) and 6 edges per grey square.

The graph polynomials $P_{B}(q, v)$ for the ruby lattice (and their specialisation $P_{B}(p)$ to the percolation case) are then found from those of the dual by a simple duality transformation. Details on duality will be deferred to section 5 .

Bond percolation thresholds obtained from $P_{B}(p)$ are reported in Table 25 . Uncharacteristically for the graph polynomial approach the convergence to the thermodynamical limit is here seen to be non-monotonic. This is presumably due to the smallest result $(n=2)$ straying away from the general trend. Combined with the parity constraint on $n$ this negatively impacts the precision of the extrapolated threshold $p_{\mathrm{c}}$ for this lattice.

For the Ising case $(q=2)$, the graph polynomials $P_{B}(q, v)$ factorise once again, and the maximum degree of the factors is $d_{\max }=6$ for $n=2$, and $d_{\max }=10$ for $n=4$. There is a unique common factor possessing a positive root:

$$
-8-8 v+4 v^{3}+v^{4} .
$$

The critical coupling reads

$$
v_{\mathrm{c}}=\sqrt{3+2 \sqrt{3}}-1 \simeq 1.542459756 \cdots
$$


High-precision percolation thresholds and Potts-model critical manifolds

\begin{tabular}{l|l}
$n$ & $v_{\mathrm{c}}$ \\
\hline 2 & 1.87394547154498212745580696195918007388348035664800 \\
4 & 1.87391585478227655442007815269131135001811822203444 \\
6 & 1.87392197066479367079676783453126504408425961116844 \\
\hline$\infty$ & $1.8739245(6)$
\end{tabular}

Table 26. Critical point $v_{\mathrm{c}}$ of the $q=3$ state Potts model on the ruby lattice.

\begin{tabular}{l|l}
$n$ & $v_{\mathrm{c}}$ \\
\hline 2 & 2.15108293916710713480143126975686391827111703551182 \\
4 & 2.15100732273079876867673763885401274828091470382521 \\
6 & 2.15101806147233059726929249029635229313711666783623 \\
\hline$\infty$ & $2.1510225(9)$
\end{tabular}

Table 27. Critical point $v_{\mathrm{c}}$ of the $q=4$ state Potts model on the ruby lattice.

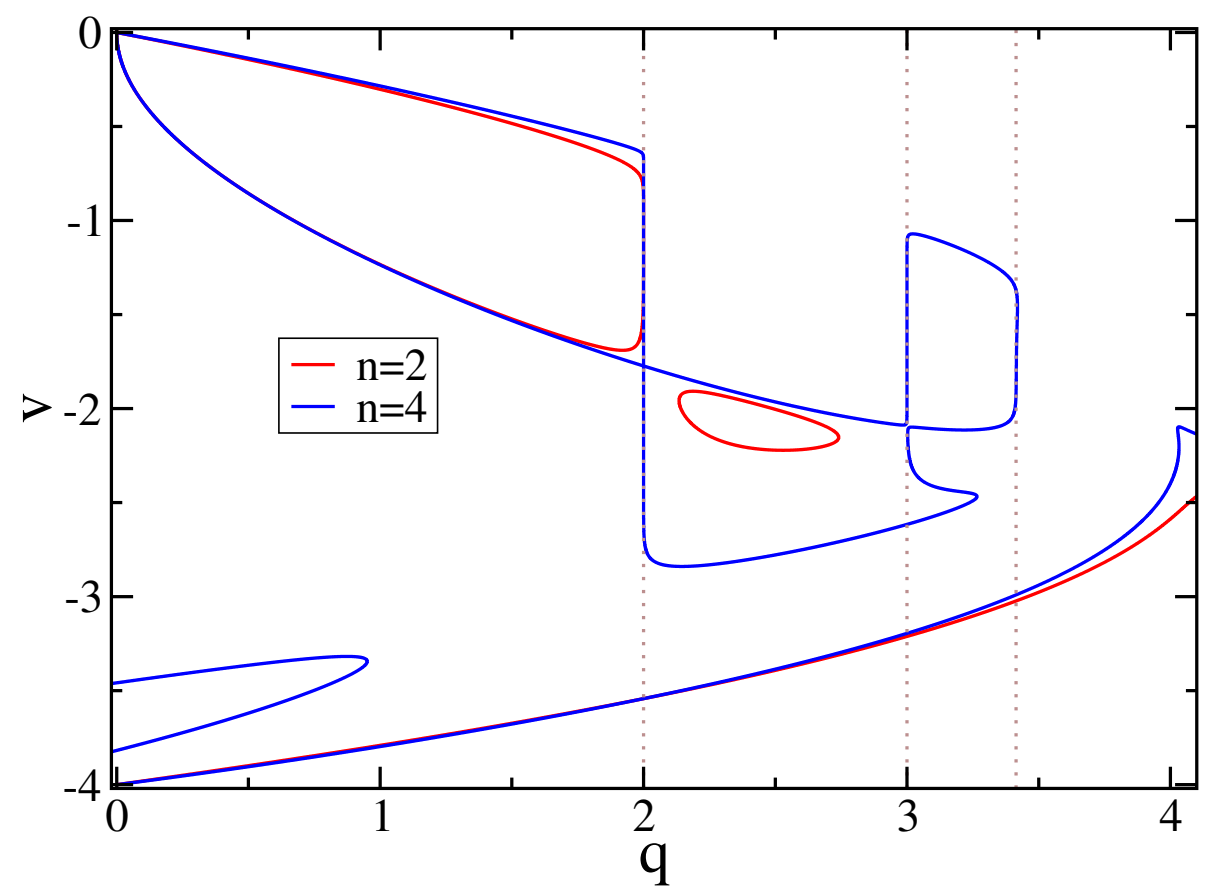

Figure 24. Roots of $P_{B}(q, v)$ for the Potts model on the ruby lattice, using $n \times n$ square bases.

in agreement with [23]. Rather curiously, this is identical to the result (32) found for the Ising model on the kagome lattice.

The critical points for the $q=3$ and $q=4$ models are shown in Tables 26-27. As for percolation the convergence is non-monotonic, preventing us from attaining the usual precision in the final results. 
The phase diagram for the ruby lattice is shown in Figure 24. The extent of the BK phase can be judged from the vertical rays at the Beraha numbers (21) with $k=4,6,8$. The upper and lower boundaries of the BK phase are only partially brought out by the largest size $n=4$. In particular, the upper boundary between $q=2$ and $q=3$ is still missing. The lower boundary between $q=0$ and $q=2$ is partially provided by the finger protruding from $q=0$, and one notes the formation of a wrinkle to the right of $q=3$. Interestingly there is another curve, emanating from $(q, v)=(0,-4)$ that lies below the lower boundary of the BK phase. This curve extends towards the point $(q, v)=(4,-2)$, and presumably the boundaries of the BK phase will also join at that point. We therefore conjecture that $q_{\mathrm{c}}=4$ for this lattice.

\subsection{Factorisable cases}

A remarkable property of the critical polynomial $P_{B}(q, v)$ is that it usually factorises in exactly solvable cases $[13,14]$. This is true in particular for the exactly solvable lattices (square, triangular and hexagonal). This is also true for the Ising model $(q=2)$ on any of the lattices considered. Note however that some cases also exist where $P_{B}(q, v)$ fails to factorise, even though the model is known to be exactly solvable. This is so in particular for the zero-temperature antiferromagnetic three-state model, $(q, v)=(3,-1)$, on the kagome lattice and for the entire chromatic line, $v=-1$, on the triangular lattice. Indeed, Baxter has shown that the latter model is integrable [45, 46], and the former model (three-state kagome) is equivalent to a special case of the latter (four-state triangular) by means of an exact mapping [53].

Conversely, it is compelling to consider any systematic factorisation of $P_{B}(q, v)$ as evidence that the model may be exactly solvable (by "systematic factorisation" we mean a factorisation that occurs for any value of the size $n$ ).

In this section we examine exhaustively the issue of factorisation for all the Archimedian lattices. We consider the following cases: Integer $q=0,1,2,3,4$, the chromatic polynomial $v=-1$, the flow polynomial $v=-q[59,60]$, and the limit $(q, v) \rightarrow(0,0)$ with fixed $w=v / q$ that correspond to spanning forests [61] with weight $1 / w$ per component tree.

For $q=0, P_{B}(q, v)$ factorises for all the lattices, producing a root $v=0$. This is consistent with the observation that for all the lattices there is a branch of the critical curve going through the point $(q, v)=(0,0)$. This describes the problem of spanning trees, which can in turn be related to free (symplectic) fermions with central charge $c=-2[62,61,63]$. Moreover, the triangular, kagome and three-twelve lattices have a root $(q, v)=(0,-3)$. And the square, four-eight, cross and ruby lattice have a root $(q, v)=(0,-4)$. By duality, the models with $(q, v)=\left(0, v_{\mathrm{c}}\right)$ are equivalent [61] to models of spanning forests on the corresponding dual lattice with weight $v_{\mathrm{c}}$ per component tree. These models can in turn be formulated as interacting fermionic theories [62, 63], and we conjecture that they are in fact exactly solvable. It follows, still by duality, that the spanning tree problem factorises on the square lattice with $w=-1 / 4$ and on the 
hexagonal lattice with $w=-1 / 3$. We find moreover that spanning trees on the cross lattice factorise with $w=-1 / 3$.

The case of the snub square and snub hexagonal lattices is interesting. For $q=0$, the critical polynomials of both these lattices shed the small factor $8+5 v+v^{2}$, and we conjecture that the corresponding roots $v=(-5 \pm i \sqrt{7}) / 2$ are loci of exact solvability.

The Ising case $(q=2)$ has been extensively discussed in the preceding sections. Cases where $P_{B}(2, v)$ has a negative integer root in $v$ occur only for $v=-1$ (the chromatic polynomial) and $v=-2$ (the flow polynomial). More precisely, $v=-1$ factorises for the triangular, kagome, frieze, three-twelve, snub square, snub hexagonal and ruby lattices. And $v=-2$ factorises for the hexagonal, four-eight, frieze, threetwelve, cross, snub square and snub hexagonal lattices.

For $q=3, P_{B}(q, v)$ has a root at $v=-3$ for the four-eight and three-twelve lattices. Note that these are three-flow problems or, equivalently, three-colouring problems of the corresponding dual lattices.

Finally, for $q=4$ there is a root at $v=-2$ for all lattices except the kagome lattice. Motivated by this, and by the phase diagrams reported in the preceding sections, we conjecture that the BK phase extends to $(q, v)=(4,-2)$ for all the Archimedian lattices, except the kagome lattice.

\section{Results on dual lattices}

The Potts model partition function admits the duality transformation $[6,59]$

$$
v \rightarrow v^{*}:=q / v .
$$

It is a consequence of (6) that the same is true for the graph polynomial $P_{B}(q, v)$. To see this, note that the configurations contributing to $Z_{2 \mathrm{D}}$ are in bijection with those contributing to $Z_{0 \mathrm{D}}^{*}$ on the dual lattice, and vice versa. It follows that

$$
P_{B^{*}}\left(q, v^{*}\right)=\frac{\left(v^{*}\right)^{|E|}}{q^{|V|}} P_{B}\left(q, q / v^{*}\right)
$$

is the graph polynomial on the dual lattice, corresponding to the dual basis $B^{*}$. Here $|V|$ and $|E|$ denote respectively the number of vertices and edges in the basis $B$.

All the results given in section 4 can therefore be applied to the dual Archimedian lattices (Laves lattices) as well, simply by making the change of variables (58). Note that in the case of the ruby lattice (section 4.10) we have already anticipated on this relation, because it is easier to represent the ruby dual lattice in the required four-terminal form than the ruby lattice itself. A few duality arguments were also used in section 4.11.

\section{Results on medial lattices}

We have also computed the graph polynomial $P_{B}(q, v)$ for all the medials of the Archimedian lattices. Medial lattices were defined and discussed in section 2. We recall that the square lattice is its own medial, $\mathcal{M}\left(4^{4}\right)=\left(4^{4}\right)$, the triangular and hexagonal 


\begin{tabular}{l|lll} 
Lattice & Vertices & Edges & Parity of $n$ \\
\hline Four-eight medial & 6 & 12 & Any \\
Frieze medial & $\frac{5}{2}$ & 5 & Any $^{\dagger}$ \\
Three-twelve medial & 9 & 18 & Any \\
Cross medial & $\frac{9}{2}$ & 9 & Even \\
Snub square medial & $\frac{5}{2}$ & 5 & Even \\
Snub hexagonal medial & $\frac{15}{7}$ & $\frac{30}{7}$ & $0 \bmod 7$ \\
& $\frac{5}{2}$ & 5 & $0 \bmod 3^{\dagger}$ \\
Ruby medial & $\frac{3}{2}$ & 3 & $0 \bmod 4$ \\
\hline
\end{tabular}

Table 28. Number of vertices and edges per grey square (cf. Figure 2) for the medials of Archimedean lattices studied here, using square bases of size $n \times n$ grey squares ( ${ }^{\dagger}$ or rectangular bases of size $n \times 2 n$ ). In addition we state any parity constraint on $n$. For the snub hexagonal medial lattices two different constructions are provided.

lattices have the same medial which is the kagome lattice, $\mathcal{M}\left(3^{6}\right)=\mathcal{M}\left(6^{3}\right)=(3,6,3,6)$, and the medial of the kagome lattice is the ruby lattice, $\mathcal{M}(3,6,3,6)=(3,4,6,4)$.

So we shall consider in the following subsections the remaining seven medial lattices. Some of these require specific tricks - which might be of independent interest - such as avoiding the introduction of intermediate points by acting in each grey square with a generic Temperley-Lieb operator, and deleting and contracting some of the edges by formally setting the coupling constants to $x=0$ or $x=\infty$.

The degree of the critical polynomials $P_{B}(q, v)$ is $k_{q} n^{2}$ in the $q$-variable and $k_{v} n^{2}$ in the $v$-variable, where $k_{q}$ and $k_{v}$ are tabulated in the second and third columns of Table 28. We have $k_{v}=2 k_{q}$ throughout, since all the medial lattices are four-regular (i.e., all their vertices are of degree 4 ).

The polynomials that we have obtained explicitly are available in electronic form as supplementary material to this paper. $\dagger$

\subsection{Four-eight medial lattice $\mathcal{M}\left(4,8^{2}\right)$}

A four-terminal representation of the four-eight medial lattice is shown in Figure 25. At first sight it does not appear feasible to write the corresponding $\stackrel{\mathrm{R}}{\mathrm{n}}$-matrix in the usual form, viz., as a product of the single-edge operators $\mathrm{V}_{i}, \mathrm{H}_{i+1}$ and $\mathrm{V}_{i+2}$ (or, more generally, the Temperley-Lieb generators $\mathrm{E}_{i}, \mathrm{E}_{i+1}$ and $\mathrm{E}_{i+2}$ ) acting within the unit cell shown in Figure 4. The problem is that we need an intermediate point in the spin representation, or two intermediate strands in the loop representation that would be situated between those labeled $i+1$ and $i+2$ in Figure 4 . These intermediate strands can however be eliminated once the grey square is completed, and they are not needed for connecting among themselves the grey squares of which the lattice consists.

$\dagger$ In the form of a text file PB.m that can be processed by Mathematica or any other symbolic computer algebra software. 


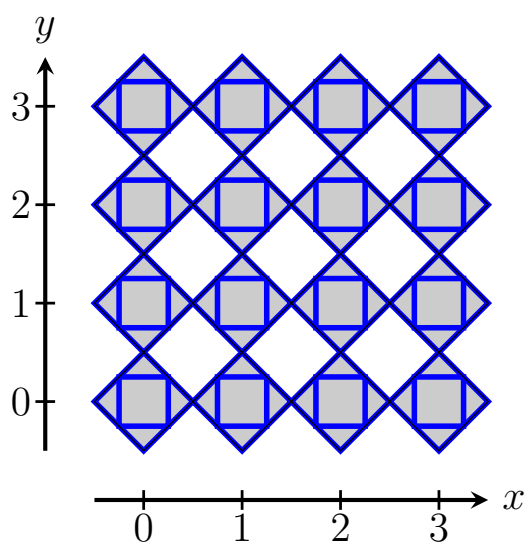

Figure 25. Four-terminal representation of the four-eight medial lattice.

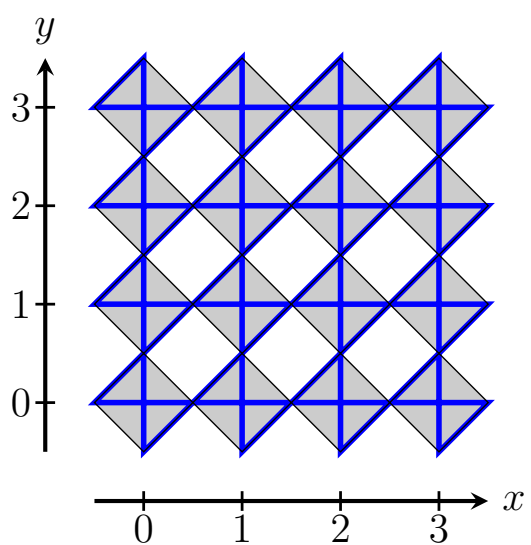

Figure 26. Alternative four-terminal representation of the kagome lattice.

To avoid dealing with intermediate points, the most efficient solution is to write down directly the entire $\check{R}$-matrix that propagates strands $i, i+1, i+2, i+3$ into $i^{\prime},(i+1)^{\prime},(i+2)^{\prime},(i+3)^{\prime}$. Recall that a single-edge operator, such as $\mathrm{V}_{i}$, consists of two terms $\left(\operatorname{Id}_{i}\right.$ and $\left.\mathrm{E}_{i}\right)$, since there are Cat $(2)=2$ possible planarity-respecting pairings of the four points $i, i+1, i^{\prime},(i+1)^{\prime}$. Similarly, the entire $\check{R}$-matrix contains in general fourteen terms, corresponding to the Cat(4) $=14$ pairings of eight points that respect planarity. We have therefore written a version of the algorithm in which a lattice is specified by supplying the fourteen terms of a generic R-matrix, each of which are polynomials in $n_{\text {loop }}$ and $x$ with integer coefficients. Further remarks on this version can be found in section 3.6.

In the case at hand we remark that $\check{\mathrm{R}}_{i}=\left(\mathrm{B}_{i}\right)^{2}$, where $\mathrm{B}_{i}$ denotes the bow tie operator, first discussed for the special case of percolation in [21, section 3.2] and subsequently generalised to the Potts model in [14, section 3.2]. Squaring the explicit expression [14, Eq. (27)] we therefore obtain the fourteen polynomials defining $\check{\mathrm{R}}_{i}$, each of which contains up to a maximum of 10 monomials $x^{a} n_{\text {loop }}^{b}$. 


\begin{tabular}{l|l}
$n$ & $p_{\mathrm{c}}$ \\
\hline 1 & 0.54490357617280539732583696992078943358822055517888 \\
2 & 0.54482333432473326673827916421900410932649253360415 \\
3 & 0.54480395308638647849435301431084308963838444393987 \\
4 & 0.54479979248458363100469869489549952372478071895348 \\
5 & 0.54479869412491430661835928269715088171915677271830 \\
6 & 0.54479832681576092745539465910054157272244608840374 \\
7 & 0.54479817718177358100731028878250145618621980837755 \\
\hline$\infty$ & $0.544798017(4)$ \\
Ref. $[64,65]$ & $0.5447979(3)$ \\
&
\end{tabular}

Table 29. Bond percolation threshold $p_{\mathrm{c}}$ on the four-eight medial lattice.

To test the general algorithm we have also investigated the case where each $\mathrm{R}$-matrix is a single bow tie operator, $\check{\mathrm{R}}_{i}=\mathrm{B}_{i}$. This can be represented as in Figure 26. Obviously this is just a rotated version of the four-terminal representation of the kagome lattice shown in Figure 9. We have validated the "generic R-matrix" algorithm by verifying that in this case it gives the very same critical polynomials $P_{B}(q, v)$ as those obtained in section 4.3. The choice of transfer direction made in section 4.3 is slightly more efficient for dealing with the kagome lattice, since $\check{\mathrm{R}}_{i}$ involves the application of six operators with each two terms $(6 \times 2=12)$ rather than a single operator with fourteen terms. Moreover, the approach with two terms per operator involves only very simple coefficients $(1$ or $x)$.

Returning to the four-eight medial lattice, we remark that in Figure 25 there are 6 vertices and 12 edges per grey square (see Table 28). The approximations to the bond percolation threshold $p_{c}$ obtained from the unique positive root of $P_{B}(p)$ are shown in Table 29.

For the Ising model $(q=2)$ the polynomials $P_{B}(q, v)$ always factorise. The maximum degree of the factors is $d_{\max }=8$ for $n=1,2, d_{\max }=16$ for $n=3$, and $d_{\max }=12$ for $n=4$. One of these factors, namely

$$
-64-128 v-160 v^{2}-96 v^{3}-8 v^{4}+32 v^{5}+24 v^{6}+8 v^{7}+v^{8},
$$

occurs systematically for any $n$. By the change of variables $v=-1+\sqrt{y}$ this simplifies to

$$
-23-20 y-18 y^{2}-4 y^{3}+y^{4}
$$

The unique positive root is

$$
v_{\mathrm{c}}=-1+(1+\sqrt{2}+\sqrt{10+8 \sqrt{2}})^{1 / 2} \simeq 1.651582692 \cdots .
$$

We expect this to be the exact critical point, although we are not aware of any exact solution of the Ising model on the four-eight medial lattice. 


\begin{tabular}{l|l}
$n$ & $v_{\mathrm{c}}$ \\
\hline 1 & 1.9922041076260751644586139440972263436652635871358 \\
2 & 1.9926404728708502640118089145818512148977796047886 \\
3 & 1.9927384787472335781145657627229386691955129596069 \\
4 & 1.9927586076549435383693884954495153641240804012262 \\
5 & 1.9927637904129256320901803185635371343359435001717 \\
6 & 1.9927654949220578625901153681198029149913267082580 \\
7 & 1.9927661807712696317912790872926681763051395367055 \\
\hline$\infty$ & $1.99276689(2)$
\end{tabular}

Table 30. Critical point $v_{\mathrm{c}}$ of the $q=3$ state Potts model on the four-eight medial lattice.

\begin{tabular}{l|l}
$n$ & $v_{\mathrm{c}}$ \\
\hline 1 & 2.2755212087361303992802668673758851902898410610266 \\
2 & 2.2763771760824237004043542724461772715320090614142 \\
3 & 2.2765630817464703256746144539003180310447940465275 \\
4 & 2.2766003034722388602617749274377947499719604673753 \\
5 & 2.2766097352625615580128595130617901964243752604729 \\
6 & 2.2766128023187296094751641612708914426382050828433 \\
7 & 2.2766140251425216149873240698999853443282508981560 \\
\hline$\infty$ & $2.27661527(5)$
\end{tabular}

Table 31. Critical point $v_{\mathrm{c}}$ of the $q=4$ state Potts model on the four-eight medial lattice.

Moreover, the critical points for the $q=3$ and $q=4$ state Potts models are given in Tables 30-31.

The phase diagram for the four-eight medial lattice is rather simple; see Figure 27. There is a clear vertical ray at the Beraha number (21) with $k=4$; and possibly the $n=3$ polynomial also indicates that a ray will emerge at $k=6$, although the $n=4$ result fails to confirm this. It seems likely that the BK phase will extend to the arc near $q \approx 3.3$, although its upper and lower boundaries are not visible on the interval $2<q<3$ with these critical polynomials. In any case, the absence of vertical rays for $k>6$ is a clear sign that $q_{\mathrm{c}}<4$ for this lattice. We also note that all curves go through the point $(q, v)=(0,-3)$ exactly.

\subsection{Frieze medial lattice $\mathcal{M}\left(3^{3}, 4^{2}\right)$}

For the frieze medial lattice we can use the four-terminal representation depicted in Figure 28. It consists of alternating rows of grey squares of the types used in the kagome and square lattices. Therefore, the $\check{\mathrm{R}}$-matrix is given by (29) on even rows, and by (25) on odd rows. It is thus convenient to use rectangular bases of size $n \times 2 n$ grey 


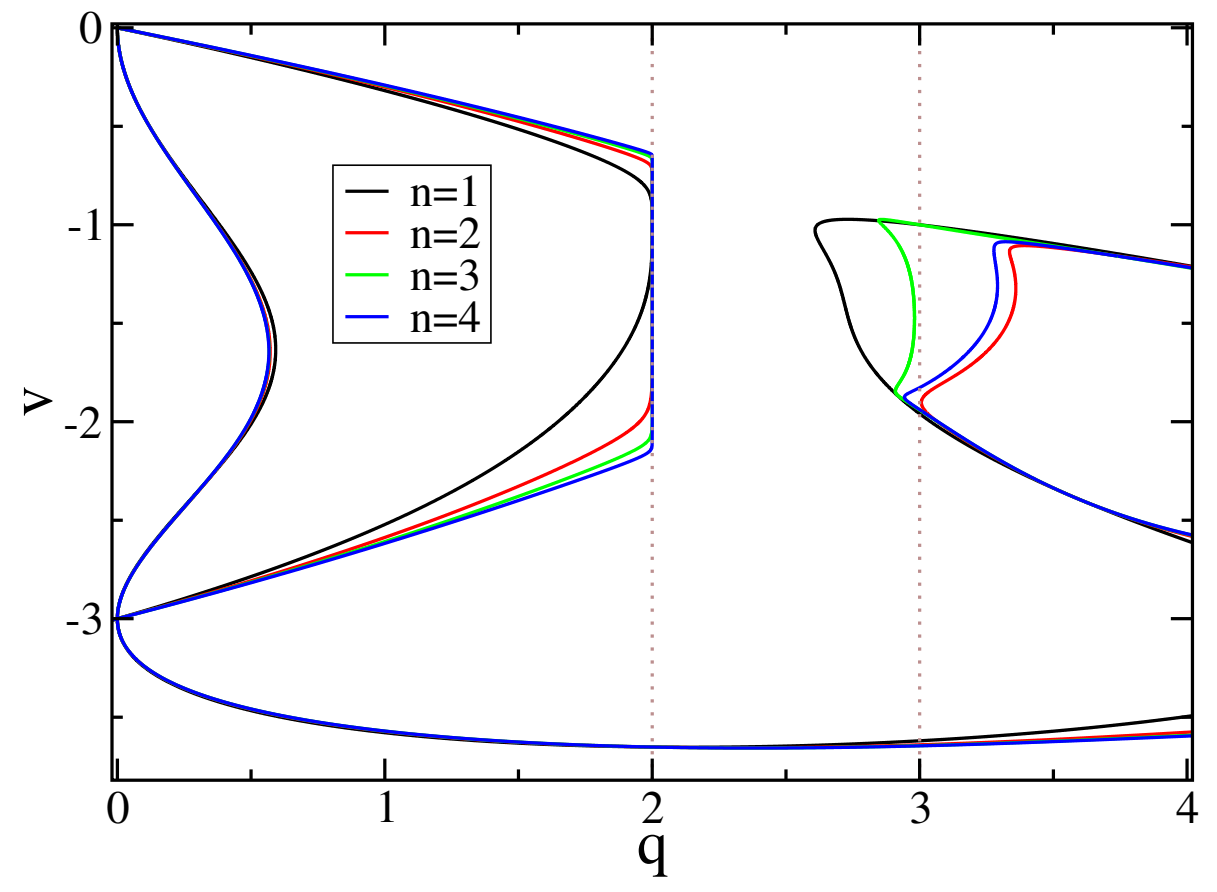

Figure 27. Roots of $P_{B}(q, v)$ for the Potts model on the four-eight medial lattice, using $n \times n$ square bases.

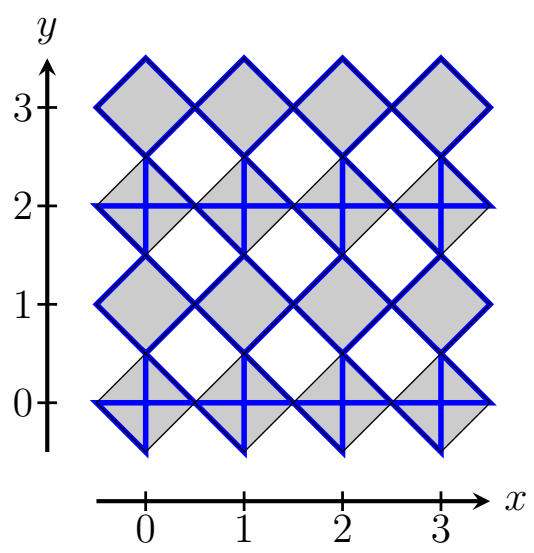

Figure 28. Four-terminal representation of the frieze medial lattice.

squares, for any parity of $n$. There are $\frac{5}{2}$ vertices and 5 edges per grey square.

The corresponding bond percolation thresholds $p_{c}$ are shown in Table 32 .

When $q=2$ we obtain as usual a factorisation of $P_{B}(q, v)$. The maximum degree of the factors is $d_{\max }=8$ for $n=1,2, d_{\max }=16$ for $n=3,4$, and $d_{\max }=32$ for $n=5$. The factor relevant for determining the critical point simplifies upon setting $v=-1+\sqrt{y}$ and becomes

$$
1-20 y-10 y^{2}-4 y^{3}+y^{4} .
$$

Its physically relevant solution has an expression in terms of cube roots, which is however 


\begin{tabular}{l|l}
$n$ & $p_{\mathrm{c}}$ \\
\hline 1 & 0.51252671239052872734284920277093949452459399814557 \\
2 & 0.51252555030832881329338864757758450264150947803966 \\
3 & 0.51252505197721914539499792825297076237457413214236 \\
4 & 0.51252476509531218613179917728236141170706073572125 \\
5 & 0.51252466184456998405019250652673909947600021592946 \\
6 & 0.51252462541470314484904781495993557924808658518665 \\
7 & 0.51252461131296365065118129973998555670342068841944 \\
\hline$\infty$ & $0.5125245984(9)$
\end{tabular}

Table 32. Bond percolation threshold $p_{\mathrm{c}}$ on the frieze medial lattice.

\begin{tabular}{l|l}
$n$ & $v_{\mathrm{c}}$ \\
\hline 1 & 1.80713435658088600169174675586705957329793973933928 \\
2 & 1.80714787637307386116716755890184038031063366743447 \\
3 & 1.80715275038905311055716653443917611922430053521537 \\
4 & 1.80715499141679873814200897705704030717174184979977 \\
5 & 1.80715574557129979201879822669803898126598246764060 \\
6 & 1.80715600281645714745051778969055570176041887478573 \\
7 & 1.80715610046331273129256920306307290435340513061371 \\
\hline$\infty$ & $1.807156187(2)$
\end{tabular}

Table 33. Critical point $v_{\mathrm{c}}$ of the $q=3$ state Potts model on the frieze medial lattice.

\begin{tabular}{l|l}
$n$ & $v_{\mathrm{c}}$ \\
\hline 1 & 2.08199719749764750866564040310148994909037162782967 \\
2 & 2.08202742833616593271859950614838779880367025625026 \\
3 & 2.08203865409278753166570381108592447912012172754718 \\
4 & 2.08204360154127867025506347751737245230091088735469 \\
5 & 2.08204524006200808869976649798749897664563660237247 \\
6 & 2.08204579575822659373529481034339920792570530958012 \\
7 & 2.08204600668989993066296166093996927319153647466394 \\
\hline$\infty$ & $2.08204619(3)$
\end{tabular}

Table 34. Critical point $v_{\mathrm{c}}$ of the $q=4$ state Potts model on the frieze medial lattice.

to lengthy to be reported here. It corresponds to $v_{\mathrm{c}} \simeq 1.479990057 \cdots$ in the original variable.

The critical points $v_{\mathrm{c}}$ for the $q=3$ and $q=4$ state Potts models appear in Tables 33-34.

The phase diagram of the frieze medial lattice, shown in Figure 29, is exceedingly complicated, and arguably even more complicated than that of the three-twelve lattice 


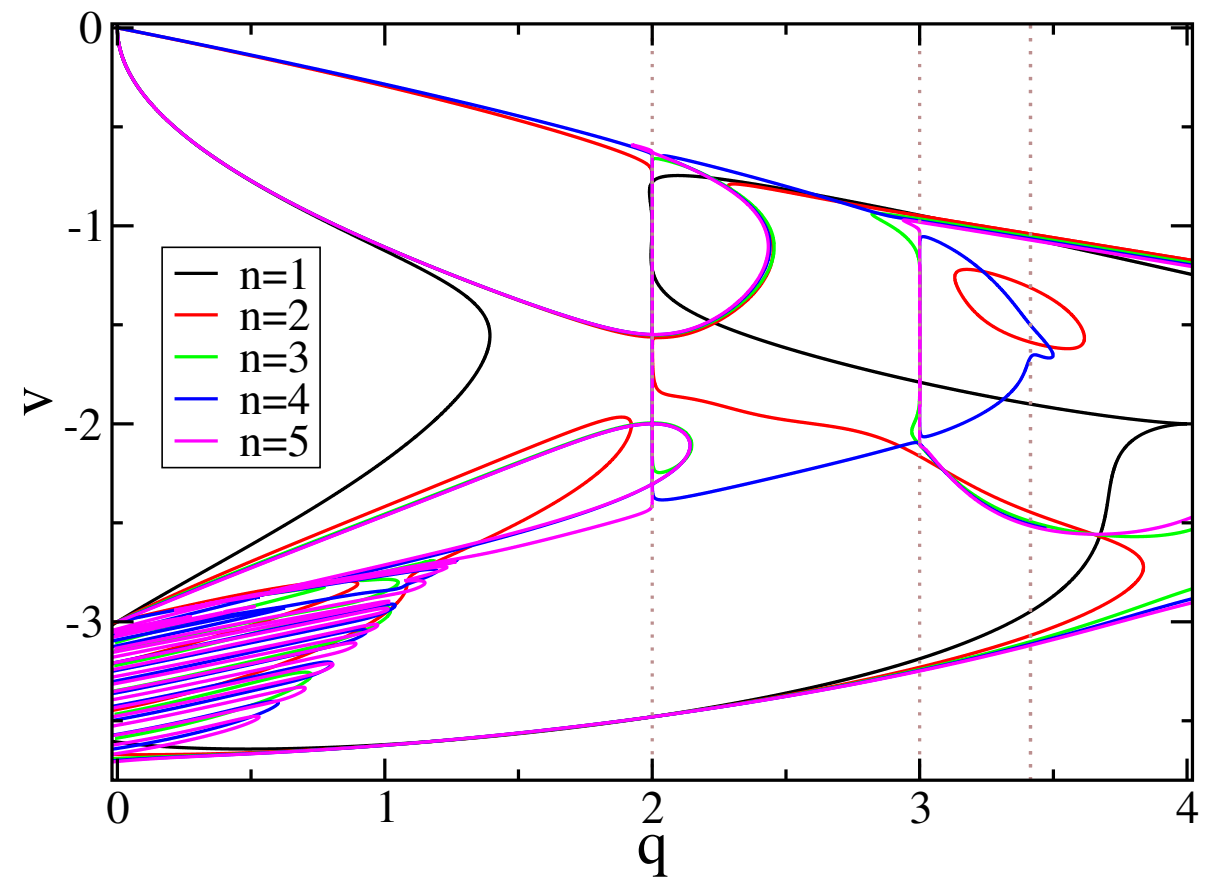

Figure 29. Roots of $P_{B}(q, v)$ for the Potts model on the frieze medial lattice, using $n \times 2 n$ rectangular bases.

(see Figure 16). The upper limit $v_{+}(q)$ of the BK phase contains an almost straight part from the origin to the neighbourhood of $(q, v)=(3,-1)$. The almost straight continuation to higher $q$ cannot be the upper limit of the BK phase, though, since it is not adjacent to the characteristic vertical rays. Rather, the continuation of $v_{+}(q)$ must be provided by the $n=4$ arc that bends around at $(q, v) \approx(3.5,-1.6)$. For $n=2$ this arc is prefigured by a bubble that is not connected to the rest of the curves. It seems likely that all even $n$ will participate to this part of the phase diagram. Note in particular that the $n=4$ arc has a small wrinkle at $q \simeq B_{8}$ which would most likely turn into a vertical ray for higher (even) $n$. One can therefore believe that $q_{\mathrm{c}}>B_{8}$ for this lattice, but it is yet unclear whether $q_{\mathrm{c}}$ might be as large as 4 . After the $n=4$ arc bends around, it traces out the lower limit $v_{-}(q)$ of the BK phase that continues to the point $(q, v)=(0,-3)$ through which all curves pass exactly. The extent of the BK phase can be judged from the vertical rays at $q=B_{k}$ with $k=4,6$ (and maybe 8 as just mentioned).

Two further branches of the curve come out of $(q, v)=(0,-3)$ and trace out a finger that extends a little further than the vertical ray at $q=2$. There is a similar, broader finger coming out of $(q, v)=(0,0)$, whose upper side coincides with $v_{+}(q)$.

Apart from these features, there is almost horizontal branch coming out of $(q, v) \approx$ $(0,-3.7)$ and extending towards large $q$. Similarly, the bottom and top of the vertical ray at $q=3$ connect to branches that extend towards large $q$.

A close-up of the region near $(q, v)=(0,-3)$ is shown in Figure 30. Throughout this region the curves become increasingly dense as $n$ increases. Counting the number of 


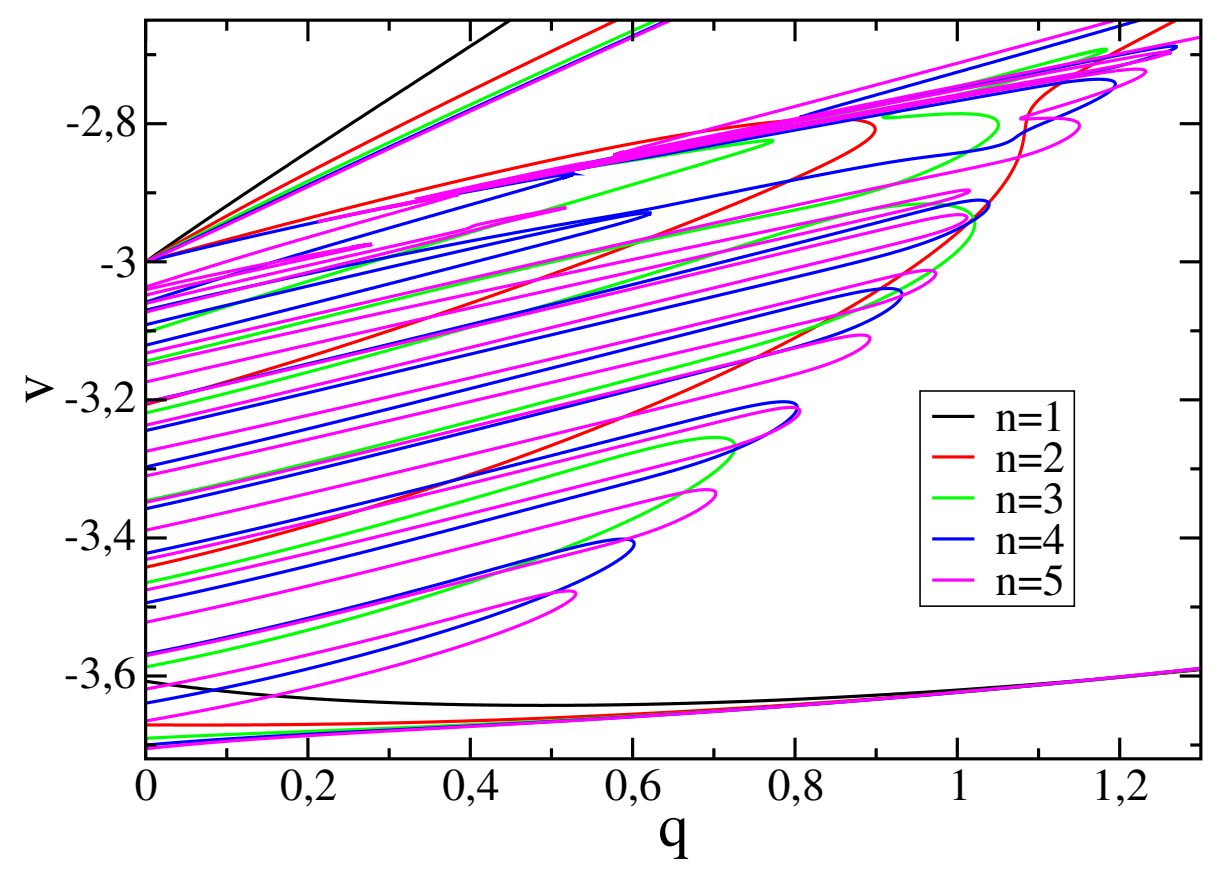

Figure 30. Close-up on a region of Figure 29.

fingers emanating from the $v$-axis in the range $-3.7<v<3$ gives compelling evidence for the conjecture that the curves will, in fact, become space-filling in this region when $n \rightarrow \infty$. This is a novel feature, not seen in the phase diagrams for any of the other lattices. It is very reminiscent of a recent study of the phase diagram of the Potts model on a family of non-planar graphs, called the generalised Petersen graphs [66], where a number of "critical regions" (marked by a $\star$ in [66, Figure 2]) were identified throughout which the two dominant eigenvalues of the transfer matrix are exactly degenerate in norm. Such critical regions also exist in two dimensions, and in particular for the $q$ state Potts model on the triangular lattice close to the point $(q, v)=(0,-3)$, as well as for $q>4[47]$.

This capability of the $P_{B}(q, v)=0$ curves to be space-filling might also offer a new interpretation of the thin fingers emanating from the $v$-axis in many of the preceding figures (see, e.g., Figure 16). Might it be that these fingers will also become more numerous and tend to fill out space for larger (i.e., not accessible in this paper) values of $n$ ? We leave this question for future investigations.

\subsection{Three-twelve medial lattice $\mathcal{M}\left(3,12^{2}\right)$}

A four-terminal representation of the three-twelve medial lattice is shown in Figure 31. This lattice is also known as the $2 \times 2$ kagome subnet [65], since it can be obtained by replacing each of the triangles of the kagome lattice by an equilateral made of $2 \times 2=4$ triangles. In our representation there are 9 vertices and 18 edges per grey square - the highest numbers for any lattice considered in this paper. This means that the basis for 


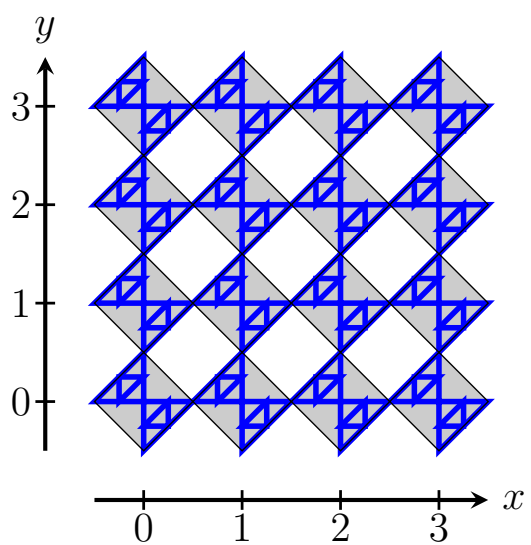

Figure 31. Four-terminal representation of the three-twelve medial lattice.

the $n=7$ numerical computation encompasses $18 \times 7^{2}=882$ edges, as mentioned in the abstract.

The $\check{R}$-matrix can only be computed in the sparse-matrix factorisation scheme if one inserts intermediate points. In this case, three such points (or six loop strands) are needed. Just like in section 6.1, it is therefore advantageous to use the "generic R-matrix" version of the algorithm. The fourteen weights can be readily computed separately, from a transfer matrix that builds a single grey square, using time slices of width five points (or ten loop strands). Alternatively, this may be done by hand defining a transfer process where time flows in the North-West (rather than the usual NorthEast) direction, and using the fact that $\check{\mathrm{R}}_{i}=\tilde{\mathrm{S}}_{i} \mathrm{~S}_{i}$, where $\mathrm{S}_{i}$ denotes the $2 \times 2$ subnet operator, and $\tilde{S}_{i}$ is its time-reflected counterpart. Either way, we obtain the fourteen polynomials defining $\check{\mathrm{R}}_{i}$, each of which contains up to a maximum of 30 monomials $x^{a} n_{\text {loop }}^{b}$. These are obviously too lengthy to be reproduced here, but are available upon request from the author.

The bond percolation thresholds $p_{c}$ are shown in Table 35. As for the three-twelve lattice itself, we obtain a very substantial improvement on the precision of the existing results, here by more than four orders of magnitude.

For the Ising model $(q=2)$ we have the usual factorisation of $P_{B}(q, v)$. The maximum degree of the factors is $d_{\max }=8$ for $n=1,2, d_{\max }=16$ for $n=3,4$. The relevant factor for determining the critical point has degree 8 in the $v$-variable, but changing variables through $v=-1+\sqrt{y}$ we find the simper polynomial

$$
-83-32 y-6 y^{2}-8 y^{3}+y^{4} .
$$

The unique root that corresponds to a positive value of $v$ reads

$$
v_{\mathrm{c}}=-1+(2+\sqrt{3}+\sqrt{2(6+5 \sqrt{3})})^{1 / 2} \simeq 2.024382957 \cdots .
$$

Once again, we expect this to be the exact critical point, although we are not aware of any exact solution of the Ising model on the three-twelve medial lattice. 


\begin{tabular}{l|l}
$n$ & $p_{\mathrm{c}}$ \\
\hline 1 & 0.60087024823863130165397946806873749105812018535152 \\
2 & 0.60086257369370252495888862646970191568249607022723 \\
3 & 0.60086202873714154724433811970387661781266525679198 \\
4 & 0.60086197705173039926972062364498505003371758838282 \\
5 & 0.60086196938791403077982454158989347857185134707268 \\
6 & 0.60086196771854575664675151979737081972939591265171 \\
7 & 0.60086196724363425411431529583585404028066985304771 \\
\hline$\infty$ & $0.600861966960(2)$ \\
Ref. $[22]$ & $0.60086193(3)$
\end{tabular}

Table 35. Bond percolation threshold $p_{\mathrm{c}}$ on the three-twelve medial lattice.

\begin{tabular}{l|l}
$n$ & $v_{\mathrm{c}}$ \\
\hline 1 & 2.4051388771937835004651461060627027673916305236110 \\
2 & 2.4052102689837864921984641492409181211413650400579 \\
3 & 2.4052176562543970511035491279473526360116379644946 \\
4 & 2.4052185619190028113565896890585979366394650085930 \\
5 & 2.4052187382745471274900934254447806792951661256278 \\
6 & 2.4052187868761485331493140625659769323839252659120 \\
7 & 2.4052188036960929537799936461532303199029211244332 \\
\hline$\infty$ & $2.40521881719(7)$
\end{tabular}

Table 36. Critical point $v_{\mathrm{c}}$ of the $q=3$ state Potts model on the three-twelve medial lattice.

\begin{tabular}{l|l}
$n$ & $v_{\mathrm{c}}$ \\
\hline 1 & 2.7176916926829055691824338364721435254533774542481 \\
2 & 2.7178413379520479881792003331093087495269320730958 \\
3 & 2.7178581401378053862927765036267716737456525642391 \\
4 & 2.7178603686953558608313898940710121620288427512401 \\
5 & 2.7178608441674816937339597619859108606883960648273 \\
6 & 2.7178609868443710417441879453116940146104031301600 \\
7 & 2.7178610401487845292293333134497014710797779301550 \\
\hline$\infty$ & $2.7178610889(3)$
\end{tabular}

Table 37. Critical point $v_{\mathrm{c}}$ of the $q=4$ state Potts model on the three-twelve medial lattice. 


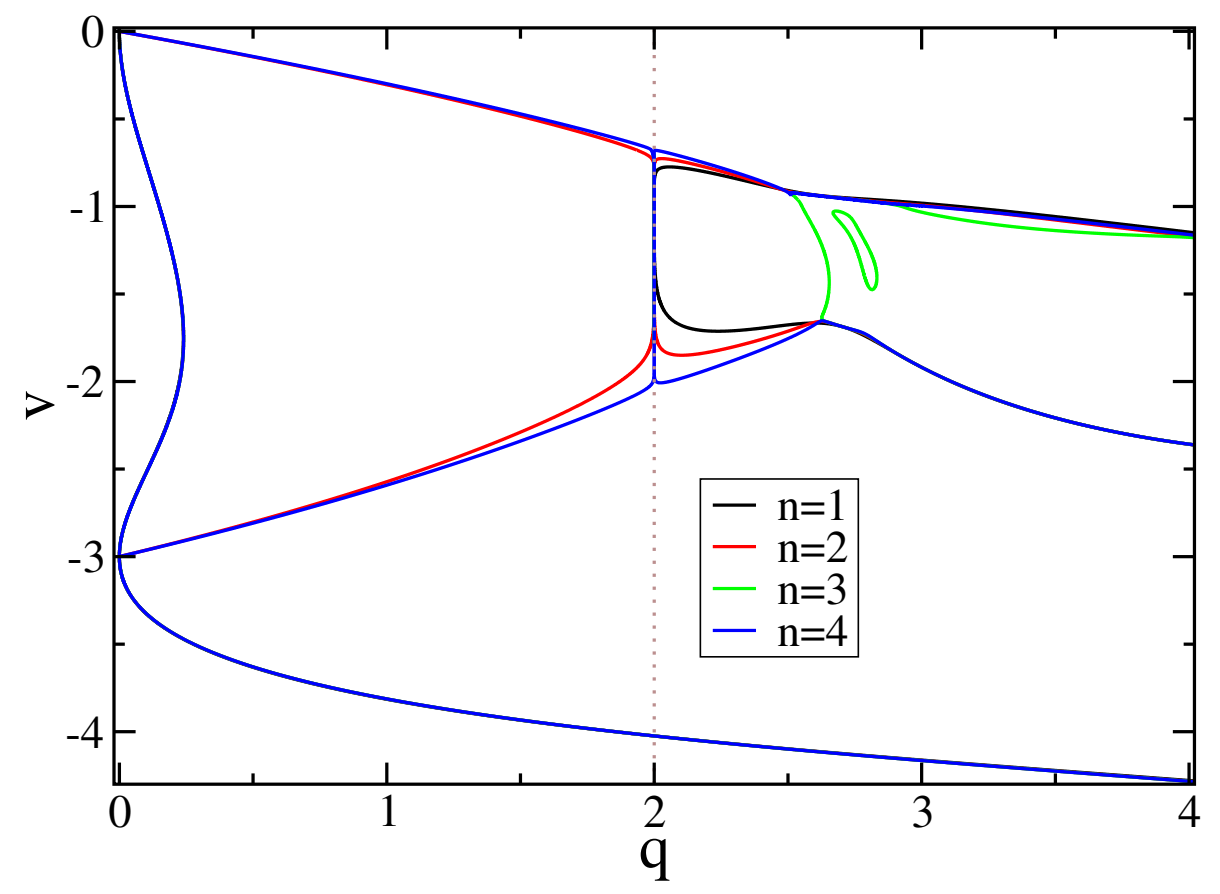

Figure 32. Roots of $P_{B}(q, v)$ for the Potts model on the three-twelve medial lattice, using $n \times n$ square bases.

The critical points $v_{\mathrm{c}}$ for the $q=3$ and $q=4$ state Potts models are given in Tables 36-37.

Despite the very large size of the bases, the phase diagram of the three-twelve medial lattice (see Figure 32) is not very complicated. The upper and lower boundaries $v_{ \pm}(q)$ of the BK phase are a couple of curves going out of the points $(q, v)=(0,0)$ and $(0,-3)$, respectively, with finite slopes. They join via an arc at $q \approx 2.6$ which is however only visible in the $n=3$ result. Accordingly the only vertical ray is at $q=B_{4}=2$. The role of the elongated bubble at $q \approx 2.7$ in the $n=3$ curve is not clear and would have to be confirmed at larger sizes. An additional, lowest lying curve goes out of $(q, v)=(0,-3)$ vertically and continues to large $q$; this curve is outside the BK phase since it does not touch the vertical ray at $q=2$.

Note that the large degree of the polynomials $P_{B}(q, v)$ makes the computation very memory demanding, so that, at variance with the general rule, we have not computed the case $n=5$ for this lattice.

\subsection{Cross medial lattice $\mathcal{M}(4,6,12)$}

Figure 33 shows a four-terminal representation of the cross medial lattice. The R-matrix is identical to that of the four-eight medial lattice (see section 6.1) for grey squares where at least one of $x$ and $y$ is even. When both $x$ and $y$ are odd the $\check{R}$-matrix is simply the identity. This corresponds formally to setting the coupling constant to infinity on two of the edges, and is represented by a coil-like symbol in the figure. We have therefore $\frac{9}{2}$ 


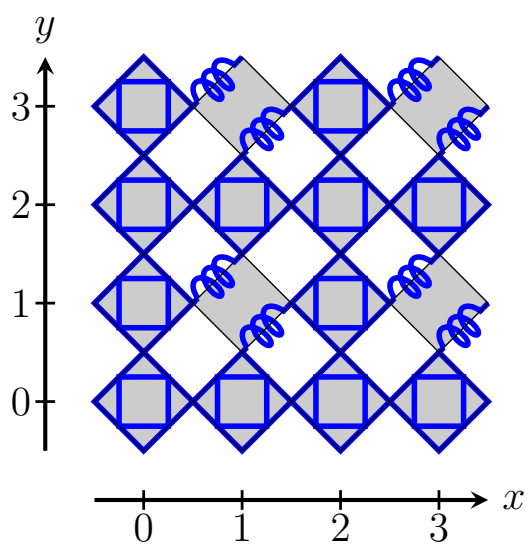

Figure 33. Four-terminal representation of the cross medial lattice. The coil-like symbols 10 indicate couplings of infinite strength $(x=\infty)$, which amount to identifying the corresponding end points.

\begin{tabular}{l|l}
$n$ & $p_{\mathrm{c}}$ \\
\hline 2 & 0.55937724024723794256962108611047506283318610938956 \\
4 & 0.55932369573589496957440303330493818296148431924351 \\
6 & 0.55931723978375762066932675944175473744208276838550 \\
\hline$\infty$ & $0.5593140(2)$ \\
Ref. [64, 65] & $0.559315(1)$
\end{tabular}

Table 38. Bond percolation threshold $p_{\mathrm{c}}$ on the cross medial lattice.

vertices and 9 edges per grey square. Note that this representation is defined only for even $n$.

The reader might want to verify the presence of hexagons and dodecagons in Figure 33, apart from the obvious squares. All of these polygons share each of their edges with a triangle, as they should, since the underlying (4,6,12)-lattice is a cubic graph.

The percolation thresholds $p_{\mathrm{c}}$ are given in Table 38 .

For the Ising model, the largest degree of the factors is $d_{\max }=16$ for both $n=2$ and $n=4$. The factor relevant for determining $v_{\mathrm{c}}$ simplifies upon setting $v=-1+\sqrt{y}$ and becomes

$$
-647-2192 y-2700 y^{2}-1952 y^{3}-594 y^{4}-80 y^{5}-28 y^{6}+y^{8} .
$$

The relevant root cannot be written simply, but reads numerically $v_{\mathrm{c}} \simeq 1.726376028 \cdots$.

The critical points for the $q=3$ and $q=4$ state Potts models are displayed in Tables 39-40.

The phase diagram of the cross medial lattice, shown in Figure 34, is rather simple. The upper and lower boundaries $v_{ \pm}(q)$ of the BK phase go out of the points $(q, v)=(0,0)$ and $(0,-3)$ with finite slope. Those curves do not continue beyond the vertical ray at 
High-precision percolation thresholds and Potts-model critical manifolds

\begin{tabular}{l|l}
$n$ & $v_{\mathrm{c}}$ \\
\hline 2 & 2.0661192719652071527256369125640867737169955530184 \\
4 & 2.0664267060174541103892930833168592835252221310632 \\
6 & 2.0664638219492513119758793783459064888440440205083 \\
\hline$\infty$ & $2.0664824(5)$
\end{tabular}

Table 39. Critical point $v_{\mathrm{c}}$ of the $q=3$ state Potts model on the cross medial lattice.

\begin{tabular}{l|l}
$n$ & $v_{\mathrm{c}}$ \\
\hline 2 & 2.3476220668229052461072103127486172282397282948400 \\
4 & 2.3482125007252188243553453531545206790126600180109 \\
6 & 2.3482844812797068752986152370611930911819486107845 \\
\hline$\infty$ & $2.3483209(7)$
\end{tabular}

Table 40. Critical point $v_{\mathrm{c}}$ of the $q=4$ state Potts model on the cross medial lattice.

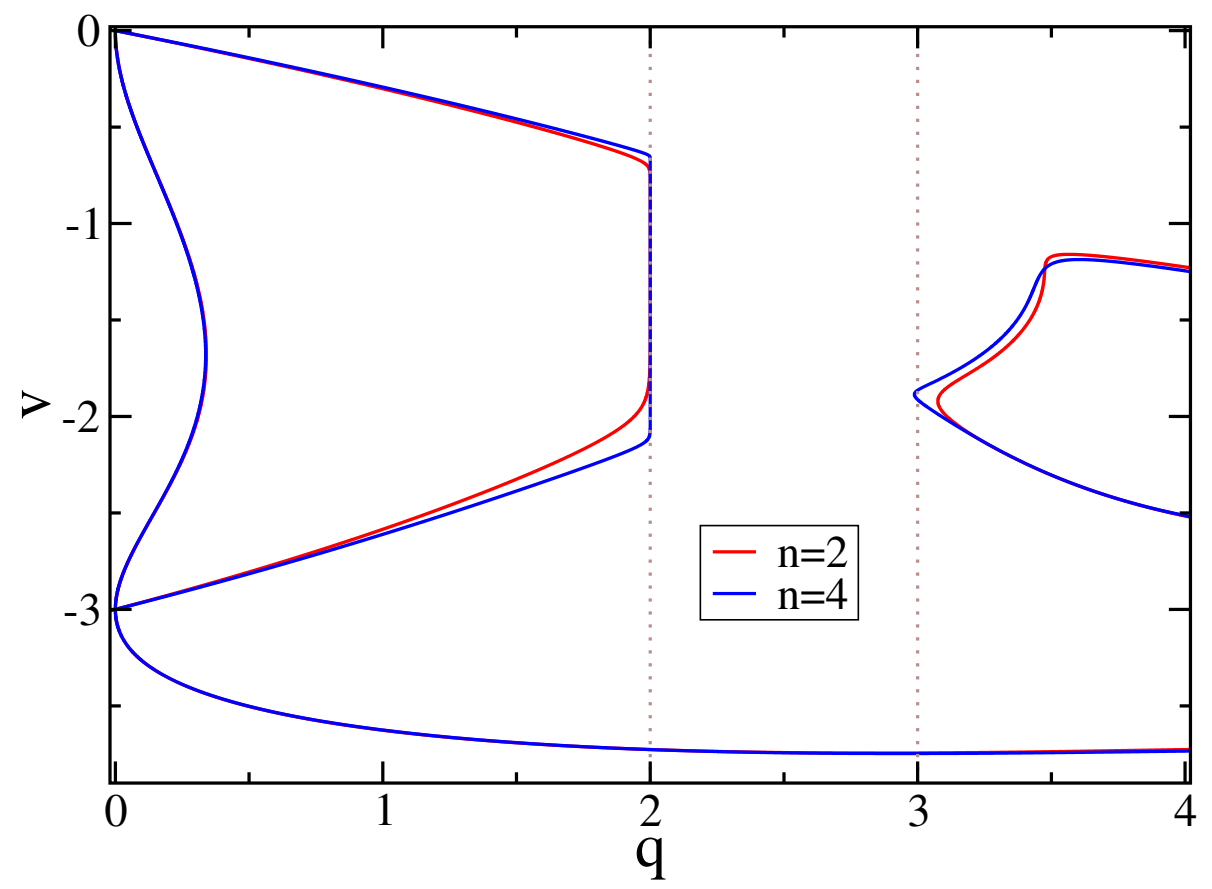

Figure 34. Roots of $P_{B}(q, v)$ for the Potts model on the cross medial lattice, using $n \times n$ square bases. 


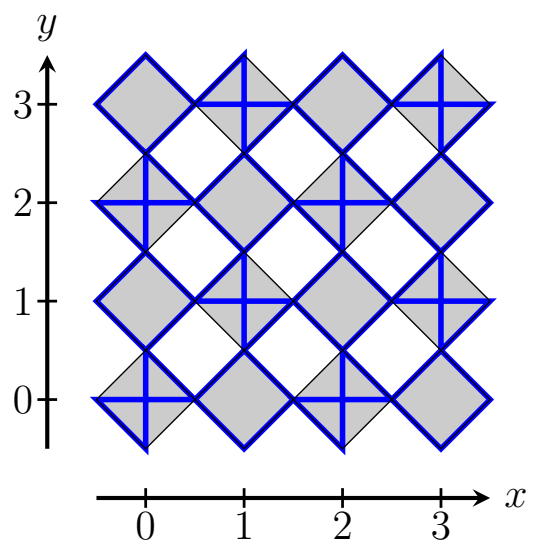

Figure 35. Four-terminal representation of the snub square medial lattice.

\begin{tabular}{l|l}
$n$ & $p_{\mathrm{c}}$ \\
\hline 2 & 0.51268177007416705104691620130181855898975396855381 \\
4 & 0.51268265882717082159880177253322350153155873133318 \\
6 & 0.51268281383012303050037916209722508801359877315417 \\
\hline$\infty$ & $0.512682929(8)$
\end{tabular}

Table 41. Bond percolation threshold $p_{\mathrm{c}}$ on the snub square medial lattice.

$q=B_{4}=2$, but from the experience with other lattices we can safely assume that this is an artefact of our choice of bases. In particular, since the $q=2$ ray has finite length, the BK phase must extend further to the right. Presumably it ends at the arc extending from $q \approx 3.0$ to $q \approx 3.4$. If so, we would expect a further vertical ray at $q=B_{6}=3$ to build up for larger bases (note that the lower end point of the arc in the $n=4$ curve is conspicuously close to $q=3$ ). Finally, there is another, lowest lying curve going out of $(q, v)=(0,-3)$ vertically which is outside the BK phase.

\subsection{Snub square medial lattice $\mathcal{M}\left(3^{2}, 4,3,4\right)$}

A four-terminal representation of the snub square medial lattice is shown in Figure 35. Its $\check{\mathrm{R}}$-matrix is a mixture of known ingredients: It is given by $\check{\mathrm{R}}_{i}$ of the square lattice, eq. (25), when $x+y$ is odd; by that of the kagome lattice, eq. (29), when $x$ and $y$ are both even; and by the alternative kagome representation discussed in section 6.1 and depicted in Figure 26 when $x$ and $y$ are both odd. This representation contains $\frac{5}{2}$ vertices and 5 edges per grey square. Once again we must require $n$ to be even.

The approximate percolation thresholds $p_{\mathrm{c}}$ obtained from the positive root of $P_{B}(p)$ are displayed in Table 41.

The polynomials $P_{B}(q, v)$ for the Ising model $(q=2)$ factorise, and the maximum degree of the factors is $d_{\min }=16$ for $n=2,4$. However, if we perform the change of 


\begin{tabular}{l|l}
$n$ & $v_{\mathrm{c}}$ \\
\hline 2 & 1.8076912391671183952391787449901823591647820014398 \\
4 & 1.8076887583089533312504288196364050004751764498759 \\
6 & 1.8076880495316679240666496789871554448835168665187 \\
\hline$\infty$ & $1.80768699(4)$
\end{tabular}

Table 42. Critical point $v_{\mathrm{c}}$ of the $q=3$ state Potts model on the snub square medial lattice.

\begin{tabular}{l|l}
$n$ & $v_{\mathrm{c}}$ \\
\hline 2 & 2.0825184394311798898958915154426893632024045546203 \\
4 & 2.0825149857011588692125250315589456916982608757655 \\
6 & 2.0825137172947986829507608673283764173966544463916 \\
\hline$\infty$ & $2.0825105(3)$
\end{tabular}

Table 43. Critical point $v_{\mathrm{c}}$ of the $q=4$ state Potts model on the snub square medial lattice.

variables $v=-1+\sqrt{y}$, the polynomial determining $y$ is only of degree 8 :

$$
1-72 y-304 y^{2}-320 y^{3}-226 y^{4}-88 y^{5}-16 y^{6}+y^{8} \text {. }
$$

The largest positive root in $y$ corresponds to the unique positive root in $v$, which is $v_{\mathrm{c}} \simeq 1.480593024 \cdots$.

Critical points of the $q=3$ and $q=4$ state Potts models are given in Tables $42-43$.

The phase diagram of the snub square medial lattice is shown in Figure 36. There are vertical rays at $q=B_{k}$ with $k=4,6,8$. The gap in the rendering of the boundaries $v_{ \pm}(q)$ of the BK phase for $2<q<3$ is partly filled out by the bubble appearing in the $n=4$ curve. The curve coming out of $(q, v)=(0,0)$ with infinite slope bends around and goes through the point $(q, v)=(-3,0)$. Just below the latter point we notice the formation of several narrow fingers which might well tend to be space filling at larger $n$, in analogy with what was observed for the frieze medial lattice (see Figure 30). Finally, in the bottom of the phase diagram there is an almost-horizontal curve that lies below the BK phase and extends out to large $q$.

\subsection{Snub hexagonal medial lattice $\mathcal{M}\left(3^{4}, 6\right)$}

Recall from section 4.9 that for the snub hexagonal lattice we have computed $P_{B}(q, v)$ using two different methods. Also for the corresponding medial lattice shall we present two distinct constructions.

The first construction proceeds in analogy with that of the snub hexagonal lattice itself. Just like the latter was obtained in Section 4.9 as a depleted version of the triangular lattice, we can construct its medial by depleting the kagome (medial of the triangular) lattice. The $\mathrm{H}$ and $\mathrm{V}$ operators corresponding to erased edges are replaced 


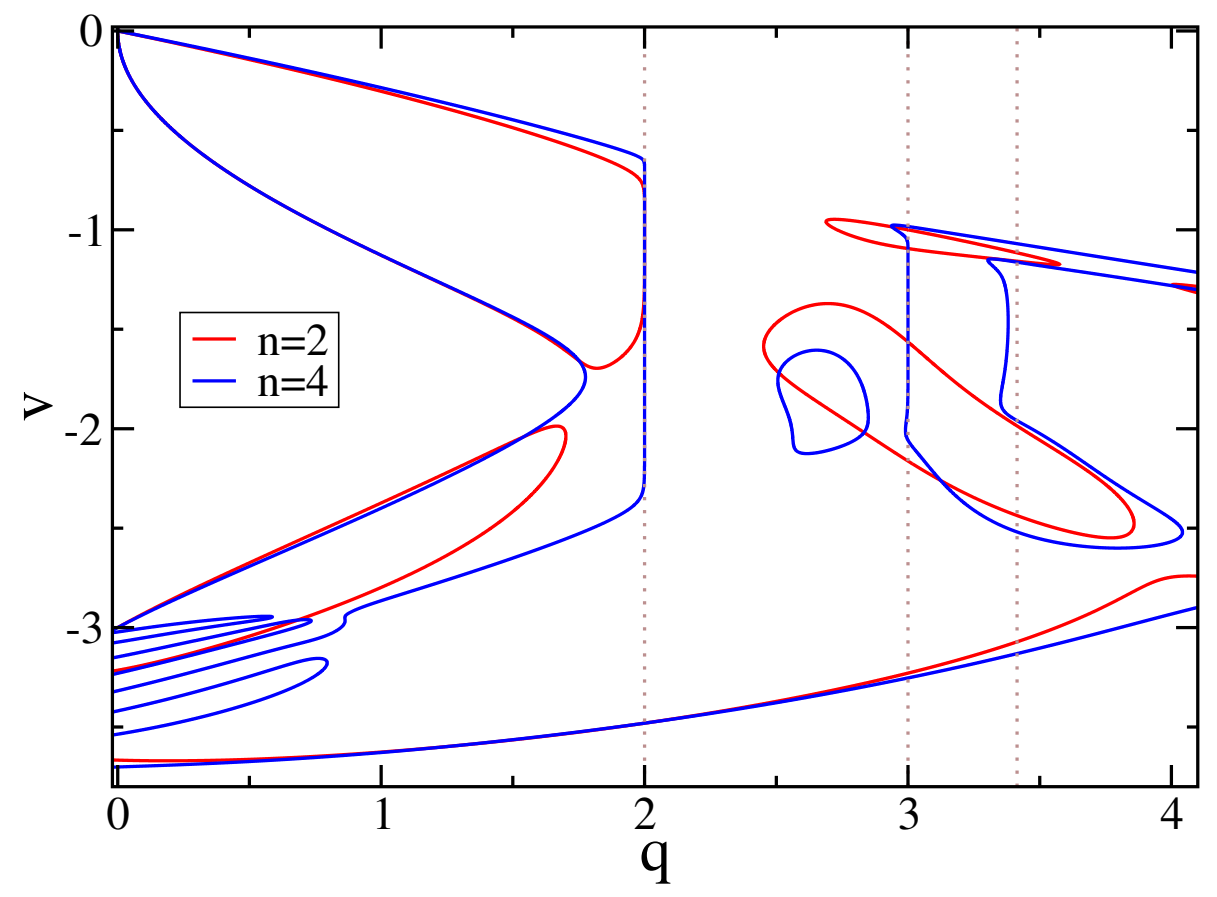

Figure 36. Roots of $P_{B}(q, v)$ for the Potts model on the snub square medial lattice, using $n \times n$ square bases.

by just one of their two terms (the identity Id or the Temperley-Lieb generator $\mathrm{E}$, as the case may be). We thus obtain $\frac{15}{7}$ vertices and $\frac{30}{7}$ edges per grey square.

In formal terms, the $\check{R}$-matrix reads:

$$
\check{\mathrm{R}}= \begin{cases}\mathrm{H}_{i+1} \mathrm{~V}_{i+2} \mathrm{~V}_{i} \mathrm{E}_{i+1} \mathrm{~V}_{i+2} \mathrm{~V}_{i} \mathrm{H}_{i+1} & \text { for } 3 y+x=0,3,6 \bmod 7 \\ \mathrm{Id}_{i+1} \mathrm{~V}_{i+2} \mathrm{~V}_{i} \mathrm{E}_{i+1} \mathrm{~V}_{i+2} \mathrm{~V}_{i} \mathrm{H}_{i+1} & \text { for } 3 y+x=1 \bmod 7 \\ \mathrm{E}_{i+1} \mathrm{~V}_{i+2} \mathrm{E}_{i} \mathrm{E}_{i+1} & \text { for } 3 y+x=2 \bmod 7 \\ \mathrm{E}_{i+1} \mathrm{E}_{i+2} \mathrm{~V}_{i} \mathrm{E}_{i+1} & \text { for } 3 y+x=4 \bmod 7 \\ \mathrm{H}_{i+1} \mathrm{~V}_{i+2} \mathrm{~V}_{i} \mathrm{E}_{i+1} \mathrm{~V}_{i+2} \mathrm{~V}_{i} \mathrm{Id}_{i+1} & \text { for } 3 y+x=5 \bmod 7\end{cases}
$$

The first line in (67) corresponds to undepleted bow tie patterns in the grey squares, i.e., it coincides with (29). Subsequent lines are obtained from the first one by depletion, i.e., replacing some of the $\mathrm{H}$ operators by the identity $\mathrm{Id}$, and some of the $\mathrm{V}$ operators by the Temperley-Lieb generator $\mathrm{E}$.

As with the snub hexagonal lattice itself (see section 4.9), the representation (49) only makes sense when $n$ is a multiple of 7 . So the only computation that we can perform in practice is to find numerically the roots in $v$ of $P_{B}(q, v)=0$ with $n=7$. We therefore turn now to an alternative construction.

This second construction is based on the four-terminal representation shown in Figure 37. The periodicity of the tiling is 3 horizontally and 6 vertically, so we can use it with rectangular bases of size $n \times 2 n$ provided that $n=0 \bmod 3$. Like the snub square medial lattice (see Figure 35) it uses both the kagome bow tie and its rotated counterpart. More precisely, for even $y$ the $\check{R}$-matrix is given by (29) when 


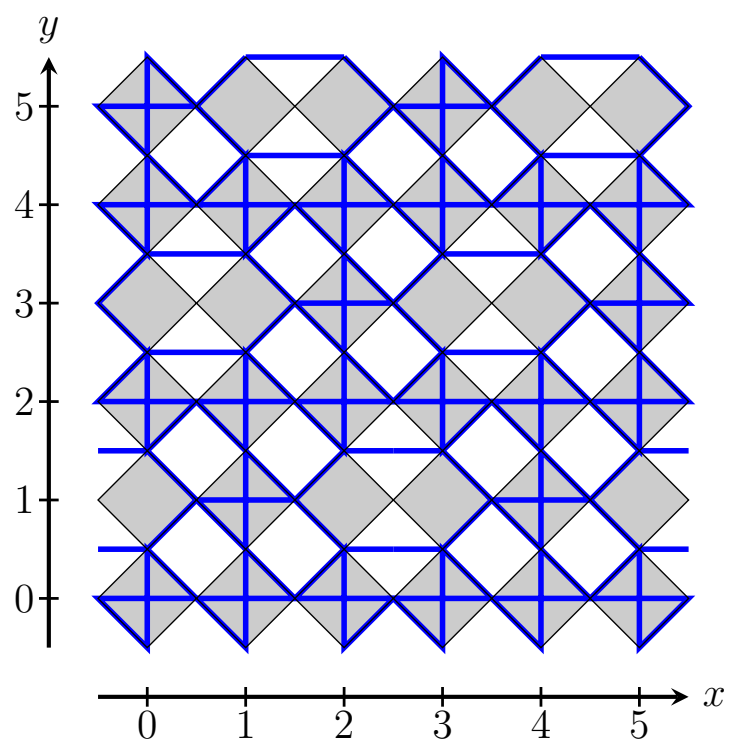

Figure 37. Four-terminal representation of the snub hexagonal medial lattice.

\begin{tabular}{l|l}
$n$ & $p_{\mathrm{c}}$ \\
\hline 3 & 0.52475737762284412234924602775712228115650254824673 \\
6 & 0.52475180898647697016696829398686817893243747112660 \\
\hline 7 & 0.52475071084639438962284356792715186350322362744063 \\
\hline$\infty$ & $0.5247495(5)$
\end{tabular}

Table 44. Bond percolation threshold $p_{\mathrm{c}}$ on the snub hexagonal medial lattice.

$x+y=0,1 \bmod 3$, and is that of the alternative kagome representation (see Figure 26) when $x+y=2 \bmod 3$. For odd $y$ we have

$$
\check{\mathrm{R}}_{i}= \begin{cases}\mathrm{E}_{i+2} \mathrm{~V}_{i} \mathrm{H}_{i+1} & \text { for } x+y=0 \bmod 3 \\ \mathrm{H}_{i+1} \mathrm{~V}_{i+2} \mathrm{E}_{i} & \text { for } x+y=1 \bmod 3 \\ \text { Eq. }(29) & \text { for } x+y=2 \bmod 3\end{cases}
$$

Moreover, one must place a horizontal diagonal on the white squares having coordinates $\left(x+\frac{1}{2}, y+\frac{1}{2}\right)$ whenever $x+2\lfloor y / 2\rfloor=2 \bmod 3$. The reader should check that each hexagon (which contains two of the horizontal diagonals) shares each of its edges with a pentagon and each of its vertices with a triangle.

This construction provides $\frac{5}{2}$ vertices and 5 edges per grey square. The packing density has therefore been improved with respect to the first construction. However, due to the parity constraint on $n$ we can now only attain $n=6$, instead of $n=7$.

In Table 44 we show the percolation thresholds $p_{\mathrm{c}}$ using both the first and the second constructions. We have separated the results using the two different constructions by a horizontal line in this and the following two tables. Extrapolating the results with $n=3,6$ leads to the estimate for the $n \rightarrow \infty$ limit shown in the last line of Table 44 . We have here supposed that the parameter $w$ in (30) is in the same range, up to a confidence 


\begin{tabular}{l|l}
$n$ & $v_{\mathrm{c}}$ \\
\hline 3 & 1.87441224209231315831992290889569424963206638843069 \\
6 & 1.87444613270433948971046627661542407584315443408254 \\
\hline 7 & 1.87445210254248307019647275740812225826172420927313 \\
\hline$\infty$ & $1.874472(5)$
\end{tabular}

Table 45. Critical point $v_{\mathrm{c}}$ of the $q=3$ state Potts model on the snub hexagonal medial lattice.

\begin{tabular}{l|l}
$n$ & $v_{\mathrm{c}}$ \\
\hline 3 & 2.15207135493272709295185607677925040508589248866351 \\
6 & 2.15213853284896945377792212011213016797276961979614 \\
\hline 7 & 2.15214995613273843394699263476921693525626146414824 \\
\hline$\infty$ & $2.15219(1)$
\end{tabular}

Table 46. Critical point $v_{\mathrm{c}}$ of the $q=4$ state Potts model on the snub hexagonal medial lattice.

interval of \pm 0.2 , as that found for the closely related snub square medial lattice (see section 6.5). The distance of the $n=7$ result to the final value is consistent with our scaling analysis.

In the case of the Ising model $(q=2)$ the polynomials $P_{B}(q, v)$ with $n=3$ factorises. The degrees of the largest factors are $1,4,6,12$ and $d_{\max }=44$. We note that the value of $d_{\max }$ is unusually large. The factor leading to a positive real root is the one of degree 4. It simplifies upon setting $v=-1+\sqrt{y}$, becoming

$$
-3-6 y+y^{2} \text {. }
$$

Our conjecture for the critical point is thus

$$
v=-1+\sqrt{3+2 \sqrt{3}} \simeq 1.542459756 \cdots
$$

and this is seen to coincide with the result (56) for the ruby lattice. By means of a 50 -digit numerical computation we have verified that the number (70) is also a root of the $n=6$ polynomial, and of the $n=7$ polynomial arising from the first construction. This provides compelling evidence that, on one hand, the factor (69) is recurrent for all of the critical polynomials, and, on the other hand, that our two different constructions lead to consistent results.

The results for the critical point $v_{\mathrm{c}}$ in the $q=3$ and $q=4$ state Potts models are shown in Tables 45-46. Also in those cases have we based the $n \rightarrow \infty$ extrapolations on the results coming from the second construction (with $n=3,6$ ), and the approach is similar to that described above for $q=1$. Again, the $n=7$ results confirm our scaling analysis.

The phase diagram of the snub hexagonal medial lattice is shown in Figure 38 . Unfortunately this is based on the single size $n=3$, so we cannot make very firm 


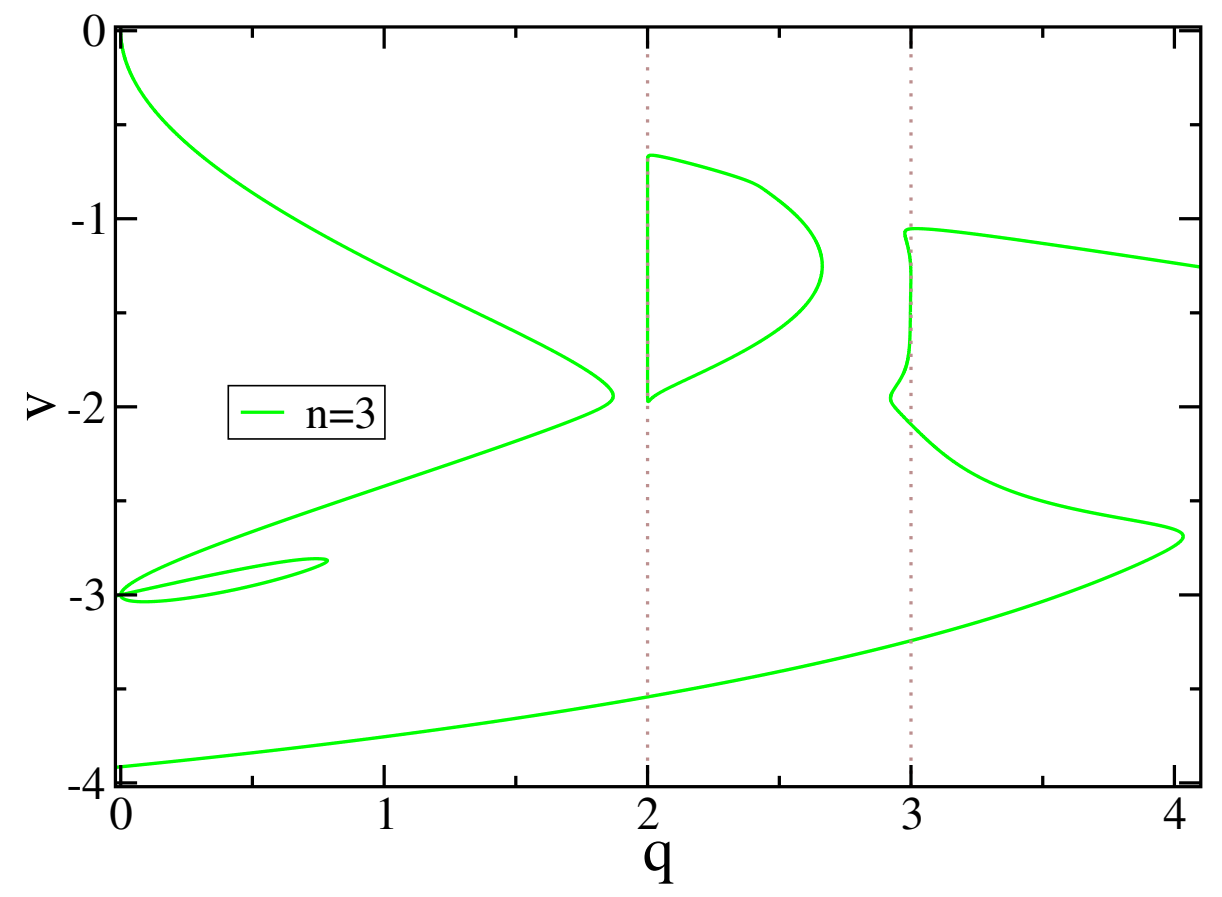

Figure 38. Roots of $P_{B}(q, v)$ for the Potts model on the snub hexagonal medial lattice, using $n \times 2 n$ rectangular bases.

statements about the convergence properties. The usual vertical rays are visible at $q=B_{k}$ with $k=4,6$. The rightmost arc of the bubble containing the $q=2$ vertical ray may well be a precursor of the $v_{ \pm}(q)$ curves on the interval $2<q<3$. On the other hand, the part of $v_{+}(q)$ with $0<q<2$ is clearly missing with this choice of basis. There is a narrow finger close to the point $(q, v)=(0,-3)$ through which the curve passes twice.

\subsection{Ruby medial lattice $\mathcal{M}(3,4,6,4)$}

The ruby medial lattice can be represented in four-terminal form as shown in Figure 39. This representation is valid only when $n$ is a multiply of 4 , and so we shall be limited to studying the case $n=4$ in the following.

As discussed in section 3.4 one can act on the two strands in a white square by the operator $\mathrm{H}_{i}$ in order to produce a horizontal diagonal. This has been done here on the white squares with coordinates $(x, y)=\left(\frac{3}{2}, \frac{1}{2}\right),\left(\frac{3}{2}, \frac{3}{2}\right),\left(\frac{7}{5}, \frac{5}{2}\right),\left(\frac{7}{2}, \frac{7}{2}\right) \bmod 4$. But for the present lattice we shall also need a slight variation of this trick, namely to act with the operator $\mathrm{E}_{i}$ instead. This corresponds to formally setting the coupling strength $x=\infty$, and renormalising by a factor of $x$, meaning that the two sites sitting across the horizontal diagonal of the white square will be effectively identified, or contracted. This operation is represented by a coil-like symbol in Figure 39, and we use it in the white squares with coordinates $(x, y)=\left(\frac{1}{2}, \frac{1}{2}\right),\left(\frac{1}{2}, \frac{3}{2}\right),\left(\frac{5}{2}, \frac{5}{2}\right),\left(\frac{5}{2}, \frac{7}{2}\right)$ mod 4 . The reader may want to verify from the figure that each triangle is surrounded by six squares (three 


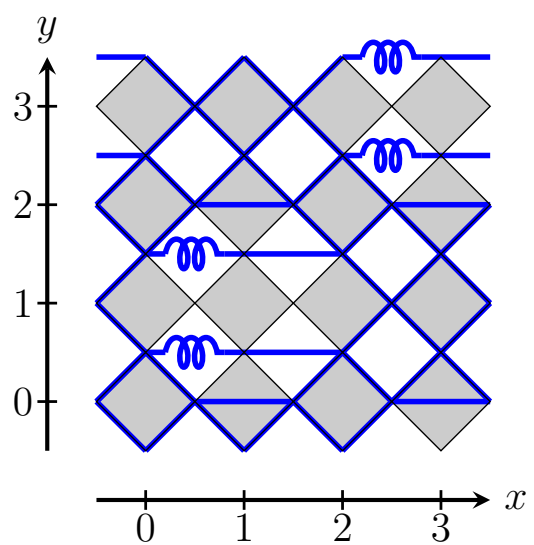

Figure 39. Four-terminal representation of the ruby medial lattice.

\begin{tabular}{l|l}
$n$ & $p_{\mathrm{c}}$ \\
\hline 4 & 0.51276405773159089981168194057612883705509718962342
\end{tabular}

Table 47. Bond percolation threshold $p_{\mathrm{c}}$ on the ruby medial lattice.

of which share an edge with the triangle, and the other three share only a vertex), and similarly that each hexagon is surrounded by twelve squares (that again alternate between edge-sharing and vertex-sharing). Each hexagon has been represented as an octagon with two $x=\infty$ edges.

Similarly, some of the edges in the grey squares have coupling strength $x=0$, i.e., they reduce to operators Id or E. Thus, each hexagon comprises two spins and one dual spin that are "free", in the sense that they do not interact with the remainder of the lattice. Since each $4 \times 4$ pattern contains two hexagons, this leads to a extraneous factor $q^{6}$ by which we must divide in order to form $P_{B}(q, v)$. Obviously the occurrences of $x=0$ or $x=\infty$ do not count as edges of the lattice that we are investigating, and therefore diminish the efficiency of the representation. In the present case we have therefore a rather modest number of $\frac{3}{2}$ vertices and 3 edges per grey square.

The R-matrix acting on the grey squares can be formally described as

$$
\check{\mathrm{R}}_{i}= \begin{cases}\mathrm{H}_{i+1} \mathrm{~V}_{i} \mathrm{~V}_{i+2} \mathrm{H}_{i+1} & \text { if }(x, y)=(0,0) \bmod 2 \\ \mathrm{H}_{i+1} \mathrm{~V}_{i} \mathrm{~V}_{i+2} \mathrm{H}_{i+1} & \text { if }(x, y)=(1,3) \text { or }(3,1) \bmod 4 \\ \mathrm{E}_{i} \mathrm{H}_{i+1} \mathrm{~V}_{i+2} \mathrm{H}_{i+1} & \text { if }(x, y)=(1,0) \text { or }(3,2) \bmod 4 \\ \mathrm{H}_{i+1} \mathrm{~V}_{i} \mathrm{H}_{i+1} \mathrm{E}_{i+2} & \text { if }(x, y)=(3,0) \text { or }(1,2) \bmod 4 \\ \mathrm{~V}_{i} \mathrm{E}_{i+2} \mathrm{H}_{i+1} & \text { if }(x, y)=(0,1) \text { or }(2,3) \bmod 4 \\ \mathrm{H}_{i+1} \mathrm{E}_{i} \mathrm{~V}_{i+2} & \text { if }(x, y)=(2,1) \text { or }(0,3) \bmod 4 \\ \mathrm{E}_{i} \mathrm{E}_{i+2} & \text { if }(x, y)=(1,1) \text { or }(3,3) \bmod 4\end{cases}
$$

The $n=4$ result for the percolation threshold $p_{\mathrm{c}}$ is shown in Table 47 , and those for the critical points $v_{\mathrm{c}}$ of the $q=3$ and $q=4$ state Potts models are reported in Tables 48-49. From these single data points, which moreover do not correspond to a 


\begin{tabular}{l|l}
$n$ & $v_{\mathrm{c}}$ \\
\hline 4 & 1.80131354317808391632479082679158473077866941417858
\end{tabular}

Table 48. Critical point $v_{\mathrm{c}}$ of the $q=3$ state Potts model on the ruby medial lattice.

\begin{tabular}{l|l}
$n$ & $v_{\mathrm{c}}$ \\
\hline 4 & 2.07313974259180806296373118024496389902474086148828
\end{tabular}

Table 49. Critical point $v_{\mathrm{c}}$ of the $q=4$ state Potts model on the ruby medial lattice.

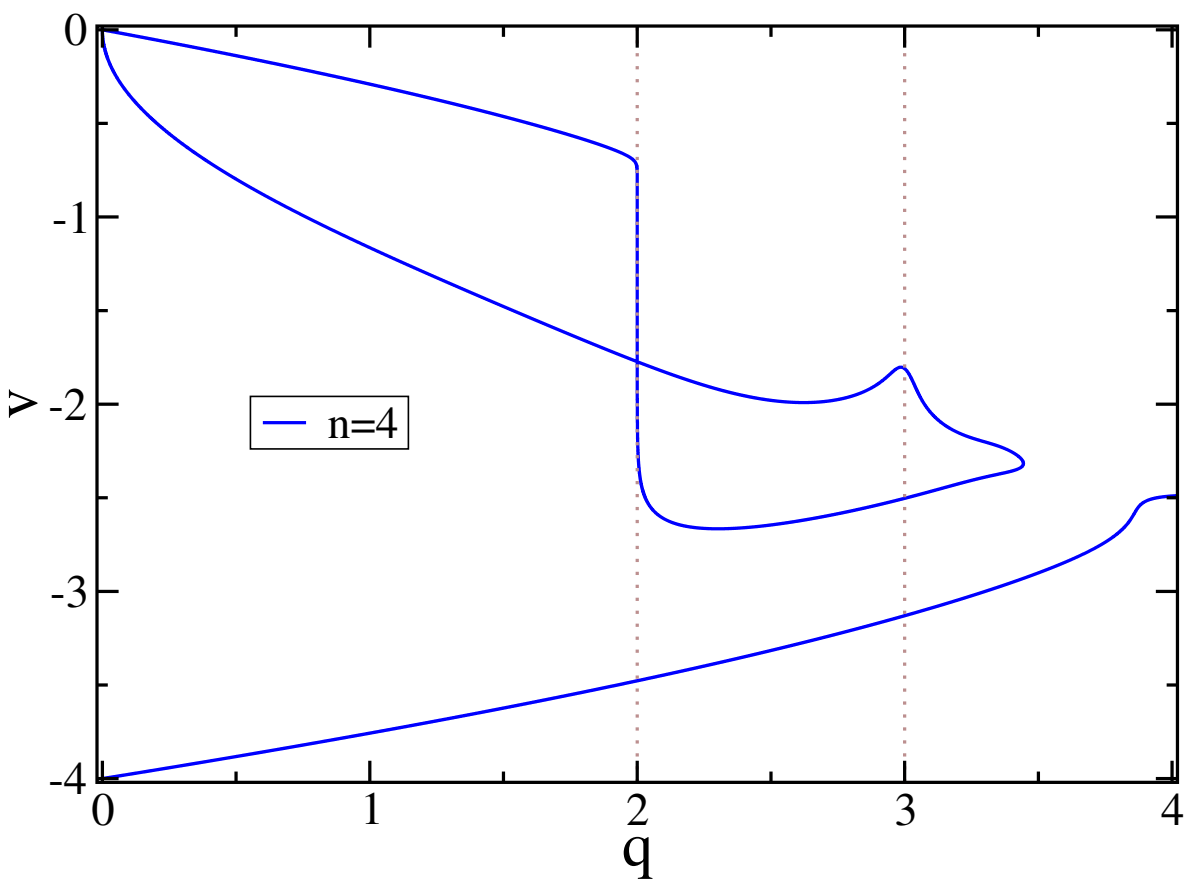

Figure 40. Roots of $P_{B}(q, v)$ for the Potts model on the ruby medial lattice, using $n \times n$ square bases.

very large size of the basis compared to the other lattices treated in this work, it does not seem reasonable to provide a final result with error bars for the $n \rightarrow \infty$ limit.

For the Ising model $(q=2)$ the factorisation of $P_{B}(q, v)$ with $n=4$ contains factors of maximum degree $d_{\max }=20$. The factor determining $v_{\mathrm{c}}$ is of degree 16 , and by setting $y=-1+\sqrt{y}$ it becomes simpler:

$$
-3-6 y-66 y^{2}-174 y^{3}-194 y^{4}-58 y^{5}-10 y^{6}-2 y^{7}+y^{8} .
$$

The largest real root in $y$ determines the critical coupling as $v_{\mathrm{c}} \simeq 1.477488025 \cdots$. Based on our experience from the Archimedean lattices we can assume this value to be the exact result.

The phase diagram in shown in Figure 40. There is a clear vertical ray at the Beraha number (21) with $k=4$, and another incipient ray with $k=6$. Note also that the $n=4$ curve passes through the point $(q, v)=(0,-4)$ exactly. The curve emanating 
from that point is presumably a good approximation to the lower boundary of the BK phase. The value of $q_{\mathrm{c}}$ is however difficult to estimate from just one curve.

\subsection{Factorisable cases}

In analogy with Section 4.11 we now discuss the cases of exact factorisation for the seven medial lattices which are not themselves Archimedean (i.e., those discussed in section 6). Statements including the words "all" or "none" thus refer to those seven lattices only.

Note that for none of the medial lattices does $P_{B}(q, v)$ factorise for generic values of $q$ and $v$. This presumably means that the Potts model is not solvable on these lattices along any curve. Conversely, factorisation does occur for isolated (integer) values of $q$ and $v$, as we now discuss.

For $q=0, P_{B}(q, v)$ factorises for all the lattices, producing a root $v=0$. The resulting free fermion theories describe spanning trees [62,61]. Moreover, the four-eight medial, frieze medial, three-twelve medial, cross medial, snub square medial and snub hexagonal medial lattices have a root $(q, v)=(0,-3)$. And the ruby medial lattice has a root $(q, v)=(0,-4)$. These are models of spanning forests [61] on the corresponding dual lattices, with weight $v$ per component tree.

Unlike the case of Archimedean lattices, we have found no medial lattices with a size-independent (finite) slope with which the curves pass through the origin $(q, v)=$ $(0,0)$.

There are a couple of unusual cases for $q=0$. The four-eight medial and cross medial lattices both shed the small factor $8+3 v+v^{2}$ with roots $v=(-3 \pm i \sqrt{23}) / 2$. Similarly, the three-twelve medial lattice sheds the factor $6+3 v+v^{2}$ with roots $(-3 \pm i \sqrt{15}) / 2$. All of these cases presumably provide loci of exact solvability.

In the Ising case $(q=2)$ there is a root in $v=-1$ (chromatic polynomial) for all the medial lattices. Unlike the case of Archimedean lattices, there are no medial lattices with a root in $v=-2$.

For $q=3$ there is a root in $v=-1$ (chromatic polynomial) for the four-eight medial lattice provided that $n$ is odd (we have checked this for $n=1,3$ ), while for $n$ even (i.e., for $n=2,4)$ the curves in Figure 27 do not even come close to the point $(q, v)=(3,-1)$. However, we have seen in this study that parity effects in $n$ are extremely common, so we feel confident in conjecturing that the three-colouring problem on the four-eight medial lattice is exactly solvable. $\dagger$

For $q=3$ the frieze medial and snub hexagonal medial lattices both shed the small factor $3+3 v+v^{2}$ whose roots, $v=(-3 \pm i \sqrt{3}) / 2$, are presumably loci of exact solvability.

Finally, for $q=4$ there is a root at $v=-2$ for the frieze medial lattice.

$\dagger$ On the other hand, $v=-1$ factorises for the snub square medial lattice with $n=2$, but not with $n=4$. In this case the factorisation appears to be a fortuity for $n=2$ rather than a systematic phenomenon. 


\section{Site percolation}

The site percolation problem can be seen as the $q \rightarrow 1$ limit of a Potts model only when the latter is generalised to include multi-spin interactions [67]. It follows that site percolation is not dual to a site percolation on the dual lattice. Therefore one might in principle want to study site percolation on all the lattices on which we have treated the Potts model above, as well as on their corresponding medial lattices. This should be possible using the techniques exposed this far, combined with a few extra tricks that we expose below. For practical reasons we shall however limit the study to a subclass of Archimedean lattices and their duals, namely those having only cubic and quartic vertices, discarding also those cases [21] for which the site percolation problem is exactly solvable. This amounts to treating the seven lattices listed in Table 50.

We have already recalled in Section 2 that bond percolation on a cubic lattice $G$ is equivalent to site percolation on the corresponding medial lattice $\mathcal{M}(G)[29,30]$. By duality this extends to cases where $G$ is a triangulation. It follows in particular from Section 4.1 that site percolation is exactly solvable on the kagome lattice, with $p_{\mathrm{c}}=1-2 \sin (\pi / 18)$, cf. Eq. (24).

By the same token, site percolation on the four-eight medial, the three-twelve medial and the cross medial lattices are equivalent to bond percolation on the corresponding original (i.e., non-medial) lattices, discussed in Sections 4.4, 4.6 and 4.7 respectively. The site percolation thresholds for these three lattices can therefore be read from Tables 7 , 13 and 16.

Apart from the kagome lattice, there are a few more lattices where site percolation is exactly solvable by relatively elementary tricks. For instance, the problem on the threetwelve lattice follows from that on the kagome lattice upon replacing $p$ by $p^{2}$. And site percolation on the triangular lattice is dealt with by noticing that the percolation hulls (that live on the dual lattice) describe the well-known [44] $\mathrm{O}(n)$ loop model on the hexagonal lattice with $n=1$. Its critical point in the dense phase has monomer fugacity $K=1$. It follows that $p_{\mathrm{c}}=1 / 2$.

Site percolation on these three exactly solvable lattices (i.e., kagome, three-twelve and triangular) was discussed in [21] from the point of view of graph polynomials $P_{B}(p)$. It was found that indeed the smallest possible bases provide the exact threshold $p_{\mathrm{c}}$ and that larger bases lead to a factorisation of the exact result.

In the remainder of this section we discuss how to compute $P_{B}(p)$ for site percolation on other lattices by using a four-terminal representation and the periodic TL transfer matrix approach. This treatment of the site percolation problem has some advantages over bond percolation, but also some inconveniences. These aspects are most vividly illustrated in the case of the square lattice. Recall that in Section 6.1 we have introduced a method in which a generic Temperley-Lieb operator acts within a grey square (see Figure 4) by giving specific weights to the fourteen possible planar pairings of the strands $i, j, k, l:=i, i+1, i+2, i+3$ and $i^{\prime}, j^{\prime}, k^{\prime}, l^{\prime}$. Consider now lodging one site of the percolation problem inside each grey square of the four-terminal representation (see 
Figure 3). When that site is occupied it must connect the grey square onto the four surrounding grey squares that it touches at its corners. Treating the loops as hulls of the percolation clusters, this is accomplished by choosing the pairing $\left(i i^{\prime}\right)(j k)\left(l l^{\prime}\right)\left(j^{\prime} k^{\prime}\right)$. On the other hand, when the site is empty it must disconnect the grey square from its surroundings. This is done by taking the pairing $(i j)(k l)\left(i^{\prime} j^{\prime}\right)\left(k^{\prime} l^{\prime}\right)$. The Boltzmann weights corresponding to an occupied (resp. an empty) site is taken as $p$ (resp. $1-p$ ), or equivalently as $v$ (resp. 1 ), where we have set $v=p /(1-p)$ as usual.

The advantage of this approach is that the TL operator is, in fact, not generic at all: it only gives a non-zero weight to two out of the fourteen possible pairings. Acting repeatedly on all grey squares of the basis therefore only produces a relatively small subset of all possible elements of the (periodic) TL algebra. We can exploit this by abandoning the approach of storing all Boltzmann weights in tables (using in particular the bijection of section 3.5.4 between connectivity states and integers), since many of those weights would be zero anyway. Instead, we simply insert the states that are produced by the transfer matrix (with non-zero weight) in a hash table. Still for the square lattice, this enables us to treat $n \times n$ bases as large as $n=11$.

The main inconvenience is that each grey square can accommodate only a relatively small number of sites (e.g., just one for the square lattice). Moreover, we have found no meaningful way of using the white squares. We shall also see below that the rate of convergence of the estimates for $p_{\mathrm{c}}$ is noticeably slower for site percolation than for bond percolation. Still, our method leads to final results that are generally at least as precise as those of the best available Monte Carlo simulations.

Throughout this section we have computed the exact percolation polynomials $P_{B}(p)$ for all sizes $n$ discussed. These polynomials are available in electronic form as supplementary material to this article. $\dagger$ In the following subsections we tabulate as usual the positive root $p_{\mathrm{c}} \in(0,1)$ of $P_{B}(p)$ to 50 decimal digits, but it should be kept in mind that in all cases these numbers are in fact known to arbitrary precision. In contrast with the bond percolation thresholds and Potts model critical points tabulated in the preceding sections, we have here not pushed the computations to larger sizes by seeking a purely numerical evaluation of the root $p_{\mathrm{c}}$.

The degree of the critical polynomials $P_{B}(p)$ for site percolation on the lattices studied below is shown in Table 50. We also provide the largest size $n_{\max }$ for which we have been able to compute the polynomial $P_{B}(p)$ for each lattice, as well as the corresponding number of vertices $|V|_{\max }$ in $B$ (which is also the degree of $P_{B}(p)$ ). The table also gives the dimension $d_{\max }$ of the largest transfer matrix used in the computation, i.e., the maximum number of states required at any intermediate stage. These dimensions should be compared with the number $\operatorname{dim}(n, 2)$ in the generic case (see Table 2), from which the advantage of the hashing approach can be judged.

$\dagger$ This supplementary material takes the form of a text file PB.m that can be processed by MAтнемAтісA or similar programs for symbolic algebra manipulations. 


\begin{tabular}{l|ll|rrr} 
Lattice & Vertices & Parity of $n$ & $n_{\max }$ & $|V|_{\max }$ & $d_{\max }$ \\
\hline Hexagonal & 2 & Any & 8 & 128 & 311467520 \\
Square & 1 & Any & 11 & 121 & 1770114092 \\
Four-eight & 4 & Any & 7 & 196 & 199753311 \\
Cross & 3 & Any & 8 & 192 & 605394138 \\
Ruby & $\frac{3}{4}$ & 0 mod 4 & 16 & 192 & 843378845 \\
Cairo pentagonal & $\frac{3}{2}$ & Even & 8 & 96 & 32215001 \\
Frieze dual & $\frac{3}{2}$ & Any & 9 & 243 & 339644725 \\
\hline
\end{tabular}

Table 50. Number of vertices per grey square (cf. Figure 2) for site percolation on various lattices, using square bases of size $n \times n$ grey squares ( ${ }^{\dagger}$ or rectangular bases of size $n \times 2 n)$. In addition we state any parity constraint on $n$. The right part of the table shows the largest size $n_{\max }$ for which we have been able to compute the polynomial $P_{B}(p)$, the corresponding number of vertices $|V|_{\max }$ in $B$, and the dimension $d_{\max }$ of the transfer matrix.

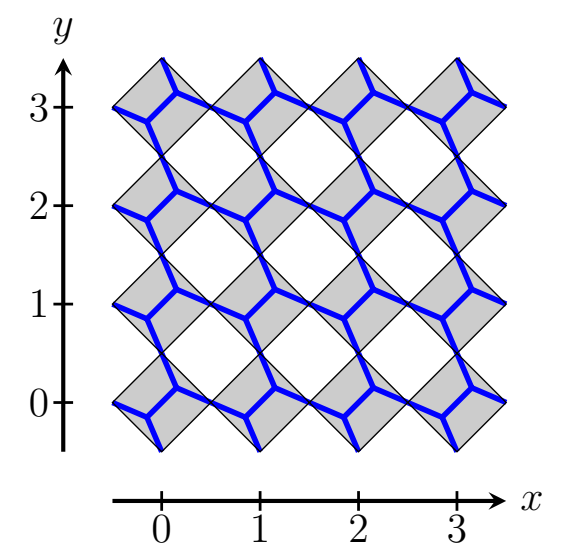

Figure 41. Four-terminal representation for site percolation on the hexagonal lattice.

\subsection{Hexagonal lattice}

Figure 41 shows a four-terminal representation for site percolation on the hexagonal lattice. There are 2 vertices per grey square.

It is useful at this stage to point out the key differences with bond percolation. In bond percolation, the "conducting units" are the edges. Since edges meet at vertices, each of the four terminals of the grey squares must be situated at the position of a vertex. On the other hand, in site percolation the "conducting units" are the vertices. It is convenient to represent an occupied site instead as a colouring of its adjacent half-edges, so that site percolation clusters become connected components (clusters) of coloured half-edges. It follows from this picture that each of the four terminals of the grey squares must be situated at the mid point of an edge.

The loop strands of Figure 4 must turn around the clusters of coloured half-edges. It follows that each choice of occupancy of sites within a grey square induces a planar 


\begin{tabular}{l|l}
$n$ & $p_{\mathrm{c}}$ \\
\hline 1 & 0.7071067811865475244008443621048490392848359376885 \\
2 & 0.6971069014219768583833477251437189263456173513781 \\
3 & 0.6971928819498649590842656967290585989087604117228 \\
4 & 0.6970613429377088940378961155082870171465188648927 \\
5 & 0.6970449503839377097642274158922485690092452929594 \\
6 & 0.6970416734307503900742086237111348168897232567095 \\
7 & 0.6970407718280774909951027270176125447620620155082 \\
8 & 0.6970404617236920725796530320181513631207407839926 \\
\hline$\infty$ & $0.697040230(5)$ \\
Ref. $[25]$ & $0.6970402(1)$
\end{tabular}

Table 51. Site percolation threshold $p_{\mathrm{c}}$ on the hexagonal lattice.

pairings of the strands $i, j, k, l:=i, i+1, i+2, i+3$ and $i^{\prime}, j^{\prime}, k^{\prime}, l^{\prime}$. In the case of Figure 41 the $\check{R}$-matrix becomes

$$
\begin{aligned}
\check{\mathrm{R}}_{i} & =(i j)(k l)\left(i^{\prime} j^{\prime}\right)\left(k^{\prime} l^{\prime}\right)+v(i l)(j k)\left(i^{\prime} j^{\prime}\right)\left(k^{\prime} l^{\prime}\right) \\
& +v(i j)(k l)\left(i^{\prime} l^{\prime}\right)\left(j^{\prime} k^{\prime}\right)+v^{2}\left(i i^{\prime}\right)(j k)\left(j^{\prime} k^{\prime}\right)\left(l l^{\prime}\right),
\end{aligned}
$$

where the first term corresponds to both sites within the grey square being empty, the second and third term correspond to one site being occupied and the other empty, and the fourth term comes from the case where both sites are occupied. This can be expressed more elegantly in terms of TL generators:

$$
\check{\mathrm{R}}_{i}=\mathrm{E}_{i+2} \mathrm{E}_{i}+v\left(\mathrm{E}_{i+2} \mathrm{E}_{i} \mathrm{E}_{i+1}+\mathrm{E}_{i+1} \mathrm{E}_{i+2} \mathrm{E}_{i}\right)+v^{2} \mathrm{E}_{i+1} .
$$

The fact that (73) contains only four terms out of fourteen possible means that the computation of $P_{B}(p)$ can be accomplished for square bases of size up to $n=8$. The corresponding thresholds $p_{\mathrm{c}}$ are shown in Table 51.

The exponent appearing in $(30)$ here comes out as $w \approx 6.35$, which is the same value as found for bond percolation on the kagome and three-twelve lattices. This seems reasonable, since all those lattices have the same three-fold rotational symmetry. $\dagger$ The high value of $w$ again entails a high precision of the final value, more than two orders of magnitude better than the best numerical result [25].

Note that graph polynomials for this lattice were previously studied in [21] for hexagonal bases with up to 54 sites (compared to the square bases with up to 128 sites used here).

$\dagger$ In particular $w$ does not seem to depend on the nature of the percolation problem (bond or site), provided that the lattice has the same symmetry. Obviously, this remark does not hold true when comparing problems that are exactly solvable with those which are not. 


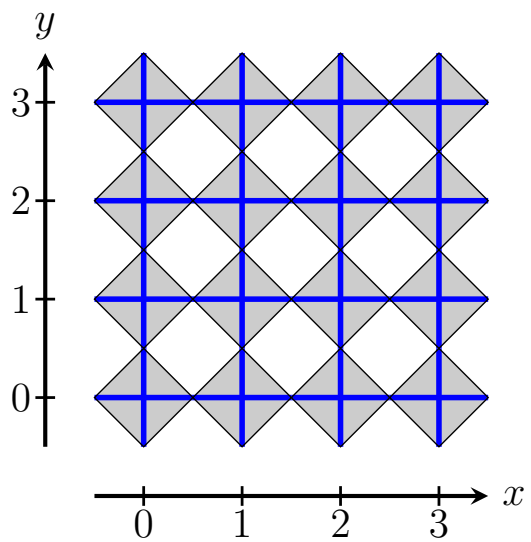

Figure 42. Four-terminal representation for site percolation on the square lattice.

\subsection{Square lattice}

Site percolation on the square lattice was already discussed as an example in the introduction to section 7. The four-terminal representation is shown in Figure 42. It has 1 vertex per grey square.

The $\check{R}$-matrix reads

$$
\check{\mathrm{R}}_{i}=(i j)(k l)\left(i^{\prime} j^{\prime}\right)\left(k^{\prime} l^{\prime}\right)+v\left(i i^{\prime}\right)(j k)\left(l l^{\prime}\right)\left(j^{\prime} k^{\prime}\right),
$$

as discussed previously. In terms of TL generators this reads

$$
\check{\mathrm{R}}_{i}=\mathrm{E}_{i+2} \mathrm{E}_{i}+v \mathrm{E}_{i+1} .
$$

Since there are only two terms out of fourteen possible, we have been able to obtain $P_{B}(p)$ for bases of size up to $n=11$. The corresponding thresholds $p_{\mathrm{c}}$ are given in Table 52 .

Since we have here eleven data points - the highest number for any of the problems treated in this paper - we have taken particular care to get the best possible extrapolation out of them. Applying the usual procedure we first found $w \approx 4.07$ from a non-linear fit (30) to the last three data points. However, it was easily detected that this choice of $w$ led to some unnecessary spread on the BS approximants. In fact, fitting successively $w$ from the last three data points among the first 9,10 or all 11 points, we found $w \approx 4.139, w \approx 4.098$ and $w \approx 4.074$, indicating that the true $w$ might be slightly lower. Repeating then the BS procedure while moving down $w$ in steps of 0.01 we have checked that the choice $w=4.03 \pm 0.01$ minimises the spread of the approximants, and hence the final value given in Table 52 is based on this choice. This is consistent with (and slightly more accurate than) the best numerical result [25].

\subsection{Four-eight lattice}

The four-terminal representation for site percolation on the four-eight lattice is shown in Figure 43. It can accommodate 4 vertices per grey square. 
High-precision percolation thresholds and Potts-model critical manifolds

\begin{tabular}{l|l}
$n$ & $p_{\mathrm{c}}$ \\
\hline 1 & 0.50000000000000000000000000000000000000000000000000 \\
2 & 0.54119610014619698439972320536638942006107206337802 \\
3 & 0.58651145511267563565455897660690173482430062489384 \\
4 & 0.59067211233102829689590201143951286962111713272216 \\
5 & 0.59198825651833384461096868021192887904787477719722 \\
6 & 0.59239507081770423769385580764250543411218819923508 \\
7 & 0.59256103742766484893896496885851283129444654611347 \\
8 & 0.59263900074535204810167646273223073775874995672901 \\
9 & 0.59267976548917331887514046884728174207445322469521 \\
10 & 0.59270280369294408906688315667089943939640050817027 \\
11 & 0.59271663223297437516334096268406621556972040226157 \\
\hline$\infty$ & $0.59274601(2)$ \\
Ref. [25] & $0.59274605(3)$
\end{tabular}

Table 52. Site percolation threshold $p_{\mathrm{c}}$ on the square lattice.

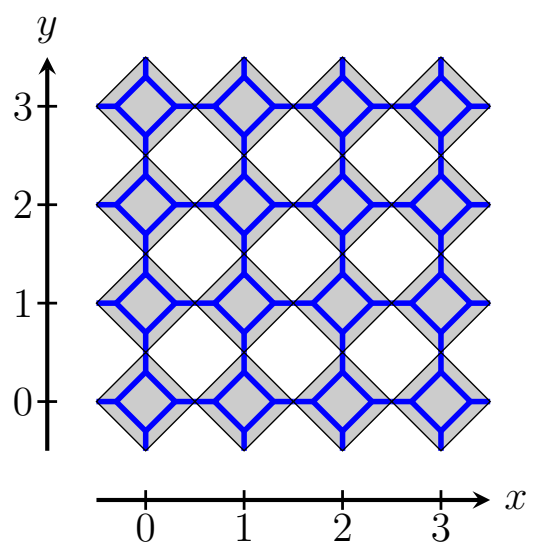

Figure 43. Four-terminal representation for site percolation on the four-eight lattice.

The $\check{R}$-matrix is now

$$
\begin{gathered}
\check{\mathrm{R}}=\left(1+4 v+2 v^{2}\right)(i j)(k l)\left(i^{\prime} j^{\prime}\right)\left(k^{\prime} l^{\prime}\right)+v^{4}\left(i i^{\prime}\right)(j k)\left(j^{\prime} k^{\prime}\right)\left(l l^{\prime}\right) \\
+v^{2}\left[\left(i i^{\prime}\right)\left(j j^{\prime}\right)(k l)\left(k^{\prime} l^{\prime}\right)+(i j)\left(i^{\prime} j^{\prime}\right)\left(k k^{\prime}\right)\left(l l^{\prime}\right)+\right. \\
\left.(i l)(j k)\left(i^{\prime} j^{\prime}\right)\left(k^{\prime} l^{\prime}\right)+(i j)(k l)\left(i^{\prime} l^{\prime}\right)\left(j^{\prime} k^{\prime}\right)\right] \\
+v^{3}\left[\left(i i^{\prime}\right)\left(j^{\prime} k^{\prime}\right)\left(j l^{\prime}\right)(k l)+\left(i i^{\prime}\right)(j k)\left(j^{\prime} l\right)\left(k^{\prime} l^{\prime}\right)+\right. \\
\left.\left(i k^{\prime}\right)(j k)\left(i^{\prime} j^{\prime}\right)\left(l l^{\prime}\right)+\left(i^{\prime} k\right)\left(j^{\prime} k^{\prime}\right)(i j)\left(l l^{\prime}\right)\right] .
\end{gathered}
$$

With ten terms out of fourteen possible, the advantage of the hashing scheme over the complete tabulation of states is less pronounced than for the lattices treated previously. Accordingly we can treat square bases of size up to $n=7$. The results for the threshold $p_{\mathrm{c}}$ are given in Table 53. 


\begin{tabular}{l|l}
$n$ & $p_{\mathrm{c}}$ \\
\hline 1 & 0.7336147478371355308558999643084317148034022526034 \\
2 & 0.7312492379002034814136736960461227248789777597490 \\
3 & 0.7301157282458717016250281495894718983091568806878 \\
4 & 0.7298412248145118289566094607464823352336030277592 \\
5 & 0.7297673439318198743116752465745127856997428287266 \\
6 & 0.7297429799645414198759930896484250713568213365103 \\
7 & 0.7297332402222111151444172392979287587234589502354 \\
\hline$\infty$ & $0.7297232(5)$ \\
Ref. [68] & $0.729724(3)$
\end{tabular}

Table 53. Site percolation threshold $p_{\mathrm{c}}$ on the four-eight lattice.

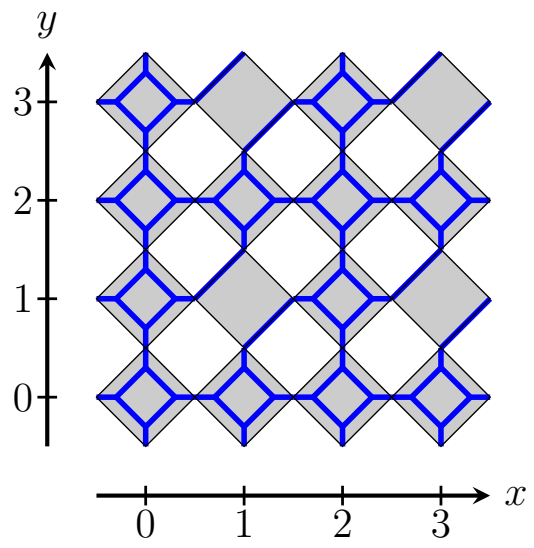

Figure 44. Four-terminal representation for site percolation on the cross lattice.

\subsection{Cross lattice}

We give a four-terminal representation for site percolation on the cross lattice in Figure 44. It requires $n$ to be even and hosts 3 vertices per grey square. The $\check{R}$ matrix is the same as for the four-eight lattice, except when $x$ and $y$ are both odd in which case it is replaced by the identity operator. One may check the presence of faces of degree 6 and 12 .

Table 54 provides the results for the site percolation threshold $p_{\mathrm{c}}$.

\subsection{Ruby lattice}

The four-terminal representation for site percolation on the ruby lattice requires some new tricks. It is shown in Figure 45, where we have supposed that $n=0 \bmod 4$. As usual the coil-like symbol indicates an edge of infinite strength, meaning that the two vertices at its end points have been identified. The states of the two grey squares linked by a coil are now correlated: if the site in one of the squares is empty (resp. occupied) the 


\begin{tabular}{l|l}
$n$ & $p_{\mathrm{c}}$ \\
\hline 2 & 0.7486176795231741957833806597419001398221099848029 \\
4 & 0.7478954923957336800827109221019572017788873033627 \\
6 & 0.7478142908979452473592066469359302051298398370653 \\
8 & 0.7478045322937317100218711372257136728840658082387 \\
\hline$\infty$ & $0.7478008(2)$ \\
Ref. [68] & $0.747806(4)$
\end{tabular}

Table 54. Site percolation threshold $p_{\mathrm{c}}$ on the cross lattice.

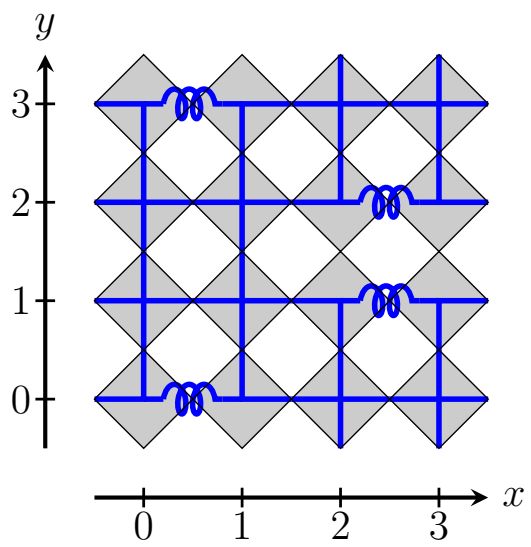

Figure 45. Four-terminal representation for site percolation on the ruby lattice.

same is true in the adjacent square, since the two sites have been identified. Identifying such conglomerates of two grey squares by the coordinates $(x, y)$ of the leftmost one, we have the following $\check{\mathrm{R}}$-matrix:

$$
\check{\mathrm{R}}_{i}= \begin{cases}\mathrm{E}_{i+4} \mathrm{E}_{i+2} \mathrm{E}_{i}+v \mathrm{E}_{i+3} \mathrm{E}_{i+4} \mathrm{E}_{i+1} \mathrm{E}_{i+2} & \text { if } x+y=0 \bmod 4 \\ \mathrm{E}_{i+4} \mathrm{E}_{i+2} \mathrm{E}_{i}+v \mathrm{E}_{i+2} \mathrm{E}_{i+3} \mathrm{E}_{i} \mathrm{E}_{i+1} & \text { if } x+y=3 \bmod 4\end{cases}
$$

whereas the remaining grey squares (those without coils in Figure 45) are described by (76), the usual $\check{R}$-matrix of the square lattice.

As a result we have an average of $\frac{3}{4}$ sites per grey square. While this might seem mediocre, it should be remembered that the $\check{R}$-matrix (78) for a conglomerate of two grey squares acts on six pairs of points, labelled $i, i+1, \ldots, i+5$ and $i^{\prime},(i+1)^{\prime}, \ldots,(i+5)^{\prime}$, which may in general accommodate Cat $(6)=132$ different connectivities. However, (78) contains only two terms, so that the number of connectivity states actually generated by the transfer process is only a very small subset of the total number of states respecting planarity. Accordingly we can attain a size of $n=16$, the largest for any of the problems studied in this article. Table 55 gives the corresponding results for the threshold $p_{\mathrm{c}}$. 


\begin{tabular}{r|l}
$n$ & $p_{\mathrm{c}}$ \\
\hline 4 & 0.6217033170149886495775240445635207543697869760484 \\
8 & 0.6218440760093613233443689965452296246219054789997 \\
12 & 0.6218163965094603058947746474408604618101513181220 \\
16 & 0.6218132249218005882705833731451247391116085233506 \\
\hline$\infty$ & $0.62181207(7)$ \\
Ref. [68] & $0.621819(3)$
\end{tabular}

Table 55. Site percolation threshold $p_{\mathrm{c}}$ on the ruby lattice.

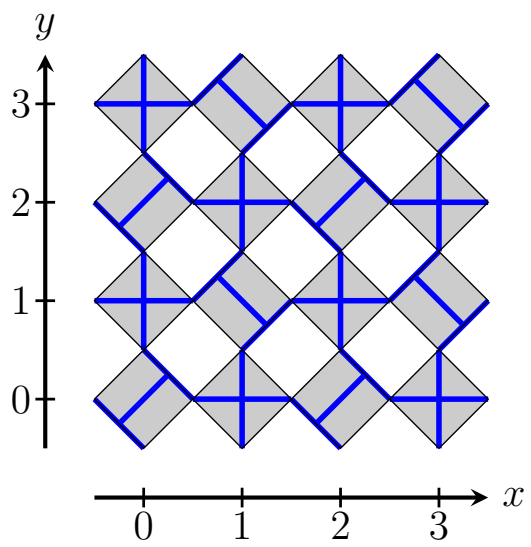

Figure 46. Four-terminal representation for site percolation on the Cairo pentagonal lattice.

\subsection{Cairo pentagonal lattice $D\left(3^{2}, 4,3,4\right)$}

Figure 46 provides a four-terminal representation of the Cairo pentagonal lattice. For the convenience of the drawing certain pairs of half-edges make an angle at the junction between neighboring grey squares, but such a pair should of course just be considered a single edge. The reader may verify that the lattice indeed consists of pentagons, and that going around each pentagon the degrees of the vertices are 3, 3, 4, 3 and 4 as they should be. There is on average $\frac{3}{2}$ vertices per grey square, and we must take $n$ even to respect the alternation of patterns.

The $\check{\mathrm{R}}$-matrix is given by that of the square lattice, Eq. (75), when $x+y$ is odd; by that of the hexagonal lattice, Eq. (73), when $x$ and $y$ are both even; and by a rotated version of the latter when $x$ and $y$ are both odd.

Results for the thresholds $p_{\mathrm{c}}$ are given in Table 56 .

\subsection{Frieze dual lattice $D\left(3^{3}, 4^{2}\right)$}

A four-terminal representation of the frieze dual lattice is shown in Figure 47. Its $\mathrm{R}_{\text {- }}$ matrix is that of the square lattice, Eq. (75), when $y$ is even; and that of the rotated hexagonal lattice, cf. Eq. (73), when $y$ is odd. All faces are pentagons, and the reader 


\begin{tabular}{l|l}
$n$ & $p_{\mathrm{c}}$ \\
\hline 2 & 0.6405124488065491504828379012985494181683890014987 \\
4 & 0.6500236759295320258852272011860502886365174620953 \\
6 & 0.6501636204301126095600239860376239822997457709415 \\
8 & 0.6501786803344847213746555063732068861224526222353 \\
\hline$\infty$ & $0.6501834(2)$ \\
Ref. [69] & $0.650184(5)$
\end{tabular}

Table 56. Site percolation threshold $p_{\mathrm{c}}$ on the Cairo pentagonal lattice.

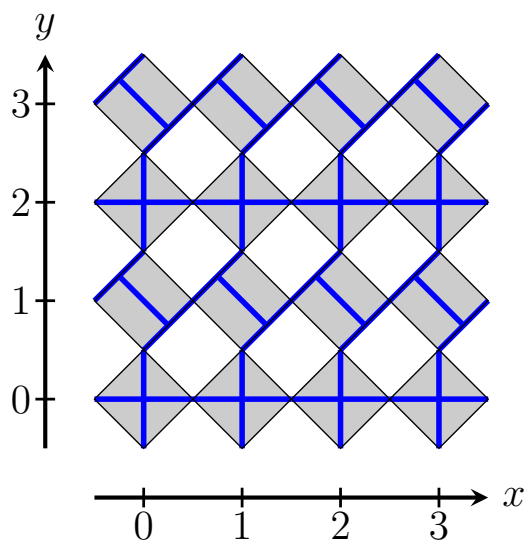

Figure 47. Four-terminal representation for site percolation on the frieze dual lattice.

may verify from the figure that the degrees of the surrounding vertices are $\left(3^{3}, 4^{2}\right)$ as they should be. There is on average $\frac{3}{2}$ vertices per grey square. Any parity of $n$ defines a valid basis, provided that we use $n \times 2 n$ rectangular bases in order to respect the alternation between rows.

The percolation thresholds $p_{\mathrm{c}}$ are displayed in Table 57.

\section{Discussion}

In this paper we have presented a new algorithm for the computation of the graph polynomial $P_{B}(q, v)$ associated with the $q$-state Potts model. This polynomial was introduced in [13] as a generalization of the bond percolation polynomial $P_{B}(1, v)$ studied in $[15,16,17]$. Just like properties of $P_{B}(1, v)$ were investigated for increasingly larger bases in $[18,19,20]$, we have here pursued the effort to compute $P_{B}(q, v)$ for the largest possible bases. This was achieved by means of a reformulation of the defining Eq. (6) within the periodic Temperley-Lieb algebra, and the use of powerful transfer matrix techniques. Thus, the computations in [13] for bases of size up to $|E|=36$ edges using the original deletion-contraction definition of $P_{B}(q, v)$-and that were improved to $|E|=144$ in [14] by means of a first, non-periodic transfer matrix approach (and even to $|E|=243$ using supercomputer facilities [14]) -were here carried to $|E|=882$ 


\begin{tabular}{l|l}
$n$ & $p_{\mathrm{c}}$ \\
\hline 1 & 0.6180339887498948482045868343656381177203091798058 \\
2 & 0.6378128305160144243967540410580253259072131959399 \\
3 & 0.6450649046998213833516533917200464266392184415175 \\
4 & 0.6463326652770031214369536045900433370913902143502 \\
5 & 0.6467492557573062279714940630727565301149079566625 \\
6 & 0.6469070727071462993287212489961496026254166173237 \\
7 & 0.6469742627385098566946369955909988391276996580357 \\
8 & 0.6470059040321607623572113208752443456723332267969 \\
9 & 0.6470222011016419470419039106312292679463616691412 \\
\hline$\infty$ & $0.6470471(2)$ \\
Ref. [69] & $0.647084(5)$
\end{tabular}

Table 57. Site percolation threshold $p_{\mathrm{c}}$ on the frieze dual lattice.

through the application of the novel transfer matrix formulation. Presumably these sizes can be pushed still farther by working out a parallelised version of the present algorithm and using supercomputer facilities [70].

These technical improvements have enabled us to refine the precision of the bond percolation thresholds $p_{\mathrm{c}}\left(\right.$ for $q=1$ ) and critical temperatures $v_{\mathrm{c}}$ (for general $q$ ) much beyond what was previously possible. For instance, from numerical simulations the values of $p_{\mathrm{c}}$ are known to a precision of the order $10^{-8}$ for the best-studied lattices. While the previous work on graph polynomials [14] yielded a comparable precision, this has now been carried to the order $10^{-13}$, which seems beyond the possibilities of Monte Carlo simulations for many years to come. This progress was made possible because of the extremely rapid convergence of the estimates coming from bases of finite size $n$, a fact that enabled us to extrapolate to the $n \rightarrow \infty$ limit using standard acceleration of convergence techniques.

It may appear surprising at first sight that the use of extrapolation techniques may add, in the most favourable cases, 2 or even 3 extra digits of precision to those that appear to have converged from a mere visual inspection of the last two finite- $n$ results. We stress that this kind of accuracy relies both on the rapid and well-behaved convergence - and in particular on the high value of $w$ in (30) - and on the fact that our finite- $n$ data are exact results, i.e., without error bars. The tables in this paper report these results with 50-digit numerical precision, enabling sceptical readers to check our final results with their own extrapolation procedures.

The comments about the precision of the results also apply to the values of $v_{\mathrm{c}}$ for general $q$, in particular for the notably tricky case of $q=4$ where numerical simulations of the Monte Carlo or transfer matrix type are hampered by strong logarithmic corrections to scaling, whilst the graph polynomial method seems to encounter no noticeable loss of precision. 
To illustrate the versatility of the graph polynomial method we have also extended the study of the most common lattices [14] to a considerably larger set consisting of all Archimedean lattices, their duals, and their medials. For some of these lattices parity constraints on $n$ applied, implying fewer data points and hence less precise extrapolations. It nevertheless remains true that we have been able to improve - often significantly - on the precision of the bond percolation thresholds for essentially all of the lattices studied here, and that have previously been addressed using other methods (see [65] for a review). Other lattices, notably some of the medial lattices, have to our knowledge never been investigated before.

The $q$-state Potts model appears to have been studied only on a more restricted class of lattices than percolation. Therefore, many of our results for the Potts model are new and cannot be compared to existing work.

An important feature of the graph polynomial $P_{B}(q, v)$ is that its factorisation appears to signal cases of exact solvability $[13,14]$. This is nowhere more conspicuous than for the Ising model $(q=2)$, for which our factorised results successfully reproduce the known critical temperatures $v_{\mathrm{c}}$ for all of the Archimedean lattices [23]. We have presented this type of results also for all the medial lattices, which do not appear to have been much studied before.

More generally, in sections 4.11 and 6.8 we have systematically searched for cases where $P_{B}(q, v)$ factorises. We have thus identified many cases of presumed exact solvability, including models of spanning forests, chromatic and flow polynomials, and Potts models with integer values of $q$. Each of these cases deserves a specific study, opening possibilities for much future work. We should also point out that the threestate antiferromagnet, $(q, v)=(3,-1)$, appears to play a special role for many of the lattices studied: even when the curves $P_{B}(q, v)=0$ do not pass though this point exactly, they often come increasingly close upon increasing the size $n$.

Apart from producing extremely precise numerical values for $p_{\mathrm{c}}$ and $v_{\mathrm{c}}$, the graph polynomials have also emerged as a powerful tool for studying the full phase diagram of the Potts model in the real $(q, v)$ plane. While this was realised already in the first study of the kagome lattice [13], and increasingly so with the extension to some of the other most well-known lattices [14], we have here seen many new features emerge as results of the improved precision and the greater variety of lattices being studied. In particular, the presence of phase transitions at the Beraha numbers, $q=B_{k}$ with even index $k=4,6,8, \ldots$ in $(21)$, has been firmly established for all the lattices. These transitions take place inside the Berker-Kadanoff phase [39] whose extent can thus be inferred from the size and positions of the vertical rays at the Beraha numbers.

Beyond this, the phase diagram inside the antiferromagnetic region $v<0$ turns out to be dauntingly complicated and highly lattice dependent. Often extra curves exist outside the BK phase, or cutting through it, and the question whether these curves are critical and, if so, what is the precise nature of the criticality, remains in most cases open. In particular, numerical transfer matrix studies would be a precious help to assess whether the parts of these curves with $q \in[0,4]$ enjoy conformal invariance. 
A first such study was made for the kagome lattice in [13, Figure 12], but clearly much more work is needed. The cases where extra curves are present inside the BK phase are particularly interesting, since then presumably the relevant critical theory consists of conformal excitations on top of a highly excited level inside the BK phase that could only be accessed by changing from the loop (or FK cluster) representation to another (e.g., RSOS height) representation of the Temperley-Lieb algebra. Another intriguing feature is the possibility of the critical curves to be space filling, the strongest evidence for which is provided by the frieze medial lattice (see in particular Figure 30). This is reminiscent of the "critical regions" found in recent transfer matrix studies of the $q$-state Potts model [66], including on planar lattices [47]. Finally we note that most of the phase diagrams tend to become increasingly complicated as $q \rightarrow 4$ (see Figure 16 for a vivid illustration), and that for $q>4$ - a regime which we have chosen not to discuss in the present paper - the curves again appear to develop a space filling behaviour for several lattices. This should again be compared with the outcome of other studies of the critical behaviour of antiferromagnetic Potts models close to [45, 46], at [71] and beyond $[66,72] q=4$. Obviously, a detailed study of all these aspects for at least one of the lattices presented here would add much substance to these observations [47].

On a more technical level, we have shown how to write all the lattices under investigation in the four-terminal representation of Figure 2. In many cases, these representations are not at all obvious to come by (see section 4.7 and Figure 17 for an example). The importance of such representations transcends the applications made in the present paper. In particular, these representations provide, for each lattice, an efficient sparse-matrix factorised construction of the transfer matrix that can be immediately applied in numerical studies. It forms part of the interest of studying so many different lattices, that we have discovered several tricks by which the basic four-terminal representation can be adapted to new situations. These tricks include the addition of horizontal diagonals on the white squares (section 4.1), their extension to "white hexagonals" with even more structure (section 4.9), the "generic Ř-matrix algorithm" that avoids having to add extra auxiliary points inside the grey squares (section 6.1), the use of contracted edges (section 6.4), and the possibility of having correlated vertices (section 7.5). Some of these improvements of the general method have turned out particularly useful in order to be able to access also site percolation on a variety of lattices.

We finally comment on the values of the parameter $w$ appearing in the finite-size scaling form (30). For simplicity we focus here on the case of (bond or site) percolation on the lattices for which we have a sufficiently (at least seven) large number of sizes $n$ to allow for a precise determination of the effective values of $w$ (the numbers quoted are based on the three largest sizes). We have seen that $w$ takes essentially the same values for bond percolation on the kagome $(w \approx 6.36)$, the three-twelve $(w \approx 6.39)$, and the three-twelve medial lattice $(w \approx 6.38)$, as well as for site percolation on the hexagonal lattice $(w \approx 6.35)$. It is remarkable that all those lattices have a three-fold rotational symmetry. Another value of $w$ is taken for bond percolation on the four-eight 
( $w \approx 4.28)$, the four-eight medial $(w \approx 4.29)$, and the frieze medial lattice $(w \approx 4.59)$, as well as for site percolation on the square $(w \approx 4.07)$, the four-eight $(w \approx 4.40)$, and the frieze dual lattice $(w \approx 4.25)$. Although there is a larger spread in those values, it is tempting to speculate that they might in fact be finite-size approximations to a common value applying to the lattices with a four-fold rotational symmetry. We stress that it is the value for site percolation on the square lattice, $w \approx 4.07$, that is determined with the largest reliability, since it is based on eleven sizes $n$ (see section 7.2). It is conceivable that the exact value might be $4+\frac{5}{48} \simeq 4.104 \cdots$, where $2 h=\frac{5}{48}$ is the critical exponent giving the co-dimension of a bulk percolation cluster. This corresponds in the transfer matrix formalism [73] to setting the weight of winding loops $n_{\text {wind }}=0$, which, as we have seen in section 3.5.2, is the proper condition to discard contributions from $Z_{1 \mathrm{D}}$ in (6). If the value of $w$, and more generally of further correction-to-scaling exponents, could be determined exactly one should be able to produce even more precise extrapolations $p_{\mathrm{c}}$ from the existing data. $\dagger$ We leave this important question for future work.

\section{Acknowledgments}

The author's work was supported by the Agence Nationale de la Recherche (grant ANR10-BLAN-0414: DIME) and the Institut Universitaire de France. He warmly thanks

C. R. Scullard for collaboration on related projects, and for many helpful comments and encouragement throughout the process of writing up this long paper. The author would like to express his gratitude for the hospitality of the Galileo Galilei Institute for Theoretical Physics, Florence, where a crucial part of this work was carried out (in April 2012). He also thanks A.J. Guttmann for discussions about extrapolation methods. The numerical computations reported here were performed at the High Performance Computing center at New York University and made possible by a generous donation from the Dell Corporation and the kind permission of A.D. Sokal.

\section{References}

[1] Onsager L 1944 Phys. Rev. 65 117-149

[2] Kasteleyn P W 1961 Physica 27 1209-1225

[3] Lieb E H 1967 Phys. Rev. 162 162-172

[4] Baxter R J 1972 Ann. Phys. 70 193-228

[5] Baxter R J 1973 J. Phys. C 6 L445

[6] Potts R B 1952 Proc. Camb. Phil. Soc. 48106

[7] Fortuin C M and Kasteleyn P W 1972 Physica 57536

[8] Baxter R J, Temperley H N V and Ashley S E 1978 Proc. Roy. Soc. London A 358535

[9] Scullard C R and Jacobsen J L 2013 J. Phys. A: Math. Theor. 46125006

[10] Wu F Y 2006 Phys. Rev. Lett. 9690602

[11] Wu F Y and Lin K Y 1980 J. Phys. A 13 629-636

[12] Wu F Y 1979 J. Phys. C 12 L645

$\dagger$ See [74] for an example of what gain in precision in the determination of the critical point can be obtained from a thorough understanding of the finite-size scaling exponents. 
[13] Jacobsen J L and Scullard C R 2012 J. Phys. A: Math. Theor. 45494003

[14] Jacobsen J L and Scullard C R 2013 J. Phys. A: Math. Theor. 46075001

[15] Scullard C R and Ziff R M 2006 Phys. Rev. E 73 045102(R)

[16] Scullard C R and Ziff R M 2008 Phys. Rev. Lett. 100185701

[17] Scullard C R and Ziff R M 2010 J. Stat. Mech. 2010 P03021

[18] Scullard C R 2011 J. Stat. Mech. 2011 P09022

[19] Scullard C R 2012 Phys. Rev. E 86041131

[20] Scullard C R 2012 J. Stat. Mech.: Theor. Exp. 2012 P11011

[21] Scullard C R and Jacobsen J L 2012 J. Phys. A: Math. Theor. 45494004

[22] Ding C, Fu Z, Guo W and Wu F Y 2010 Phys. Rev. E 81061111

[23] Codello A 2010 J. Phys. A: Math. Theor. 43385002

[24] Jensen I, Gutmann A J and Enting I G 1997 J. Phys. A: Math. Gen. 30 8067-8083

[25] Feng X, Deng Y and Blöte H W J 2008 Phys. Rev. E 78031136

[26] Grünbaum B and Shephard G C 1987 Tilings and Patterns (Freeman, New York)

[27] Wu F Y and Wang Y K 1976 J. Math. Phys. 17 439-440

[28] Baxter R J, Kelland S B and Wu F Y 1976 J. Phys. A: Math. Gen. 9397

[29] Essam J W and Fisher M E 1961 J. Math. Phys. 2609

[30] Sykes M F and Essam J W 1964 J. Math. Phys. 51117

[31] Temperley H N V and Lieb E H 1971 Proc. R. Soc. London A 322251

[32] Vasseur R, Gainutdinov A, Jacobsen J L and Saleur H 2014 in preparation

[33] Jones V F R 1994 L'Enseignement Math. 40313

[34] Blöre H W J and Nightingale M P 1982 Physica A 112 405-465

[35] Blöre H W J and Nienhuis B 1989 J. Phys. A: Math. Gen. 221415

[36] Haible B CLN - Class library for numbers http://www.ginac.de/CLN/

[37] Baxter R J, Temperley H N V and Ashley S E 1978 Proc. R. Soc. London A 358535

[38] Baxter R J 1982 Proc. Roy. Soc. London A 38343

[39] Saleur H 1991 Nucl. Phys. B 360219

[40] Jacobsen J L, Salas J and Sokal A D 2003 J. Stat. Phys. 112 921-1017

[41] Jacobsen J L and Salas J 2006 J. Stat. Phys. 122705

[42] Jacobsen J L and Salas J 2007 Nucl. Phys. B $\mathbf{7 8 3} 238$

[43] Jacobsen J L and Saleur H 2006 Nucl. Phys. B 743207

[44] Nienhuis B 1982 Phys. Rev. Lett. 49 1062-1065

[45] Baxter R J 1986 J. Phys. A: Math. Gen. 192821

[46] Baxter R J 1987 J. Phys. A: Math. Gen. 205241

[47] Jacobsen J L, Salas J and Scullard C R 2014 in preparation

[48] Ikhlef Y, Jacobsen J L and Saleur H 2008 Nucl. Phys. B 789 483-524

[49] Ikhlef Y, Jacobsen J L and Saleur H 2012 Phys. Rev. Lett. 108081601

[50] Bulirsch R and Stoer J 1964 Numer. Math. 6 413-427

[51] Monroe J L 2002 Phys. Rev. E 65066116

[52] Kano K and Naya S 1953 Prog. Theor. Phys. 10158

[53] Moore C and Newman M E J 2000 J. Stat. Phys. 99 629-660

[54] Parviainen R 2007 J. Phys. A: Math. Gen. 409253

[55] Utiyama T 1951 Prog. Theor. Phys. 6907

[56] Syozi I 1955 Rev. Kobe Univ. Mercantile Marine 221

[57] Syozi I 1972 Transformation of Ising models Phase transitions and critical phenomena vol 1 ed Domb C and Green M S (New York: Wiley) p 269

[58] Codello A 2010 J. Phys. A: Math. Theor. 43399801

[59] Wu F Y 1988 J. Stat. Phys. 52 99-112

[60] Jacobsen J L and Salas J 2013 J. Combin. Theory B 103 532-565

[61] Jacobsen J L, Salas J and Sokal A 2005 J. Stat. Phys. 1191153

[62] Caracciolo S, Jacobsen J L, Saleur H, Sokal A D and Sportiello A 2004 Phys. Rev. Lett. 93080601 
[63] Jacobsen J L and Saleur H 2005 Nucl. Phys. B 716 439-461

[64] Ziff R M unpublished

[65] Ziff R M 2014 Wikipedia entry "Percolation threshold"

[66] Jacobsen J L and Salas J 2013 Nucl. Phys. B 875 678-718

[67] Kunz H and Wu F Y 1978 J. Phys. C: Solid State Phys. 11 L1

[68] Suding P N and Ziff R M 1999 Phys. Rev. E 60275

[69] Parviainen R 2004 Connectivity properties of Archimedean and Laves lattices Ph.D. thesis Uppsala University

[70] Jacobsen J L and Scullard C R 2014 in preparation

[71] Deng Y, Huang Y, Jacobsen J L, Salas J and Sokal A 2011 Phys. Rev. Lett. 107150601

[72] Huang Y, Chen K, Deng Y, Jacobsen J L, Kotecký R, Salas J, Sokal A D and Swart J M 2013 Phys. Rev. E $\mathbf{8 7} 012136$

[73] Jacobsen J L 2009 Conformal field theory applied to loop models, in Polygons, polyominoes and polycubes, Lecture Notes in Physics vol $\mathbf{7 7 5}$ ed Guttmann A J (Heidelberg: Springer Verlag) pp $347-424$

[74] Guo W and Blöte H W J 2002 Phys. Rev. E 66046140 